\author{
UNIVERSIDADE DE SÃO PAULO \\ ESCOLA DE ENGENHARIA DE SÃO CARLOS \\ DEPARTAMENTO DE ENGENHARIA DE ESTRUTURAS
}

JEFERSON WILIAN DOSSA FERNANDES

Interação Fluido-Estrutura com escoamentos incompressíveis utilizando o Método dos Elementos Finitos 

JEFERSON WILIAN DOSSA FERNANDES

\title{
Interação Fluido-Estrutura com escoamentos incompressíveis utilizando o Método dos Elementos Finitos
}

\author{
VERSÃO CORRIGIDA \\ A versão original encontra-se na Escola de Engenharia de São Carlos
}

Dissertação apresentada ao Departamento de Engenharia de Estruturas da Escola de Engenharia de São Carlos, Universidade de São Paulo, como parte dos quesitos necessários para a obtenção do título de Mestre em Engenharia Civil (Estruturas).

Orientador: Prof. Dr. Rodolfo André Kuche Sanches 
AUTORIZO A REPRODUÇÃO TOTAL OU PARCIAL DESTE TRABALHO, POR QUALQUER MEIO CONVENCIONAL OU ELETRÔNICO, PARA FINS DE ESTUDO E PESQUISA, DESDE QUE CITADA A FONTE.

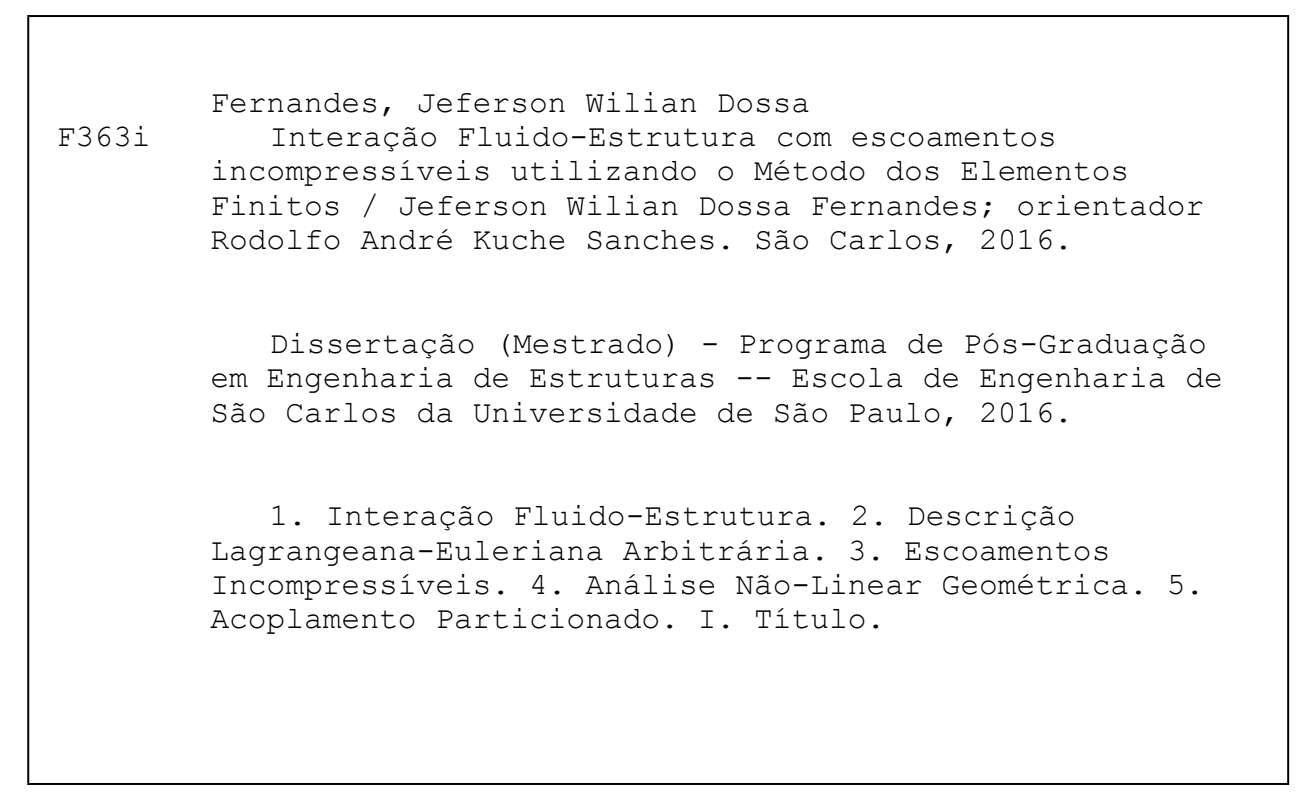




\section{FOLHA DE JULGAMENTO}

Candidato: Engenheiro JEFERSON WILIAN DOSSA FERNANDES.

Título da dissertação: "Interação fluido-estrutura com escoamento incompressíveis utilizando o método dos elementos finitos".

Data da defesa: 01/03/2016

\section{Comissão Julgadora:}

Prof. Dr. Rodolfo André Kuche Sanches (Orientador)

(Escola de Engenharia de São Carlos/EESC)
Resultado:

Aprovado

Prof. Associado Fabrício Simeoni de Sousa

APROVADO

(Instituto de Ciências Matemáticas e de Computação/ICMC)

Prof. Dr. Marcos Arndt

(Universidade Federal do Paraná/UFPR)

Aprovado

Coordenador do Programa de Pós-Graduação em Engenheira Civil (Engenharia de Estruturas):

Prof. Titular Humberto Breves Coda

Presidente da Comissão de Pós-Graduação:

Prof. Associado Paulo Sergio Lima Segantine 

Para minha família, em especial à Vera, Zaqueu, Laryssa, Lucas, Marina (in memoriam) e Lindolfina. 

"É preciso que eu suporte duas ou três lagartas, se eu quiser conhecer as borboletas."

(Antoine de Saint-Exupéry, trecho de "O Pequeno Príncipe") 

Primeiramente à minha família, em especial aos meus pais e irmãos pela motivação, por acreditarem em mim e nunca me deixarem desistir.

Meus sinceros agradecimentos ao professor Dr. Rodolfo André Kuche Sanches, não somente pela orientação e dedicação depositados a mim e a este trabalho, mas também por ter desempenhado papel singular em minha vida acadêmica.

Aos companheiros de luta do SET, sem os quais tudo seria menos divertido.

Ao Departamento de Engenharia de Estruturas, à Escola de Engenharia de São Carlos e à Universidade de São Paulo, pela infraestrutura disponibilizada para o desenvolvimento desta pesquisa.

Aos professores Tit. Humberto Breves Coda e Dr. Rodrigo Ribeiro Paccola pelas contribuições na banca de qualificação e também pela disponibilização e ajuda com o programa de casca dinâmica não linear geométrica.

Aos demais professores que tive a oportunidade de conviver durante o período de mestrado, sempre dispostos a auxiliar e transmitir conhecimento.

Por fim, à Coordenação de Aperfeiçoamento de Pessoal de Nível Superior (CAPES) pelo suporte financeiro. 



\section{RESUMO}

\section{FERNANDES, J. W. D. Interação Fluido-Estrutura com escoamentos incompressíveis}

utilizando o Método dos Elementos Finitos. 2016. 120 p. Dissertação (Mestrado em

Engenharia de Estruturas) - Departamento de Engenharia de Estruturas, Escola de Engenharia de São Carlos, Universidade de São Paulo, São Carlos, 2016.

A interação entre fluidos e estruturas caracteriza um problema multi-físico não linear e está presente numa grande variedade de áreas da engenharia. Este trabalho apresenta o desenvolvimento de ferramentas computacionais com base no Método dos Elementos Finitos (MEF) para a análise de interação fluido-estrutura (IFE) considerando escoamentos com baixas velocidades. Dada a interdisciplinaridade do tema, se faz necessário o estudo em três diferentes assuntos: a dinâmica das estruturas computacional, a dinâmica dos fluidos computacional, e o problema de acoplamento. No caso da dinâmica das estruturas empregar-se um elemento finito que seja adequado para a simulação de problemas de IFE, que claramente demandam uma análise não linear geométrica, optando-se pelo emprego de uma formulação descrita em posições, a qual evita problemas relativos à aproximação de rotações finitas. Quanto à dinâmica dos fluidos computacional, é empregado um método estável e ao mesmo tempo sensível à movimentação da estrutura, utilizando a descrição Lagrangeana-Euleriana Arbitrária (ALE). Os casos considerados neste trabalho, assim como muitos dos problemas de engenharia, ocorrem com escoamentos em baixas velocidades, implicando na incompressibilidade do fluido, o que demanda, para um método estável, a utilização de elementos que atendam à condição de Ladyzhenskaya-BabuškaBrezzi (LBB). Além disso, é necessário também o emprego de métodos que consigam neutralizar as variações espúrias decorrentes da não-linearidade de possíveis escoamentos com convecção dominante e que surgem com a aplicação do processo clássico de Galerkin. Para superar esse problema, é aplicado o método Streamline-Upwind/Petrov-Galerkin (SUPG), que adiciona difusividade artificial na direção do escoamento, controlando a amplitude dos termos convectivos. No que se refere ao acoplamento fluido-casca, buscam-se modularidade e versatilidade adotandose o modelo particionado. O modelo de acoplamento implementado garante ainda a utilização de malhas do fluido e da estrutura sem a necessidade de coincidência de nós.

Palavras-chave: Interação Fluido-Estrutura. Descrição Lagrangeana-Euleriana Arbitrária. Escoamentos Incompressíveis. Análise Não-Linear Geométrica. Acoplamento Particionado. 



\section{ABSTRACT}

\section{FERNANDES, J. W. D. Incompressible Fluid-Structure Interaction using the Finite}

Element Method. 2016. 120 p. Dissertation (M. Sc. In Structural Engineering) - Department of Structural Engineering, School of Engineering of São Carlos, University of São Paulo, São Carlos, 2016.

Interaction between fluids and structures characterizes a nonlinear multi-physics problem present in a wide range of engineering fields. This works presets the development of computational tools based on finite element method (FEM) for fluid-structure interaction (FSI) analysis considering low speed flows (incompressible), as a great part of the engineering problems. Given the topic multidisciplinary nature, it is necessary to study three different subjects: the computational structural dynamics, the computational fluid mechanics and the coupling problem. Regarding structural mechanics, we seek to employ a finite element adequate to FSI simulation, what clearly demands a geometric nonlinear analysis. We chose to employ shell elements with formulation in terms of positions, which avoids problems related to finite rotations approximations. Concerning computational fluid dynamics, we employ a stable method, at same time sensible o structural movements, which is written in the arbitrary Lagrangian-Eulerian (ALE) description. The flow incompressibility demands, for a stable method, the use of elements according to the Ladyzhenskaya-Bbuska-Brezzi (LBB) condition. It is also necessary to employ methods able to neutralize the spurious variations that appears from convection dominated flows when applying the standard Galerking method. In order to overcome this problem, we apply the Streamline-Upwind/Petrov-Galerkin (SUPG) method, which adds artificial diffusivity to the streamline direction, controlling spurious variations. Considering the fluid-shell coupling, we seek modularity and versatility, adopting the partitioned model. The developed coupling model ensure the use of fluid and structure meshes with no need for matching nodes.

Key-words: Fluid-Structure Interaction. Arbitrary Lagrangian-Eulerian Description. Incompressible Flows. Geometric Nonlinear Analysis. Partitioned Coupling. 



\section{LISTA DE ILUSTRAÇÕES}

Figura 1.1 - Ponte de Tacoma Narrows (a) instantes antes do colapso e (b) após a ruína. . 26 Figura 1.2 - Diagrama de Collar . . . . . . . . . . . . . . . . . . 27

Figura 1.3 - Buffeting ou martelamento . . . . . . . . . . . . . . . . . . . 29

Figura 1.4 - Esquema estrutural simplificado de uma asa de avião . . . . . . . . . . . . 29

Figura 2.1 - Volume de controle infinitesimal e balanço do fluxo de massa . . . . . . . . 40

Figura 2.2 - Volume de controle infinitesimal e balanço do fluxo da quantidade de movimento 42

Figura 2.3 - Cinemática da descrição ALE. . . . . . . . . . . . . . . . . . . . . 45

Figura 3.1 - Cinemática de um sólido deformável. . . . . . . . . . . . . . . . . . . . . . 48

Figura 3.2 - Elemento de barra sendo tracionado. . . . . . . . . . . . . . . . . . . 50

Figura 4.1 - Elementos finitos que atendem à condição LBB . . . . . . . . . . . . . . . . 56

Figura 4.2 - Elemento tetraédrico isoparamétrico de aproximação linear para pressão e quadrática para velocidade. . . . . . . . . . . . . . . . . . 57

Figura 4.3 - Fluxograma do procedimento de avanço temporal da solução. . . . . . . . . 62

Figura 4.4 - Geometria e condições de contorno do problema da cavidade quadrada. . . . 63

Figura 4.5 - Malhas de elementos finitos utilizadas: (a) malha 1, (b) malha 2 e (c) malha 3.64

Figura 4.6 - Velocidades adimensionais em função da altura/largura da cavidade. . . . . . 65

Figura 4.7 - Linhas de corrente obtidas por Glowinski (2003) (à esquerda) e no presente trabalho.

Figura 4.7 - Linhas de corrente obtidas por Glowinski (2003) (à esquerda) e no presente trabalho - continuação.

Figura 4.8 - Linhas de pressão obtidas por Glowinski (2003) (à esquerda) e no presente trabalho.

Figura 4.8 - Linhas de pressão obtidas por Glowinski (2003) (à esquerda) e no presente trabalho - continuação. . . . . . . . . . . . . . . . 68

Figura 4.9 - Geometria do problema da barragem rompida. . . . . . . . . . . . . . . 69

Figura 4.10-Malha utilizada na simulação do problema da barragem rompida. . . . . . . 69

Figura 4.11-Deslocamento horizontal relativo em função do tempo adimensional. . . . . . 70

Figura 4.12-Instante de tempo t* $=1,5$ : (a) malha deformada, (b) pressão, (c) velocidade $x \mathrm{e}(\mathrm{d})$ velocidade $y \ldots \ldots \ldots \ldots \ldots$

Figura 4.13-Instante de tempo $t^{*}=2,5$ : (a) malha deformada, (b) pressão, (c) velocidade $x$ e (d) velocidade $y \ldots \ldots \ldots \ldots$. . . . . . . . . . . . . . . . .

Figura 4.14-Instante de tempo $t^{*}=4,0$ : (a) malha deformada, (b) pressão, (c) velocidade $x$ e (d) velocidade $y$. 
Figura 4.15-Geometria do problema de escoamento em torno de um cilindro. . . . . . . . 74

Figura 4.16-Malha utilizada nas simulações de escoamento em torno de um cilindro. . . 74

Figura 4.17-Componente vertical de velocidade no ponto A ao longo do tempo. . . . . . 75

Figura 4.18-Coeficiente de (a) arrasto e (b) sustentação ao longo do tempo para $\Delta t=0,1 . \quad 76$

Figura 4.19-Coeficiente de (a) arrasto e (b) sustentação ao longo do tempo para $\Delta t=0,05.76$

Figura 4.20-Linhas de pressão em t=100,0 (a) Najafi, Arefmanesh e Enjilela (2012) e (b)

Presente trabalho. . . . . . . . . . . . . . . . . 77

Figura 4.21-Linhas de corrente em $t=100,0$ (a) Najafi, Arefmanesh e Enjilela (2012) e (b)

Presente trabalho. . . . . . . . . . . . . . . . . 78

Figura 5.1 - Cinemática do MEF Posicional. . . . . . . . . . . . . . . . . . . . . . 80

Figura 5.2 - Cinemática do elemento finito de casca. . . . . . . . . . . . . . . 82

Figura 5.3 - Fluxograma do procedimento de avanço temporal da solução. . . . . . . . . 86

Figura 5.4 - Geometria e condições de contorno da viga engastada com carregamento distribuído uniforme. . . . . . . . . . . . . . . . . . 86

Figura 5.5 - Malha de elementos finitos utilizada para a discretização da viga. . . . . . . 87

Figura 5.6 - Deslocamento vertical da extremidade da viga em função do tempo. . . . 87

Figura 5.7 - Configurações deformadas da viga engastada em diferentes instantes de tempo. 88

Figura 5.8 - Geometria, discretização e comportamento temporal do carregamento para o exemplo de snap-through dinâmico. . . . . . . . . . . . . . . . . . . . . . 89

Figura 5.9 - Deslocamento vertical no centro da casca em função do tempo. . . . . . . . 89

Figura 5.10-Resultados obtidos por Argyris, Papadrakakis e Mouroutis (2003) para o problema da casca cilíndrica com snap-through dinâmico. . . . . . . . . . . 90

Figura 5.11-Configurações deformadas da casca para diferentes instantes de tempo. . . . 90

Figura 6.1 - Representação esquemática do domínio computacional $\Omega$. . . . . . . . . . 91

Figura 6.2 - Nós pertencentes à interface $\Gamma_{f s} \ldots \ldots \ldots$. . . . . . . . . . . 93

Figura 6.3 - Representação da discretização do fluido imersa na malha auxiliar . . . . . 94

Figura 6.4 - Esquema de acoplamento particionado fraco . . . . . . . . . . . . . . . 97

Figura 6.5 - Geometria e condições de contorno do problema da cavidade com fundo flexível. . . . . . . . . . . . . . . . . . . . . 98

Figura 6.6 - Malha auxiliar para movimentação do domínio do fluido. . . . . . . . . . . 98

Figura 6.7 - Deslocamento vertical do ponto A em função do tempo. . . . . . . . . . . . 99

Figura 6.8 - Distribuição de pressões e linhas de corrente para diversos instantes de tempo. 99

Figura 6.8 - Distribuição de pressões e linhas de corrente para diversos instantes de tempo - continuação. . . . . . . . . . . . . . . . . 100

Figura 6.9 - Configurações deformadas da malha 3 e da malha auxiliar para diversos instantes de tempo. . . . . . . . . . . . . . . . . . . 100

Figura 6.10-Geometria e condições de contorno do problema da cavidade 3D. . . . . . . 101

Figura $6.11-$ Discretização da estrutura. . . . . . . . . . . . . . . . . . . . . . 102

Figura 6.12-Geometria e condições de contorno do problema da cavidade 3D. . . . . . . 102 
Figura 6.13-Deslocamento vertical do ponto B em função do tempo. . . . . . . . . . . . . 103

Figura 6.14-Configurações deformadas da base flexível em vários instantes de tempo. . . 103

Figura 6.15-Vista da distribuição de pressões na seção central da cavidade. . . . . . . . 103

Figura 6.16-Vista da distribuição de velocidades na seção central da cavidade. . . . . . . 104

Figura 6.17-Geometria e condições de contorno do problema do painel flexível. . . . . . 104

Figura 6.18-Vista frontal das malhas de elementos finitos empregadas na análise do problema do painel flexível. . . . . . . . . . . . . . . . 105

Figura 6.19-Deslocamento vertical do ponto $\mathrm{C}$ em função do tempo. . . . . . . . . . . . 106

Figura 6.20-Distribuição da componente $X$ de velocidade em diferentes instantes de tempo.107

Figura 6.21-Distribuiçãa de pressão em diferentes instantes de tempo. . . . . . . . . . . 108

Figura 6.22-Configuração deformada da malha do fluido na região da interface com a estrutura em diferentes instantes de tempo. . . . . . . . . . . . . . . . . . . 109 

Tabela 1.1 - Classificação dos problemas aeroelásticos . . . . . . . . . . . . 28

Tabela 4.1 - Características das malhas utilizadas no problema da cavidade quadrada . . 64

Tabela 4.2 - Comparativo entre os valores de $C_{D, \text { médio }}, C_{L, \text { máximo }}$ e St. . . . . . . . . . 77 



\section{LISTA DE SÍMBOLOS}

Os significados dos símbolos estão descritos à medida em que aparecem no texto.

Foram utilizadas as notações indicial e diádica, quando convenientes, de modo que símbolos em negrito representam vetores, matrizes ou tensores. 

INTRODUÇÃ̃O . . . . . . . . . . . . . . . . . . 25

1.1 Conceituação do problema de interação fluido-estrutura . . . . . . . . . 27

1.1.1 Problemas estáticos de resposta . . . . . . . . . . . . . . . . . . 28

1.1.2 Problemas dinâmicos de resposta . . . . . . . . . . . . . . . . . . . . 28

1.1.3 Problemas estáticos de estabilidade . . . . . . . . . . . . . . . . . . 29

1.1.4 Problemas dinâmicos de estabilidade . . . . . . . . . . . . . . . . . . . 29

$1.2 \quad$ Estado da arte . . . . . . . . . . . . . . . . . . 30

1.2.1 Dinâmica dos Fluidos Computacional . . . . . . . . . . . . . . . . 30

1.2.2 Dinâmica das Estruturas Computacional . . . . . . . . . . . . . . . . 32

1.2.3 Problema de Acoplamento . . . . . . . . . . . . . . . . . 34

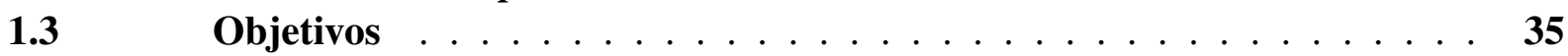

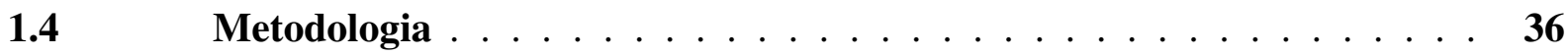

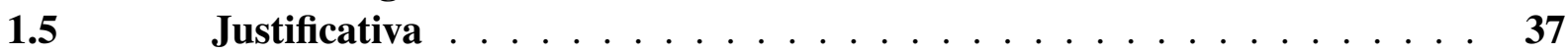

2 MECÂNICA DOS FLUIDOS INCOMPRESSÍVEIS . . . . . . . 39

$2.1 \quad$ Conservação da massa $\ldots \ldots \ldots \ldots \ldots$

2.2 Conservação da quantidade de movimento . . . . . . . . . . . . . . . 41

2.3 Conservação da energia . . . . . . . . . . . . . . . . 43

2.4 Definição do problema incompressível isotérmico . . . . . . . . . . 43

2.5 Equações governantes na descrição Lagrangeana-Euleriana Arbitrária (ALE) . . . . . . . . . . . . . . . . . . . . 44

MECÂNICA DOS SÓLIDOS . . . . . . . . . . . . . 47

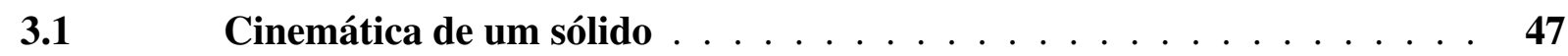

$3.2 \quad$ Equilíbrio de um sólido $\ldots \ldots \ldots \ldots \ldots \ldots$

$3.3 \quad$ Medida de deformação e lei constitutiva $\ldots \ldots \ldots$. . . . . . . . . . . 49

4 ANÁLISE NUMÉRICA DOS ESCOAMENTOS INCOMPRESSÍVEIS . 53

$4.1 \quad$ Forma fraca e aproximação pelo MEF $\ldots \ldots \ldots \ldots \ldots$

$4.2 \quad$ Estabilidade da formulação $\ldots \ldots \ldots \ldots \ldots \ldots$

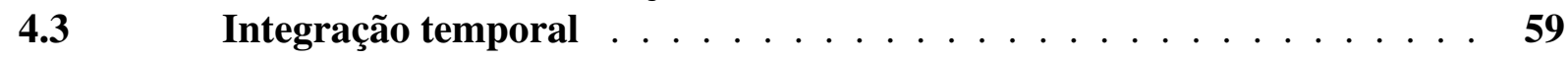

4.3.1 Integrador de primeira ordem . . . . . . . . . . . . . . 59

4.3.2 Integrador de segunda ordem . . . . . . . . . . . . . . . . . 61

4.3.3 Procedimento para o avanço temporal da solução . . . . . . . . . . . . . 62

$4.4 \quad$ Exemplos de problemas de dinâmica dos fluidos . . . . . . . . . . . 62

4.4.1 Cavidade quadrada . . . . . . . . . . . . . . . . 63

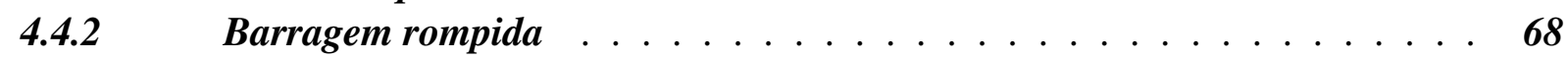

4.4.3 Escoamento em torno de um cilindro . . . . . . . . . . . . . . . . . . . . 74

5 ANÁLISE NUMÉRICA DA MECÂNICA DOS SÓLIDOS . . . . . . 79

$5.1 \quad$ A cinemática do MEF Posicional . . . . . . . . . . . . . . 79

$5.2 \quad$ O MEF Posicional aplicado ao elemento finito de casca . . . . . . . . . . 81 
5.5.1 Viga engastada com carregamento distribuído uniforme . . . . . . . . . . 86

$\quad$ 5.5.2 Casca cilíndrica com Snap-through dinâmico . . . . . . . . . . . . . 88

ACOPLAMENTO FLUIDO-CASCA . . . . . . . . . . . . . . 91

Condições de acoplamento - transferência de forças e velocidades . . . . 91 Painel flexível sob a ação de vórtices gerados por um prisma de seção quadrada . . . . . . . . . . . . . . . . . . . 104 
Problemas de interação fluido-estrutura (IFE) são muito comuns, se manifestando nas mais diversas áreas da engenharia. Na engenharia civil, por exemplo, tem-se a ação do vento sobre estruturas, escoamentos em canais e vertedores, a água interagindo com barragens e estruturas off-shore dentre outros. Sua presença estende-se ainda à engenharia aeronáutica, hidráulica e até mesmo à bioengenharia na simulação de escoamentos cardiovasculares, por exemplo. Em todos os casos supracitados é possível observar escoamentos com baixo número de Mach (razão entre a velocidade característica do escoamento e a velocidade do som no fluido), isto é, podendo ser considerados incompressíveis.

Os escoamentos incompressíveis são assim classificados por não apresentarem variação significativa na massa específica do fluido. Seu estudo, também conhecido como hidrodinâmica, em geral se concentra nos meios líquidos, sendo que para gases consideram-se incompressíveis aqueles cujo número de Mach é inferior a 0,3 (POTTER; WIGGERT, 2009).

Nas últimas décadas, os avanços na ciência dos materiais tem proporcionado a execução de estruturas cada vez mais esbeltas e flexíveis, capazes de desenvolver grandes deslocamentos sem atingir a ruptura. Tais estruturas, no entanto, podem apresentar efeitos que muitas vezes não são previstos ou considerados de forma inadequada na etapa de projeto quando expostos a um meio fluido. Como exemplo, pode-se citar o amplamente conhecido colapso da ponte de Tacoma Narrows em novembro de 1940, ilustrada na Fig.1.1 ${ }^{1}$, que ruiu devido a um fenômeno conhecido como flutter.

Com o amplo desenvolvimento da simulação numérica, impulsionada principalmente pela expansão da informática pós II Guerra Mundial, tanto a mecânica dos fluidos computacional como a mecânica das estruturas computacional vêm ganhando destaque. Juntamente com o aumento do desempenho das máquinas, a simulação computacional em problemas mecânicos vem se mostrando cada vez mais vantajosa, não somente devido ao aspecto financeiro, mas também pelo grau de confiabilidade dos resultados obtidos.

1 Disponível em: http://www.magnusmundi.com/tacoma-narrows-a-ponte-que-desabou/. Acesso em jan. 2016. 


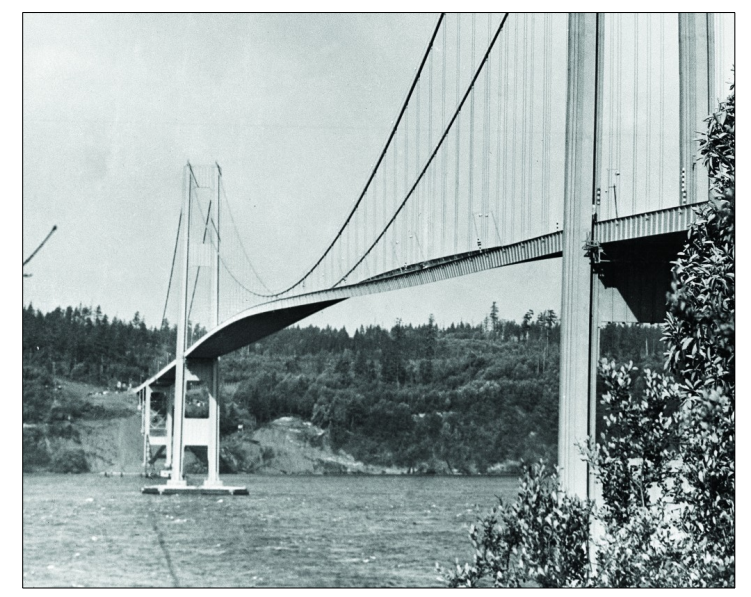

(a)

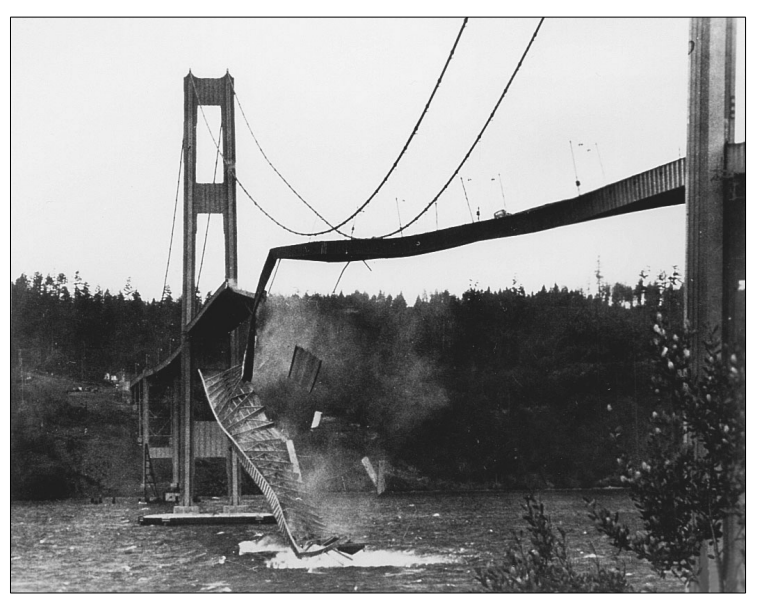

(b)

Figura 1.1: Ponte de Tacoma Narrows (a) instantes antes do colapso e (b) após a ruína.

Fonte: Magnus Mundi.

Neste trabalho, tem-se como propósito a investigação do problema de acoplamento particionado fraco entre fluidos incompressíveis e estruturas de casca que possam desenvolver grandes deslocamentos, dando continuidade ao trabalho de Sanches (2011). Para tanto é desenvolvido um código computacional com formulação baseada no método de elementos finitos para análise de escoamentos incompressíveis em descrição Lagrangeana-Euleriana arbitrária (ALE), o qual é acoplado a um programa já existente de análise dinâmica não-linear geométrica de cascas (CODA; PACCOLA, 2009; SANCHES; CODA, 2013).

Para melhor compreensão do leitor acerca dos temas abordados neste trabalho, o texto encontra-se organizado da seguinte maneira:

- Capítulo 1 - Introdução: Neste capítulo é realizada uma apresentação, bem como uma breve revisão bibliográfica sobre os temas do trabalho. Tendo como base o Estado da Arte, são delimitados os objetivos bem como a justificativa deste trabalho;

- Capítulo 2 - Mecânica dos fluidos incompressíveis: Inicialmente são apresentadas as equações governantes da dinâmica dos fluidos em descrição Euleriana. Em seguida, o caso incompressível isotérmico é particularizado e as equações governantes são finalmente estendidas para a abordagem Lagrangeana-Euleriana Arbitrária;

- Capítulo 3 - Mecânica dos sólidos: Os fundamentos da mecânica dos sólidos são apresentados, tais como a cinemática de um sólido deformável, bem como a relação que define seu equilíbrio dinâmico;

- Capítulo 4 - Análise numérica dos fluidos incompressíveis: As equações governantes dos escoamentos incompressíveis isotérmicos são resolvidas empregando-se o MEF e a metodologia é investigada através da simulação de exemplos numéricos; 
- Capítulo 5 - Análise numérica da mecânica dos sólidos: O MEF posicional é aplicado ao elemento de casca e testes numéricos são realizados para verificar sua eficiência;

- Capítulo 6 - Acoplamento fluido-casca: O modelo de acoplamento particionado fraco é aplicado para realizar a interação fluido-casca, que tem seus resultados verificados por meio da comparação com exemplos da literatura;

- Capítulo 7 - Conclusão: São feitas as considerações finais sobre o desenvolvimento do trabalho, bem como as possibilidades de avanço da pesquisa.

\subsection{Conceituação do problema de interação fluido-estrutura}

Os problemas de interação fluido-estrutura no campo da aeroelasticidade foram descritos por Collar (1946) considerando-se três tipos de forças: a primeira é resultante do escoamento, sendo estudada pela mecânica dos fluidos (forças aerodinâmicas); a segunda se refere à mecânica dos sólidos (forças elásticas); e, finalmente, a última força está relacionada à dinâmica das estruturas (forças inerciais). Para compreender melhor a forma como estes três tipos de força são consideradas na modelagem do problema físico, Collar (1946) propôs o diagrama apresentado da Fig. 1.2.

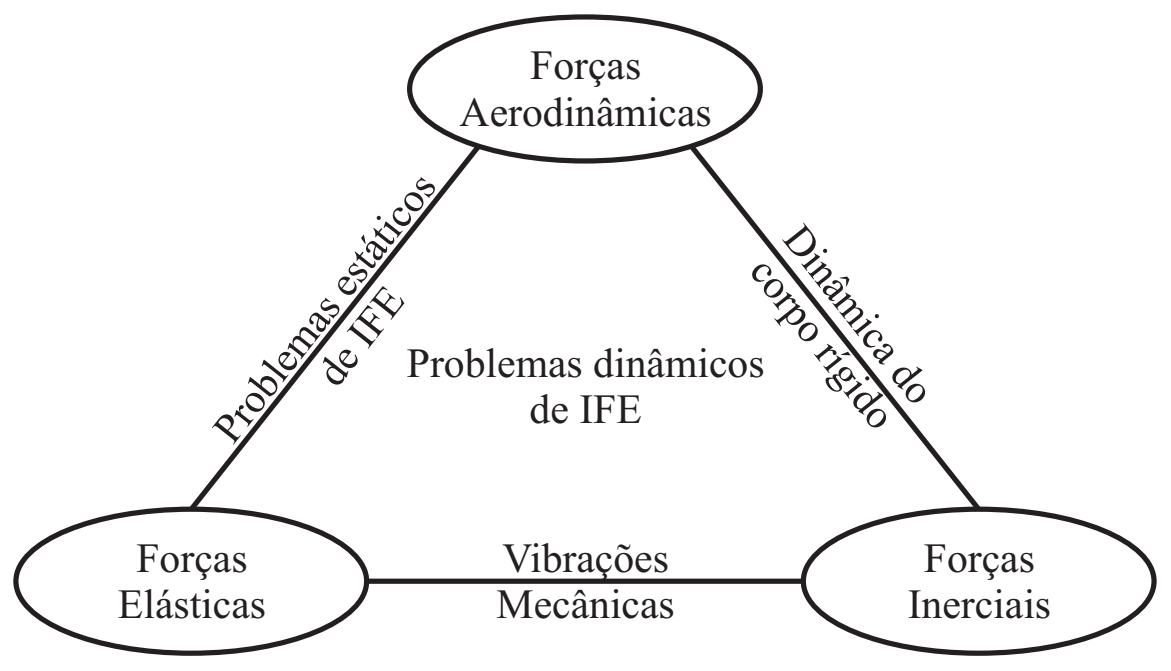

Figura 1.2: Diagrama de Collar

Através do Diagrama de Collar, observa-se que problemas envolvendo a ação de forças aerodinâmicas e elásticas configuram caso estático de interação fluido-estrutura, que se mostra interessante quando a movimentação do sólido é bastante lenta ou amortecida.

O estudo da associação de forças elásticas e inerciais, no entanto, se mostra importante a partir do momento em que a interação com o meio fluido não é significativa, tal como nos problemas de vibrações livres ou forçadas de estruturas robustas. A combinação de forças 
aerodinâmicas e inerciais, por sua vez, é relevante no estudo de rajadas e de carregamentos aerodinâmicos.

Por fim, e mais importante para este trabalho, a combinação das três forças consiste num problema dinâmico de interação fluido-estrutura. Em tais casos os deslocamentos da estrutura, provocados ou não pelas forças do fluido, interferem no escoamento, acarretando num fenômeno acoplado de grande complexidade.

Ainda sobre a classificação dos problemas de IFE, tanto os fenômenos estáticos quanto os dinâmicos podem ser divididos em dois grupos: problemas de resposta ou de estabilidade. No primeiro grupo, encontram-se aqueles efeitos que ocorrem para qualquer velocidade de escoamento, ao passo que os problemas de estabilidade se manifestam a partir de determinada velocidade, chamada de crítica (SANCHES, 2011). Para melhor esclarecimento, alguns exemplos de problemas aeroelásticos são listados na Tabela 1.1, seguidos de uma breve abordagem sobre como se manifestam.

Tabela 1.1: Classificação dos problemas aeroelásticos

\begin{tabular}{cll}
\hline Problema & Estático & Dinâmico \\
\hline \multirow{2}{*}{ Estabilidade } & Divergência & Flutter \\
& & Stall Flutter \\
\hline \multirow{2}{*}{ Resposta } & Distribuição de Carregamento & Resposta a rajadas \\
& Efetividade de controle & Resposta a comandos \\
& Reversão de controle & Buffeting \\
\hline \multicolumn{2}{c}{ Fonte: Cunha (2004). }
\end{tabular}

\subsubsection{Problemas estáticos de resposta}

Estes eventos ocorrem quando a estrutura sofre deformações estáticas tais que impeçam o seu funcionamento correto. Um dos exemplos desse efeito é a reversão de controle em que, ao ser inclinado, o ailerão da aeronave age no sentido de aumentar a sua sustentação. Quando esta inclinação não é suficiente, ao invés de ganhar sustentação, a estrutura pode perdê-la, dependendo de sua rigidez (HIRSCH, 1994).

Outro efeito que se enquadra nessa classificação é a Distribuição de Carregamento, em que a deformação excessiva da estrutura pode levar a situações de carregamento não previstas em projeto, ocasionando por vezes seu colapso.

\subsubsection{Problemas dinâmicos de resposta}

Como exemplo de problema dinâmico de resposta, o caso mais conhecido é o buffeting. Esse fenômeno é caracterizado pelo regime de vibração forçada ocasionada pelo desprendimento de esteiras de vórtices à montante da estrutura, como ilustrado na Fig. 1.3. 


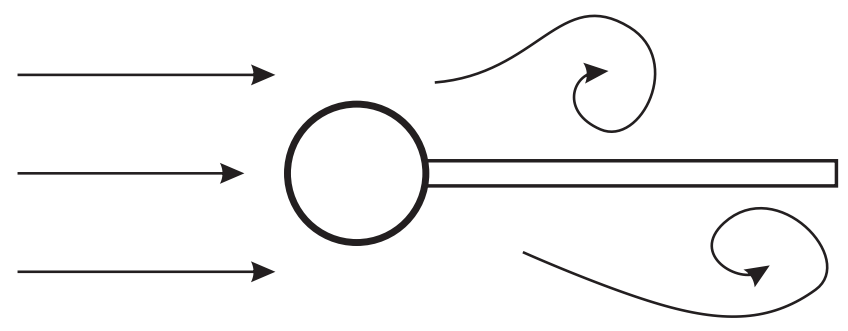

Figura 1.3: Buffeting ou martelamento

Este fenômeno é de difícil modelagem, pois o desprendimento de vórtices pode ocorrer de forma estocástica. No caso de vórtices com desprendimento periódico, a frequência com que atua pode coincidir com uma das frequências naturais da estrutura, levando-a à ressonância. Contudo, mesmo em casos onde a ressonância não é atingida, os problemas relativos à fadiga do material mostram-se de grande importância (HIRSCH, 1994).

\subsubsection{Problemas estáticos de estabilidade}

Nesta categoria enquadra-se o fenômeno de divergência. Para exemplificá-lo, apresentase na Fig. 1.4 um esquema estrutural simplificado comumente empregado no estudo de asas de aviões. Neste caso, quando se tem uma velocidade $U$ baixa e uma rigidez à torção $K_{\theta}$ alta, a deflexão desenvolvida pela mola é muito pequena. Entretanto, para uma dada velocidade crítica $\mathrm{U}_{D}$ (velocidade de divergência), ocorre perda de estabilidade, sendo que qualquer perturbação causaria a saída da configuração atual de equilíbrio (DOWELL, 2015).

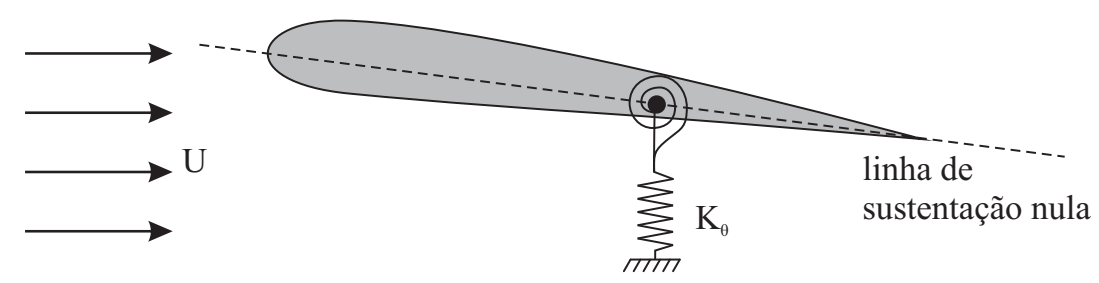

Figura 1.4: Esquema estrutural simplificado de uma asa de avião

Fonte: Adaptado de Hodges e Pierce (2011).

\subsubsection{Problemas dinâmicos de estabilidade}

O exemplo mais comum deste tipo de fenômeno é o flutter, já destacado anteriormente como o efeito que levou ao colapso da ponte de Tacoma Narrows. O flutter ocorre devido ao acoplamento do movimento dinâmico da estrutura com o escoamento do fluido fazendo com que, a partir de certo momento, a amplitude do movimento causado por uma pequena perturbação aumente indefinidamente. De modo geral, em estruturas como pontes e asas, esse problema 
é caracterizado pela ação conjunta dos modos de flexão e torção, com a resposta dinâmica dominada por vibrações torsionais. Diferentemente do efeito de buffeting, o flutter se manifesta a partir de uma velocidade crítica de escoamento e depende pouco do amortecimento e muito da geometria escolhida para a estrutura (HIRSCH, 1994).

\subsection{Estado da arte}

Para que o tema proposto nesta pesquisa seja melhor compreendido, é importante que se tenha uma base teórica consolidada. Para tanto, deve-se ter em mente que a modelagem da IFE necessariamente compreende a união de três problemas: a dinâmica dos fluidos computacional, a dinâmica das estruturas computacional e o problema de acoplamento. Desse modo, é realizada a seguir uma breve revisão bibliográfica sobre os avanços científicos ocorridos nas últimas décadas em cada uma dessas áreas.

\subsubsection{Dinâmica dos Fluidos Computacional}

A diferença entre o comportamento mecânico dos sólidos e dos fluidos situa-se, essencialmente, no fato de que os últimos não são capazes de resistir a tensões desviadoras quando estão em repouso. Do ponto de vista da aproximação numérica, portanto, fica evidente que tanto a mecânica dos sólidos quanto a mecânica dos fluidos possuem particularidades que necessitam de atenção (ZIENKIEWICZ; TAYLOR; NITHIARASU, 2005).

Muitos trabalhos dedicaram esforços significativos para desenvolver métodos estáveis para a dinâmica dos fluidos computacional, principalmente no que diz respeito aos métodos numéricos das Diferenças Finitas e dos Volumes Finitos. Mais recentemente, a partir da década de 1970 o MEF passou a ser mais estudado no contexto da mecânica dos fluidos por pesquisadores renomados, resultando em um grande número de trabalhos de alto nível e ganhando espaço por diversos aspectos, tal como a utilização de malhas não-estruturadas completamente arbitrárias que podem facilitar na representação de domínios irregulares, além da simplicidade na imposição de condições de contorno em fronteiras de alta ordem (ZIENKIEWICZ; TAYLOR; NITHIARASU, 2005; REDDY; GARTLING, 2010; CHUNG, 2002; ANDERSON, 1995).

Diferentemente da mecânica dos sólidos, o MEF sofreu certa resistência para começar a ser utilizado na mecânica dos fluidos, sendo aplicado pela primeira vez à análise de escoamentos viscosos incompressíveis em meados da década de 1970. Em problemas estruturais, é comum que se trabalhe com elementos de treliça, viga, chapas, cascas etc., de modo que em poucos casos necessita-se da resolução de problemas em meios contínuos e mesmo quando necessário, seu tratamento é facilmente realizado por meio do MEF. No caso dos fluidos no entanto, exige-se uma análise bidimensional ou tridimensional para que se tenha uma boa representação do fenômeno em estudo. Além disso, algumas características das equações governantes da mecânica dos fluidos fizeram com que a aceitação do MEF nesse contexto não fosse imediata (ZIENKIEWICZ; 
TAYLOR; NITHIARASU, 2005).

Nos problemas de elasticidade, tem-se um funcional de energia com princípio de mínimo onde, dependendo do método variacional utilizado (ex.: resíduos ponderados, Princípio dos Trabalhos Virtuais ou Método de Ritz para minimização do funcional de energia), se obtém uma matriz de rigidez simétrica de boa aproximação. Ao contrário, muitos problemas na dinâmica dos fluidos podem apresentar convecção dominante, com matrizes assimétricas e o aparecimento de oscilações espúrias nos resultados quando da aplicação do esquema clássico de Galerkin sobre uma descrição Euleriana (BROOKS; HUGHES, 1982; ZIENKIEWICZ; TAYLOR; NITHIARASU, 2005; TEIXEIRA; AWRUCH, 2005; CHUNG, 2002; STRANG; FIX, 2008).

Esse problema é reduzido com o refinamento da malha. Entretanto, um método eficiente deve ser capaz de tratar o problema de forma estável mesmo em uma malha pouco refinada. Nesse sentido, foram desenvolvidas algumas alterações no processo de Galerkin, que em suma consistem na adição de uma difusividade artificial ao problema, capaz de suprimir as variações espúrias. Uma forma tradicional (métodos “upwind") consiste em utilizar funções de peso de ordem diferente das funções de interpolação (processo de Petrov-Galerkin).

Brooks e Hughes (1982) introduziram um processo desenvolvido totalmente a partir de um princípio variacional chamado Streamline Upwind Petrov-Galerkin - SUPG, que consiste no emprego do processo de Petrov-Galerkin, escolhendo funções ponderadoras que adicionam difusividade na direção das linhas de corrente. Tezduyar e Senga (2006), Akin (2004), Catabriga e Coutinho (2002) também apresentam estudos relevantes nesse tema, consolidando o SUPG como uma técnica reconhecidamente eficiente em substituição ao processo de Galerkin.

$\mathrm{Na}$ simulação de escoamentos incompressíveis por meio do MEF existes diversas formas de tratamento das equações governantes, seja pela substituição das variáveis primitivas (formulações vorticidade-linha de corrente), ou ainda pela sua manutenção (formulação mista). $\mathrm{Na}$ abordagem denominada "mista", o método de Galerkin é aplicado diretamente às equações governantes mantendo-se suas variáveis primitivas: velocidade e pressão. Esta abordagem requer a utilização de funções de interpolação diferentes para a aproximação das variáveis do problema (velocidade e pressão) para evitar instabilidades, o que implica na condição de LadyzhenskayaBabuška-Brezzi, ou LBB (ZIENKIEWICZ; TAYLOR; NITHIARASU, 2005; CHUNG, 2002; STRANG; FIX, 2008). Isso deve ser levado em consideração no momento da escolha do elemento finito e do algoritmo de integração temporal a ser empregado.

Outro aspecto importante na análise numérica de dinâmica dos fluidos consiste nos efeitos devido à turbulência. Desde que a discretização espacial contemple todas as escalas que se deseja captar, as equações de Navier-Stokes são capazes de simular com precisão escoamentos turbulentos. No entanto, o que se busca atualmente são métodos eficientes e capazes de representar tais fenômenos com um menor custo computacional. Neste sentido, algumas técnicas consistem na proposição de modelos de turbulência algébricos, que podem ser baseados na hipótese de Reynolds (Reynolds Averaged Navier-Stokes-RANS), além das 
simulações de grandes vórtices (Large Eddy Simulation-LES) (LAUNDER; SPALDING, 1972; WILCOX, 1993; BAZILEVS et al., 2007; NITHIARASU; LIU, 2006).

É importante ressaltar que nos últimos anos diversos métodos numéricos com base em elementos finitos vêm sendo desenvolvidos para simulações em dinâmica dos fluidos. Como exemplo, tem-se os métodos variacionais multiescala, de partículas (Particle finite element methods - PFEM) e os métodos de Galerkin descontínuo (discontinuous Galerkin methods).

Os métodos variacionais multiescala, introduzidos por Hughes (1995), Hughes et al. (1998), Hughes, Mazzei e Jansen (2000) e Hughes, Oberai e Mazzei (2001) propõem a decomposição do problema físico em grandes e pequenas escalas, resolvendo-as separadamente. Como os escoamentos turbulentos são caraterizados por um intervalo de escalas muito amplo, estes métodos têm se mostrado bastante promissores nesta área, aliando-se também à conceitos da simulação de grandes vórtices (Large-Eddy Simulation) (JOHN; KAYA, 2005; BAZILEVS; KOROBENKO; YAN, 2015).

Em contraste com a tradicional abordagem Euleriana no tratamento de problemas de dinâmica dos fluidos computacional, os métodos de partículas (PFEM), primeiramente proposto por Gingold e Monaghan (1997) e desenvolvidos a partir dos métodos sem malha (meshless methods), vêm sendo desenvolvidos em descrição Lagrangeana. Essa forma de tratamento das equações governantes tem se mostrado favorável em análises de problemas cujo domínio sofre alterações constantemente, tais como escoamentos com superfície livre, incluindo problemas de IFE com condições complexas de contato (IDELSOHN; OÑATE; PIN, 2004; IDELSOHN et al., 2008; DÁVALOS et al., 2015).

O método de Galerkin descontínuo, por sua vez, possui vantagem de não gerar matrizes globais, o que além de reduzir o uso de memória necessária para a resolução do problema, possibilita melhor aproveitamento de plataformas de programação paralelas. Entretanto, como este método baseia-se na relaxação da continuidade entre os elementos, há um acréscimo no número de graus de liberdade gerados para a resolução de um problema com o mesmo número de elementos em relação a um método contínuo, por exemplo. Reporta-se também sua ineficiência em termos de tempo de processamento para a resolução de problemas estacionários envolvendo transferência de calor ou difusão em relação ao métodos contínuos (LI, 2006; ZIENKIEWICZ et al., 2003).

\subsubsection{Dinâmica das Estruturas Computacional}

A utilização do método dos elementos finitos na solução de problemas remonta da década de 1960. Sua aplicação à engenharia de estruturas parece ter sido uma escolha natural, uma vez que os engenheiros da época já costumavam realizar a análises subdividindo-se a estrutura em elementos e determinando seu comportamento localmente. O advento dos métodos numéricos, no entanto, permitiu que se pudesse compreender melhor tanto os efeitos globais quanto em regiões de interesse (ZIENKIEWICZ; TAYLOR, 2000).

Impulsionados por uma base algébrica bem consolidada por trabalhos como os de 
Malvern (1969), Ogden (1984), Belytschko, Schwer e Klein (1977), Argyris et al. (1978), Argyris et al. (1979) e Crisfield (1991), por exemplo, o MEF veio a se tornar o método numérico mais empregado para a simulação computacional de sólidos.

Com o amadurecimento do MEF, diversas abordagens foram desenvolvidas. Uma delas é a corrotacional proposta inicialmente por Truesdell (1955) e que segundo Belo (2009) tem como princípio descrever a cinemática do sólido decompondo os movimentos do corpo em rígido e de deformação. Como exemplos de progresso da abordagem corrotacional, podem-se citar os trabalhos de Hughes e Liu (1981a), Simo e Fox (1989), Ibrahimbegovic e Taylor (2002), Argyris (1982) Battini e Pacoste (2006) e Bathe (1996).

Como nesta abordagem as rotações em torno de cada eixo são consideradas como parâmetros nodais, apesar de eficiente para pequenos deslocamentos, em muitos casos não se pode aplicar a comutatividade de rotações em grandes deslocamentos. Dessa forma, nas formulações corrotacionais aplicam-se, por exemplo, as fórmulas de Euler-Rodrigues linearizadas para giro finito (ver Coda e Paccola (2010) para maiores detalhes).

Neste trabalho, optou-se pela utilização de elementos finitos de cascas, uma vez a maioria dos problemas estruturais de interesse podem ser simulados com a utilização destes elementos a um custo computacional menor do que com elementos sólidos. Com relação ao MEF na análise não linear geométrica de elementos de casca, os trabalhos de Hughes e Carnoy (1983), Hughes e Liu (1981a), Hughes e Liu (1981b) e Brendel e Ramm (1980) também merecem ser destacados.

Mais recentemente, Bonet et al. (2000) e Coda (2003) introduziram uma alternativa Lagrangeana e que contempla intrinsecamente os efeitos da não linearidade geométrica em sólidos e estruturas. Ao contrário dos métodos tradicionais, a formulação dita posicional utiliza as posições e inclinações finais de vetores inicialmente normais como parâmetros nodais ao invés de deslocamentos e rotações, o que o torna bastante robusto, mas de simples implementação, como atestam os trabalhos de Coda e Paccola (2007), Coda (2009a), Coda (2009b).

Por se tratar de uma abordagem Lagrangeana total e devido à forma como é realizado o mapeamento para a configuração deformada, análises dinâmicas com base no MEF posicional apresentam matriz de massa constante, o que permite a utilização do integrador temporal de Newmark, garantindo conservação da quantidade de movimento e apresentando conservação de energia suficiente para garantir estabilidade nos problemas usuais como atestam os trabalhos de Coda e Paccola (2009) e Sanches e Coda (2013).

A análise de cascas através da formuação posicional foi apresentada por Coda e Paccola (2007) e estendida para o caso dinâmico por Coda e Paccola (2009). Neste trabalho, também pretende-se utilizar a formulação posicional, que mais recentemente vem sendo empregada em problemas de IFE, como nos trabalhos de Sanches (2011), Sanches e Coda (2013) e Sanches e Coda (2014), comprovando sua eficiência para esta aplicação. 


\subsubsection{Problema de Acoplamento}

Os problemas de dinâmica das estruturas caracterizam-se por desenvolverem deformações finitas. Assim, uma descrição Lagrangeana, ou material, se mostra bastante adequada, tendo como incógnitas principais os deslocamentos ou posições. Por outro lado, os fluidos (Newtonianos) não apresentam resistência às tensões tangenciais, podendo se deformar indefinidamente. Desse modo, uma formulação Euleriana, ou espacial, com velocidades como variáveis principais torna-se mais adequada para a maior parte dos problemas. Tais particularidades implicam na necessidade de se acoplar duas descrições matemáticas distintas quando do estudo de problemas de interação fluido-estrutura.

Uma estratégia bastante difundida é a resolução do fluido empregando-se a descrição Lagrangeana-Euleriana arbitrária (ALE), onde o domínio físico (computacional) pode se mover arbitrariamente e independentemente do movimento da partícula do fluido (DONEA; GIULIANI; HALLEUX, 1982).

As análises de interação fluido-estrutura que se desenvolvem com base na descrição ALE são também conhecidas como métodos de malhas conforme, assim denominadas por realizar o acoplamento através de condições de contorno entre os domínios e necessitar que a malha se ajuste às variações geométricas da interface. Nestes casos, além de resolver os problemas físicos do fluido e do sólido, deve-se levar em consideração a movimentação da malha, o que se torna uma desvantagem da técnica (HOU; WANG; LAYTON, 2012).

Um aspecto que deve ser levado em conta na modelagem da dinâmica dos fluidos em domínios móveis é a lei de conservação geométrica (LCG), proposta inicialmente por Thomas e Lombard (1979) e também abordada nos trabalhos de Lesoinne e Farhat (1996), Farhat, Lesoinne e Maman (1995) e Koobus e Farhat (1999). Essa lei estabelece que as equações governantes discretas num volume de controle devem ser conservativas no tempo, de modo que sua integração temporal deve fornecer a solução exata para um escoamento uniforme. Contudo, Boffi e Gastaldi (2004) e Formaggia e Nobile (2004) demonstram que o atendimento à LCG não é condição necessária nem suficiente para a estabilidade, exceto em casos como o método de Euler. Para alguns casos, Morton, Melville e Visbal (1998) demonstram também que os mesmos resultados são obtidos respeitando-se ou não a LCG.

Quanto ao acoplamento, os problemas multi-físicos tais como o de interação fluidoestrutura podem ser modelados por duas diferentes abordagens: monolítica ou particionada. Na abordagem monolítica, utilizada em trabalhos como os de Blom (1998), Hron e Madlik (2007) e Hübner, Walhorn e Dinkler (2004), ambos os domínios são integrados numa única etapa. Nos métodos de acoplamento particionado, tais como os trabalhos de Felippa, Park e Farhat (2001), Teixeira e Awruch (2005), Sanches e Coda (2010a), Sanches e Coda (2010b), Sanches (2011) e Piperno (1997), as equações governantes são integradas no tempo separadamente e o acoplamento entre os domínios é realizado por meio da transferência de forças e velocidades.

A principal vantagem dos métodos particionados em relação aos monolíticos encontra-se na modularidade. Isso significa que há a possibilidade de avanço paralelo dos algoritmos de 
resolução do fluido e da estrutura, uma vez que um é completamente independente do outro. Além disso, ao resolver um único sistema de equações, caso da abordagem monolítica, o custo computacional é aumentado quando comparado a dois subsistemas (FELIPPA; PARK; FARHAT, 2001; HEIL; HAZEL; BOYLE, 2008).

Como desvantagem do esquema particionado, destaca-se a defasagem que pode ocorrer entre as integrações temporais do fluido e da estrutura quando da atualização explícita das condições na interface fluido-estrutura, o que não ocorre nos esquemas monolíticos, além de instabilidades numéricas como o efeito de massa adicionada (added-mass effect) (FELIPPA; PARK; FARHAT, 2001; BRUMMELEN, 2009).

Nos métodos particionados, o acoplamento entre os domínios é realizado pela transferência de condições de contorno entre os domínios. No esquema clássico de Dirichlet-Neumann, são aplicadas condições de Dirichlet no fluido (velocidades, advindas da movimentação da estrutura) e de Neumann no sólido (forças, advindas da variação nos campos de pressão e das tensões viscosas). Podem ser ainda classificados em dois grupos: forte e fraco. O último caracterizase pela escolha de um passo de tempo suficientemente pequeno para que o acoplamento notoriamente explícito entre os domínios seja numericamente estável. Já nos métodos de acoplamento particionado forte, para um mesmo passo de tempo são realizadas iterações de correção, tornando o procedimento implícito (ROUX; GARAUD, 2009).

Como mencionado anteriormente, os métodos de acoplamento particionado estão sujeitos à instabilidade denominada efeito de massa adicionada. Tallec e Mouro (2001) demonstram que, para um escoamento governado essencialmente pelo campo de pressão, o fluido age na estrutura como uma massa adicional, alterando sua inércia. Essa massa adicional geralmente não se mostra significativa em escoamentos compressíveis de alta velocidade. No entanto, em escoamentos incompressíveis, principalmente se as densidades do sólido e do fluido são próximas ou quando a estrutura é muito esbelta, são observadas instabilidades quando da utilização de métodos de acoplamento particionado fraco (CAUSIN; GERBEAU; NOBILE, 2005; BRUMMELEN, 2009).

Uma técnica para contornar o efeito de massa adicionada desenvolvida recentemente consiste em alterar o esquema de acoplamento do tipo Dirichlet-Neumann para condições de contorno de Robin, isto é, combinações lineares das condições de Dirichlet e Neumann (CAUSIN; GERBEAU; NOBILE, 2005; NOBILE; VERGARA, 2008; BURMAN; FERNÁNDEZ, 2014). Essa técnica tem se mostrado bastante promissora, como é demonstrado por Badia, Nobile e Vergara (2008) que obtiveram resultados estáveis utilizando um esquema Robin-Neumann, isto é, alterando apenas as condições de contorno impostas ao fluido do tipo Dirichlet para Robin.

\subsection{Objetivos}

Este trabalho em como objetivo principal o desenvolvimento de ferramentas computacionais de modo a contribuir para a formação de uma plataforma precisa e eficiente de solução numérica de problemas de interação fluido-estrutura a baixas velocidades de escoamento com 
base no método dos elementos finitos. Dentro deste escopo, alguns objetivos específicos podem ser listados como etapas a serem vencidas ao longo do desenvolvimento do trabalho:

i. Elaboração de revisão bibliográfica visando estudo aprofundado da dinâmica não linear geométrica das estruturas, com enfoque em formulações com elementos de alta ordem livres da aproximação de grandes rotações;

ii. Estudo aprofundado da dinâmica dos fluidos incompressível com ênfase em novas técnicas de estabilização e desenvolvimento de algoritmo baseado em elementos finitos que seja eficiente e permita acoplamento com sólidos flexíveis;

iii. Desenvolvimento de programa para análise tridimensional de escoamentos incompressíveis em descrição ALE;

$i v$. Implementação de modelo de acoplamento particionado (fraco) entre o programa desenvolvido para análise de escoamentos incompressíveis com o programa de dinâmica não linear de cascas já existente, desenvolvido por Coda e Paccola (2007) e Coda e Paccola (2008);

v. Estudo da eficiência do modelo implementado objetivando-se tanto a validação da metodologia quanto a identificação de problemas de natureza numérica que possam afetar o desempenho e a precisão;

\subsection{Metodologia}

Para se obter um programa estável em relação à mecânica dos fluidos e que permita contornos móveis, são utilizados elementos finitos tetraédricos de aproximação quadrática para velocidade e linear para pressão, com as equações em uma descrição Lagrangeana-Euleriana Arbitrária (ALE). Além disso, a técnica SUPG (Streamline-Upwind/Petrov-Galerkin) é utilizada para a estabilização da formulação no que diz respeito às variações espúrias que podem ocorrer quando do emprego do método de Galerkin sobre os termos convectivos.

Quanto à dinâmica não linear geométrica de cascas, uma abordagem Lagrangeana total pelo método dos elementos finitos posicional é empregada. É adotada a cinemática de Reissner para a descrição do elemento finito em uma técnica que substitui deslocamentos e rotações nodais por posições e componentes de um vetor generalizado, com integração temporal realizada por meio do método de Newmark- $\beta$.

O acoplamento fluido-casca implementado é do tipo particionado e ALE-Lagrangeano de forma a garantir a modularidade do programa. Para a movimentação da malha do fluido, desenvolve-se uma metodologia com base em uma malha grossa auxiliar de ordem cúbica com deslocamentos distribuídos pela equação de Laplace.

No que se refere à implementação computacional, tanto o programa de dinâmica dos fluidos quanto o de cascas dinâmicas foram implementados em linguagem de programação Fortran 77 em ambiente LINUX. Em relação à análise não linear dinâmica de cascas, o programa 
utilizado foi desenvolvido de forma serial por Coda e Paccola (2008) e paralelizado por Kawabata et al. (2009) em protocolo MPI (Message Passing Interface). O programa para análise dinâmica de fluidos incompressíveis é implementado de forma sequencial utilizando-se a biblioteca HSL (Harwell Subroutine Library), por meio do pacote HSL_MP42 para a solução do sistema linear que também utiliza processamento paralelo com padrão de comunicação MPI.

Para a análise dos resultados, empregam-se os programas para visualização científica Kitware ParaView e GNUplot.

\subsection{Justificativa}

A evolução das tecnologias computacionais nas últimas décadas vem dando destaque à simulação numérica de problemas de engenharia. Com o advento de computadores cada vez mais potentes, tem sido possível simular problemas cada vez mais complexos com tempo de processamento e uso de recursos cada vez menor.

Contudo, os problemas de interação fluido-estrutura se mostram ainda mais desafiadores, pois envolvem complexidades que vão além da resolução dos problemas de fluido e de sólido separadamente. Além do mais, a grande maioria destes problemas não têm solução analítica satisfatória. Os que possuem, demandam de simplificações exageradas gerando, muitas vezes, resultados pouco confiáveis ou de baixa aplicabilidade, além de serem inviáveis quando se possui um domínio de alta complexidade. Por outro lado, as análises experimentais são muito dispendiosas, requerem espaço, equipamentos de ponta e possuem aplicação limitada ao tipo de problema e geometria simulados.

Desse modo, este trabalho busca introduzir o estudo da interação fluido-estrutura incompressível na recentemente criada linha de pesquisa em IFE do Departamento de Engenharia de Estruturas (SET), dando sequência aos trabalhos de Sanches (2011), Sanches e Coda (2010a), Sanches e Coda (2010b), Sanches e Coda (2013) e Sanches e Coda (2014). 
CAPÍtULO

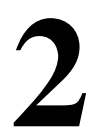

10

\section{MECÂNICA DOS FLUIDOS \\ INCOMPRESSÍVEIS}

O comportamento dinâmico de um fluido é função de suas componentes de velocidade e tensão, além da temperatura e massa específica ao longo do tempo. Neste capítulo, é feita uma breve introdução à descrição matemática da dinâmica dos fluidos incompressíveis, primeiramente em descrição Euleriana, admitindo-se as hipóteses de meio contínuo e comportamento newtoniano. Em sequência, as equações governantes também são descritas na forma LagrangeanaEuleriana Arbitrária, permitindo a análise de escoamentos contemplando a movimentação do domínio de referência de forma arbitrária.

\subsection{Conservação da massa}

A lei de conservação da massa implica essencialmente na indestrutibilidade da matéria, sendo admissível na grande maioria dos problemas de engenharia. Essa lei enuncia que a massa de um sistema deve permanecer constante ao logo do tempo. Portanto, o balanço do fluxo de massa entrando e saindo de um volume de controle $(V C)$ qualquer, deve ser igual à variação da massa dentro do $V C$ ao longo do tempo:

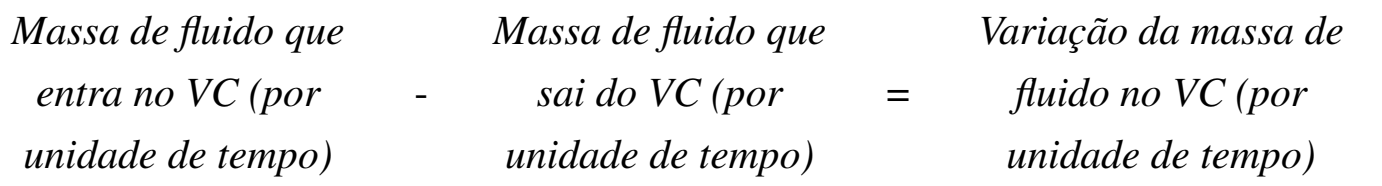

ou ainda, em termos da vazão mássica $\dot{m}$ :

$$
\dot{m}_{\text {entrada }}-\dot{m}_{\text {saída }}=\dot{m}=\frac{\partial m}{\partial t},
$$

em que $m$ é a massa de fluido contida no volume de controle e $t$ é o tempo.

Tomando um volume de controle infinitesimal, como na Fig. 2.1, é possível determinar a 


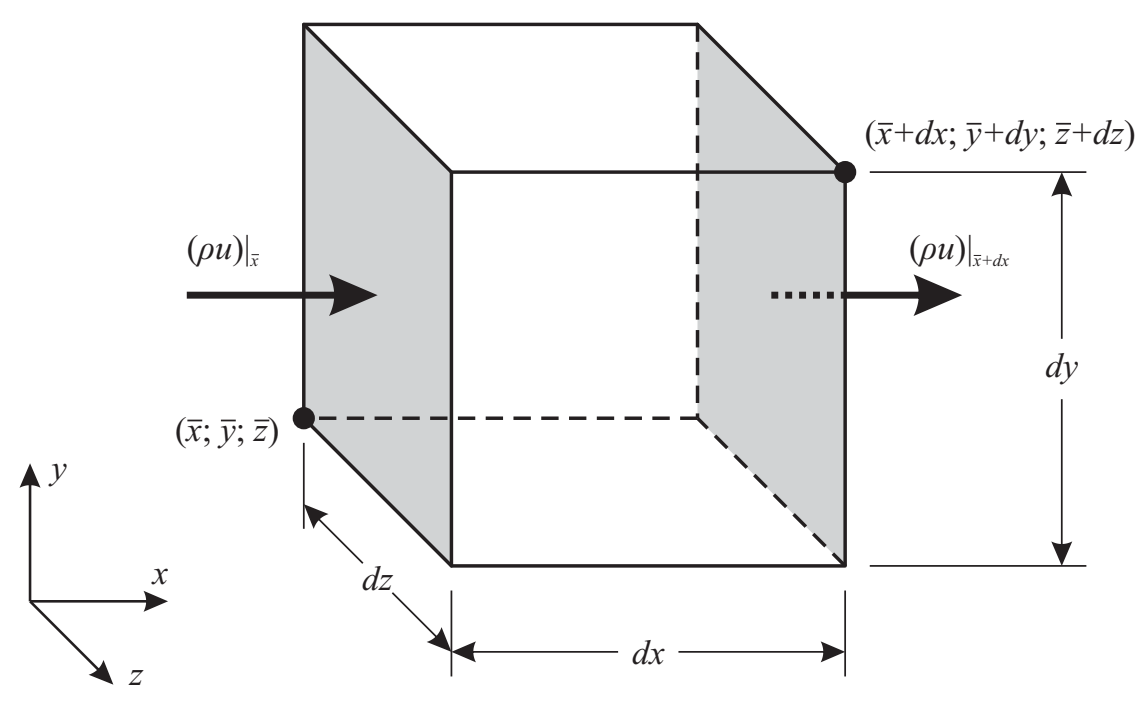

Figura 2.1: Volume de controle infinitesimal e balanço do fluxo de massa

variação da massa ao longo do tempo em função da massa específica do fluido $(\rho)$ :

$$
\frac{\partial m}{\partial t}=\frac{\partial \rho}{\partial t} d x d y d z
$$

em que $d x, d y$ e $d z$ são as dimensões do volume de controle infinitesimal.

O campo de velocidades de um fluido pode ser representado vetorialmente como $\mathbf{u}=(u) \widehat{i}+(v) \hat{j}+(w) \widehat{k}$, com $\widehat{i}, \widehat{j}$ e $\widehat{k}$ indicando os versores normais e $u, v$ e $w$ as respectivas componentes de velocidade nas direções cartesianas. Tomando apenas a direção horizontal $x$ na Fig. 2.1, pode-se determinar a vazão mássica nas faces de entrada e saída de fluxo como:

$$
\dot{m}_{\text {entrada }}^{x}=\left.(\rho u)\right|_{\bar{x}} d y d z, \quad \dot{m}_{\text {saída }}^{x}=\left.(\rho u)\right|_{\bar{x}+d x} d y d z
$$

ou ainda:

$$
\dot{m}_{\text {saída }}^{x}=\left(\left.(\rho u)\right|_{\bar{x}}+\left.\frac{\partial}{\partial x}(\rho u)\right|_{\bar{x}} d x\right) d y d z .
$$

Pela arbitrariedade de $\bar{x}$, considerando as direções $y$ e $z$ e substituindo na Eq. 2.2, obtémse:

$$
\begin{array}{r}
(\rho u) d y d z-\left(\rho u+\frac{\partial}{\partial x}(\rho u) d x\right) d y d z+(\rho v) d x d z-\left(\rho v+\frac{\partial}{\partial y}(\rho v) d y\right) d x d z \\
+(\rho w) d x d y-\left(\rho w+\frac{\partial}{\partial z}(\rho w) d z\right) d x d y=\frac{\partial \rho}{\partial t} d x d y d z
\end{array}
$$

Somando os termos comuns e dividindo a equação pelo volume $d V=d x d y d z$, chega-se a equação que descreve a conservação da massa, também conhecida como equação da continuidade:

$$
\frac{\partial \rho}{\partial t}+\frac{\partial}{\partial x}(\rho u)+\frac{\partial}{\partial y}(\rho v)+\frac{\partial}{\partial z}(\rho w)=0
$$


ou ainda, em notação indicial:

$$
\frac{\partial \rho}{\partial t}+\frac{\partial\left(\rho u_{i}\right)}{\partial x_{i}}=0, \operatorname{com} i=1,2,3
$$

\subsection{Conservação da quantidade de movimento}

A quantidade de movimento de um corpo é definida como o produto entre sua massa $m$ e velocidade u. A Segunda Lei de Newton, por sua vez, diz que um corpo tende a variar sua quantidade de movimento quando submetido à ação de forças externas, isto é:

$$
\mathbf{F}=\left.\frac{D \mathbf{P}}{D t}\right|_{\text {sistema }},
$$

em que $\mathbf{F}$ é a força resultante, $D() / D t$ é a derivada material, ou substancial e $\mathbf{P}$ é o momentum linear do sistema, definido por

$$
\mathbf{P}=\int_{\text {sistema }} \mathbf{u} d m
$$

Ao substituir a Eq. 2.10 em 2.9, obtém-se a relação geral que define a conservação da quantidade de movimento de um corpo qualquer:

$$
\sum \mathbf{F}_{s}+\sum \mathbf{F}_{g}=\frac{D(m \cdot \mathbf{u})}{D t} .
$$

em que a força resultante $\mathbf{F}$ é substituída pelos somatórios de forças de campo, ou de domínio $\mathbf{F}_{g}$ e das forças de superfície $\mathbf{F}_{s}$.

Em um volume de controle infinitesimal, como o ilustrado na Fig. 2.2, tem-se a variação da quantidade de movimento para a componente de velocidade $u$, por exemplo, dada por:

$$
\left.\frac{D(m u)}{D t}\right|_{\bar{x}}=\left(\left.\frac{\partial(\rho u)}{\partial t}\right|_{\bar{x}}+\left.\frac{\partial(u \rho u)}{\partial x}\right|_{\bar{x}}+\left.\frac{\partial(v \rho u)}{\partial y}\right|_{\bar{x}}+\left.\frac{\partial(w \rho u)}{\partial z}\right|_{\bar{x}}\right) d x d y d z .
$$

Para simplificar a representação, denomina-se de $g_{x}$ a resultante das forças de domínio na direção $x$. No caso das forças de superfície, são compreendidas as tensões de origem viscosa, além da tensão hidrostática, ou pressão, atuando sobre o fluido. Realizando dessa forma o somatório das forças na direção $x$, por exemplo, tem-se:

$$
\begin{aligned}
\left.d F_{x}\right|_{\bar{x}}=\left[\left.\rho g_{x}\right|_{\bar{x}}-\left.\left(\sigma_{x x}\right)\right|_{\bar{x}}+\left.\left(\sigma_{x x}\right)\right|_{\bar{x}+d x}\right. & -\left.\left(\tau_{x y}\right)\right|_{\bar{y}}+\left.\left(\tau_{x y}\right)\right|_{\bar{y}+d y} \\
& \left.-\left.\left(\tau_{x z}\right)\right|_{\bar{z}}+\left.\left(\tau_{x z}\right)\right|_{\bar{z}+d z}\right] d x d y d z,
\end{aligned}
$$

ou ainda,

$$
\begin{aligned}
\left.d F_{x}\right|_{\bar{x}} & =\left[\left.\rho g_{x}\right|_{\bar{x}}-\left.\left(\sigma_{x x}\right)\right|_{\bar{x}}+\left.\left(\sigma_{x x}\right)\right|_{\bar{x}}+\left.\frac{\sigma_{x x}}{\partial x}\right|_{\bar{x}}\right. \\
& \left.-\left.\left(\tau_{x y}\right)\right|_{\bar{y}}+\left.\left(\tau_{x y}\right)\right|_{\bar{y}}+\left.\frac{\tau_{x y}}{\partial y}\right|_{\bar{y}}-\left.\left(\tau_{x z}\right)\right|_{\bar{z}}+\left.\left(\tau_{x z}\right)\right|_{\bar{z}}+\left.\frac{\tau_{x z}}{\partial z}\right|_{\bar{z}}\right] d x d y d z,
\end{aligned}
$$

Tomando a definição do tensor de tensões de Cauchy $\boldsymbol{\sigma}$, tem-se: 


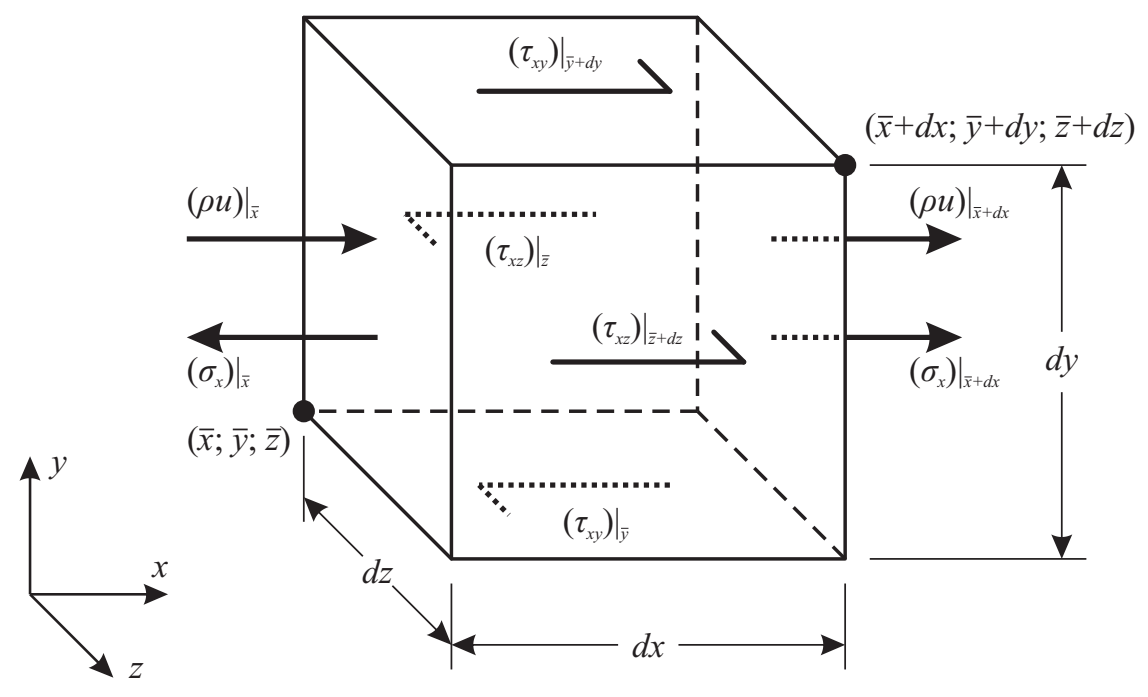

Figura 2.2: Volume de controle infinitesimal e balanço do fluxo da quantidade de movimento

$$
\boldsymbol{\sigma}=\left[\begin{array}{ccc}
\sigma_{x x} & \tau_{x y} & \tau_{x z} \\
\tau_{y x} & \sigma_{y y} & \tau_{y z} \\
\tau_{z x} & \tau_{z y} & \sigma_{z z}
\end{array}\right]=\left[\begin{array}{ccc}
\tau_{x x} & \tau_{x y} & \tau_{x z} \\
\tau_{y x} & \tau_{y y} & \tau_{y z} \\
\tau_{z x} & \tau_{z y} & \tau_{z z}
\end{array}\right]-\left[\begin{array}{ccc}
p & 0 & 0 \\
0 & p & 0 \\
0 & 0 & p
\end{array}\right],
$$

substituindo $\sigma_{x x}$ em 2.14 e simplificando os termos comuns, tem-se:

$$
d F_{x}=\left(\left.\rho g_{x}\right|_{\bar{x}}-\left.\frac{\partial p}{\partial x}\right|_{\bar{x}}+\left.\frac{\partial \tau_{x x}}{\partial x}\right|_{\bar{x}}+\left.\frac{\partial \tau_{x y}}{\partial y}\right|_{\bar{y}}+\left.\frac{\partial \tau_{x z}}{\partial z}\right|_{\bar{z}}\right) d x d y d z
$$

em que $p$ é a pressão e $\tau_{i j}$ são as tensões desviadoras.

Por fim, substituindo-se as Eq. 2.12 e 2.16 em 2.11 e considerando a arbitrariedade de $\bar{x}, \bar{y}$ e $\bar{z}$, tem-se a relação que define a conservação da quantidade de movimento de uma partícula de fluido para a direção $x$ :

$$
\frac{\partial \rho u}{\partial t}+\frac{\partial(u \rho u)}{\partial x}+\frac{\partial(v \rho u)}{\partial y}+\frac{\partial(w \rho u)}{\partial z}=\rho g_{x}-\frac{\partial p}{\partial x}+\frac{\partial \tau_{x x}}{\partial x}+\frac{\partial \tau_{x y}}{\partial y}+\frac{\partial \tau_{x z}}{\partial z} .
$$

Repetindo o procedimento de forma análoga para as direções $y$ e $z$, obtém-se por fim:

$$
\begin{gathered}
\frac{\partial \rho v}{\partial t}+\frac{\partial(u \rho v)}{\partial x}+\frac{\partial(v \rho v)}{\partial y}+\frac{\partial(w \rho v)}{\partial z}=\rho g_{y}-\frac{\partial p}{\partial y}+\frac{\partial \tau_{y x}}{\partial x}+\frac{\partial \tau_{y y}}{\partial y}+\frac{\partial \tau_{y z}}{\partial z} \\
\frac{\partial \rho w}{\partial t}+\frac{\partial(u \rho w)}{\partial x}+\frac{\partial(v \rho w)}{\partial y}+\frac{\partial(w \rho w)}{\partial z}=\rho g_{z}-\frac{\partial p}{\partial z}+\frac{\partial \tau_{z x}}{\partial x}+\frac{\partial \tau_{z y}}{\partial y}+\frac{\partial \tau_{z z}}{\partial z}
\end{gathered}
$$

ou em notação indicial:

$$
\frac{\partial \rho u_{i}}{\partial t}+\frac{\partial\left(u_{j} \rho u_{i}\right)}{\partial x_{j}}=\rho g_{i}-\frac{\partial p}{\partial x_{i}}+\frac{\partial \tau_{i j}}{\partial x_{j}}, \operatorname{com} i, j=1,2,3 .
$$




\subsection{Conservação da energia}

A lei de conservação de energia, ou $1^{\text {a }}$ lei da termodinâmica, enuncia que a energia de um sistema fechado deve permanecer constante ao longo do tempo. Dentre as diversas formas de energia conhecidas, neste trabalho limita-se a realizar este balanço considerando apenas as energias potencial, cinética e interna, resultando na seguinte relação:

$$
\frac{\partial(\rho E)}{\partial t}=-\frac{\partial\left(\rho E u_{j}\right)}{\partial x_{j}}+\frac{\partial}{\partial x_{i}}\left(k \frac{\partial T}{\partial x_{i}}\right)-\frac{\partial\left(p u_{j}\right)}{\partial x_{j}}+\frac{\partial\left(\tau_{i j} u_{j}\right)}{\partial x_{j}}+\rho g_{i} u_{i}, \operatorname{com} i, j=1,2,3,
$$

em que $E$ é a energia específica, $k$ é a constante de condutividade térmica e $T$ é a temperatura absoluta do fluido.

\subsection{Definição do problema incompressível isotérmico}

Ao analisar as equações governantes, pode-se perceber que para o caso tridimensional, se possuem 5 equações: conservação da massa, conservação da quantidade de movimento para cada uma das três direções cartesianas e a conservação da energia. Contudo, o problema possui 15 incógnitas, sendo elas pressão, massa específica, temperatura, 3 componentes de velocidade e 9 componentes de tensão desviadora. Isso faz com que seja impossível resolver o problema da forma como foi proposta até aqui. Assim, a estratégia que se adota é limitar o campo de aplicações e, consequentemente, a simplificação da análise.

Nesse sentido, considerou-se primeiramente apenas o estudo de escoamentos incompressíveis isotérmicos. Um escoamento isotérmico é aquele em que a variação da temperatura do fluido ao longo do tempo é desprezível. A propriedade incompressível, por sua vez, indica que a massa específica do fluido não deve variar significativamente ao longo do tempo ou ainda, para gases, que o número de Mach seja da ordem de 0,3 ou inferior:

$$
M=\frac{u_{\infty}}{c},
$$

onde $u_{\infty}$ é a velocidade de referência do escoamento e $c$ a velocidade de propagação do som no fluido.

Essa primeira hipótese faz com que as equações da conservação da quantidade de movimento e da energia se tornem desacopladas, sendo desnecessária a resolução da última. Com a consideração de massa específica constante, a Eq. 2.7 passa a assumir a seguinte forma:

$$
\frac{\partial u}{\partial x}+\frac{\partial v}{\partial y}+\frac{\partial w}{\partial z}=\frac{\partial u_{i}}{\partial x_{i}}=\nabla \cdot \mathbf{u}=0
$$

sendo que $\nabla \cdot \mathbf{u}$ representa a operação divergência sobre o vetor de velocidades $\mathbf{u}$.

Por outro lado, este estudo contempla apenas fluidos Newtonianos, isto é, aqueles cujas tensões viscosas são proporcionais à taxa de deformação por cisalhamento, ou angular, e a viscosidade dinâmica do fluido $(\mu)$, de forma que a componente $\sigma_{i j}$ do tensor de Cauchy é dada por: 


$$
\sigma_{i j}=-p \delta_{i j}+\lambda \frac{\partial u_{k}}{\partial x_{k}} \delta_{i j}+\mu\left(\frac{\partial u_{i}}{\partial x_{j}}+\frac{\partial u_{j}}{\partial x_{i}}\right)
$$

onde $\delta_{i j}$ é o delta de Kronecker e $\lambda$ é um parâmetro constitutivo que segundo a hipótese de Stokes vale $-2 / 3 \mu$. Ao observar a Eq. 2.23, no entanto, nota-se que fluidos incompressíveis possuem divergência nula. Isso acaba por anular a parcela onde $\lambda$ está inserido, fazendo com que seu valor não tenha importância nesse caso.

Desse modo, a consideração das três hipóteses anteriores faz com que o problema de escoamento incompressível isotérmico em descrição Euleriana tenha, para o caso tridimensional, 4 incógnitas (3 componentes de velocidade e pressão) e 4 equações: a conservação da massa (Eq. 2.23) e a conservação da quantidade de movimento, também conhecidas como Equações de Navier-Stokes, reescritas como:

$$
\begin{gathered}
\rho\left(\frac{\partial u}{\partial t}+u \frac{\partial u}{\partial x}+v \frac{\partial u}{\partial y}+w \frac{\partial u}{\partial z}\right)=\rho g_{x}-\frac{\partial p}{\partial x}+\mu\left(\frac{\partial^{2} u}{\partial x^{2}}+\frac{\partial^{2} u}{\partial y^{2}}+\frac{\partial^{2} u}{\partial z^{2}}\right) \\
\rho\left(\frac{\partial v}{\partial t}+u \frac{\partial v}{\partial x}+v \frac{\partial v}{\partial y}+w \frac{\partial v}{\partial z}\right)=\rho g_{y}-\frac{\partial p}{\partial y}+\mu\left(\frac{\partial^{2} v}{\partial x^{2}}+\frac{\partial^{2} v}{\partial y^{2}}+\frac{\partial^{2} v}{\partial z^{2}}\right) \\
\rho\left(\frac{\partial w}{\partial t}+u \frac{\partial w}{\partial x}+v \frac{\partial w}{\partial y}+w \frac{\partial w}{\partial z}\right)=\rho g_{z}-\frac{\partial p}{\partial z}+\mu\left(\frac{\partial^{2} w}{\partial x^{2}}+\frac{\partial^{2} w}{\partial y^{2}}+\frac{\partial^{2} w}{\partial z^{2}}\right)
\end{gathered}
$$

ou ainda, em notação indicial:

$$
\rho\left(\frac{\partial u_{i}}{\partial t}+u_{j} \frac{\partial u_{i}}{\partial x_{j}}\right)-\rho g_{i}+\frac{\partial p}{\partial x_{i}}-\mu \frac{\partial}{\partial x_{j}}\left(\frac{\partial u_{i}}{\partial x_{j}}+\frac{\partial u_{j}}{\partial x_{i}}\right)=0, \text { com } i, j=1,2,3 \text {. }
$$

\subsection{Equações governantes na descrição Lagrangeana-Euleriana Arbitrária (ALE)}

Um dos desafios enfrentados ao trabalhar com problemas de interação fluido-estrutura se dá pela necessidade de acoplamento de duas descrições matemáticas distintas. Como já mencionado, o sólido, por possuir deformações finitas geralmente é descrito em uma formulação Lagrangeana, ou seja, o movimento de cada partícula é acompanhado ao longo do tempo. Entretanto, os fluidos podem se deformar indefinidamente por não possuírem resistência às tensões tangenciais. Assim, a descrição Euleriana se mostra mais adequada, pois uma porção do contínuo é mantida fixa e as velocidades de cada ponto são atualizadas ao longo do tempo (SANCHES, 2011).

Diante disso, uma das maneiras de contornar esse problema é pela utilização da descrição Lagrangeana-Euleriana Arbitrária (ALE), proposta inicialmente por Donea, Giuliani e Halleux (1982). Para tanto, tomam-se três domínios: $R, C\left(t_{0}\right)$ e $C(t)$, respectivamente domínios de referência, contínuo no tempo inicial e final de acordo com a Fig. 2.3.

$\mathrm{O}$ vetor de coordenadas $\zeta_{i}$ de um ponto no domínio de referência está relacionado à posição inicial da partícula e ao tempo através de uma lei de movimentação definida para o 


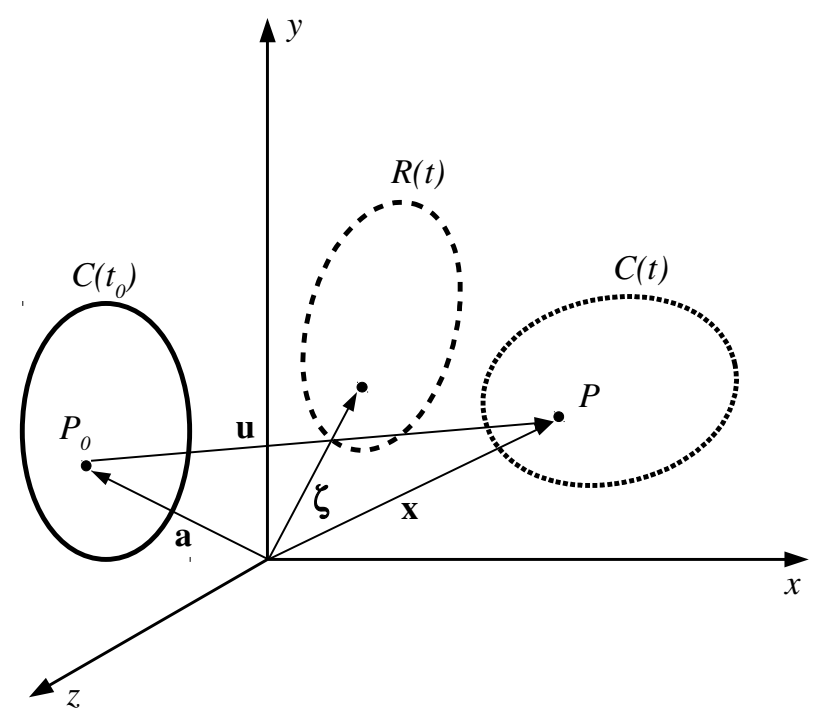

Figura 2.3: Cinemática da descrição ALE.

domínio de referência do tipo:

$$
\zeta_{i}=f_{i}\left(a_{i}, t\right)
$$

Desse modo, a descrição ALE propõe o mapeamento do domínio de referência com relação à sua posição inicial, realizada matematicamente por meio da matriz Jacobiana $\mathbf{J}$ :

$$
J_{i j}=\frac{\partial \zeta_{i}}{\partial a_{j}}
$$

sendo que seu determinante $J$ é chamado de Jacobiano da mudança de configuração e sua variação com o tempo é definida por (ver Donea, Giuliani e Halleux (1982)):

$$
\frac{\partial J}{\partial t}=J \nabla \cdot \boldsymbol{\omega}
$$

em que $\boldsymbol{\omega}$ é o vetor velocidade de movimentação do domínio de referência, tal que $\omega_{i}=\frac{\partial \zeta_{i}}{\partial t}$.

Se considerarmos uma propriedade física qualquer $g\left(\zeta_{i}, t\right)$, expressa na configuração de referência igual a $\tilde{g}$ :

$$
g\left(\zeta_{i}, t\right)=g\left[f_{i}\left(a_{i}, t\right)\right]=\tilde{g}\left(a_{i}, t\right),
$$

pela regra da cadeia, sua derivada com relação ao tempo é definida como:

$$
\left.\frac{\partial \tilde{g}(\mathbf{a}, t)}{\partial t}\right|_{a}=\left.\frac{\partial g(\zeta, t)}{\partial t}\right|_{\zeta}+\frac{\partial g(\zeta, t)}{\partial \zeta_{i}} \omega_{i} .
$$

Utilizando a regra do produto, pode-se definir que:

$$
\nabla \cdot(g \boldsymbol{\omega})=g \nabla \cdot \boldsymbol{\omega}+\boldsymbol{\omega} \cdot \nabla g .
$$


Tendo em vista a Eq. 2.31, pode-se reescrever a regra do produto como:

$$
J \nabla \cdot(g \boldsymbol{\omega})=\frac{\partial J}{\partial t} g+J \boldsymbol{\omega} \cdot \nabla g .
$$

Por fim, manipulando algebricamente a Eq. 2.35 chega-se a:

$$
\frac{\partial}{\partial t}(J \tilde{g})=J\left[\frac{\partial g}{\partial t}+\nabla \cdot(g \boldsymbol{\omega})\right] .
$$

A Eq. 2.36 descreve o transporte de $g$ na descrição ALE e é partindo dessa relação que as equações governantes (Eq. 2.23 e 2.28) podem finalmente ser reescritas (ver Donea, Giuliani e Halleux (1982)), agora na descrição ALE:

$$
\begin{gathered}
\frac{\partial u_{i}}{\partial x_{i}}=0 \\
\rho\left(\frac{\partial u_{i}}{\partial t}+u_{j} \frac{\partial u_{i}}{\partial x_{j}}\right)-\rho g_{i}+\frac{\partial p}{\partial x_{i}}-\mu \frac{\partial}{\partial x_{j}}\left(\frac{\partial u_{i}}{\partial x_{j}}+\frac{\partial u_{j}}{\partial x_{i}}\right)=\rho \omega_{j} \frac{\partial u_{i}}{\partial x_{j}} .
\end{gathered}
$$

Como pode-se observar, quando a velocidade da malha $(\boldsymbol{\omega})$ é igual a zero, recupera-se a descrição Euleriana. Em contrapartida, para $\boldsymbol{\omega}=\mathbf{u}$, tem-se uma descrição Lagrangeana. 
CAPÍtULO
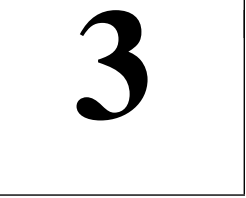

\section{MECÂNICA DOS SÓLIDOS}

Assim como os meios fluidos, os sólidos têm seu movimento governado por três leis básicas: as conservações da massa, da quantidade de movimento e da energia. A partir da hipótese de meio contínuo, este capítulo tem o objetivo de estabelecer uma relação de equilíbrio dinâmico para um sólido em descrição Lagrangeana. Para tanto, o equilíbrio é descrito por meio do funcional de energia mecânica total. Ao fim, aspectos relativos às medidas de deformação e leis constitutivas, pertinentes à consideração dos efeitos de não linearidade geométrica são apresentados.

\subsection{Cinemática de um sólido}

A cinemática é a área da mecânica que estuda o movimento, independente de sua causa. À vista disso, toma-se um ponto arbitrário pertencente a um sólido com carregamentos e vinculações quaisquer num instante de tempo $t_{0}$ no que chamamos de configuração inicial, cuja posição relativa ao sistema de referência é definida pelo vetor $\mathbf{x}$, de acordo com a Fig. 3.1.

Decorrido certo tempo, no instante $t$ o sólido se encontrará na chamada configuração deformada, de modo que o mesmo ponto considerado anteriormente agora encontra-se na posição y. Nota-se que é utilizado um único referencial para mapear a mudança de estado do ponto escolhido, caracterizando uma descrição Lagrangeana total.

Deve haver, portanto, uma função vetorial $\vec{f}$ capaz de representar a trajetória de cada ponto do domínio, que é função de sua posição na configuração inicial e do tempo, tal que:

$$
\mathbf{y}=\vec{f}(\mathbf{x}, t)
$$

Contudo, não é uma tarefa fácil determinar $\vec{f}$ de forma analítica. Desse modo, a utilização de um método numérico eficiente se mostra bastante vantajosa. 


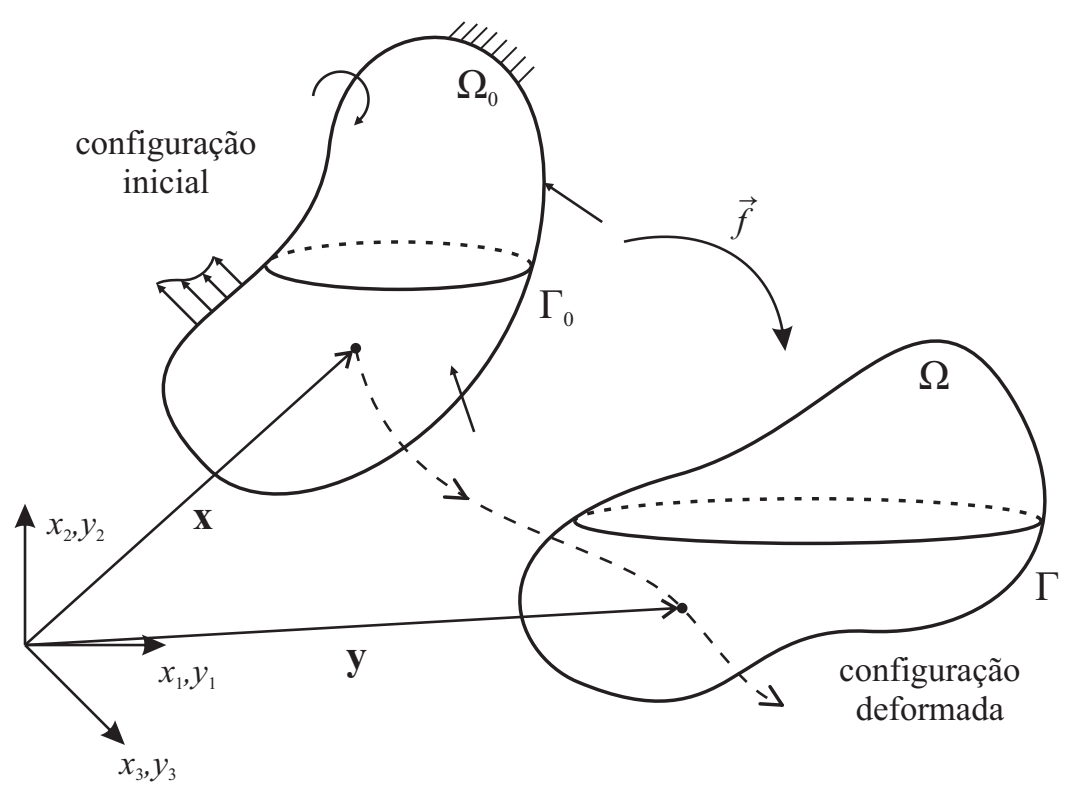

Figura 3.1: Cinemática de um sólido deformável.

\subsection{Equilíbrio de um sólido}

Tendo como base a Eq. 3.1, observa-se que a configuração deformada do corpo deve ser um estado de equilíbrio dinâmico. Assim, é necessário escrever uma relação que permita determinar essa configuração de equilíbrio, agora levando em consideração o estado de carregamentos ao qual o sólido foi previamente submetido.

Para isso, escreve-se o funcional de energia mecânica total de um sólido qualquer (П), omitindo as parcelas geradas por forças dissipativas (amortecimento) ou de origem térmica:

$$
\Pi=\mathbb{P}+\mathbb{K}+U_{e}
$$

A primeira parcela da Eq. 3.2 corresponde ao trabalho realizado pelo carregamento externo e é definida de acordo com:

$$
\mathbb{P}=-\sum \mathbf{F} \cdot \mathbf{y}-\int_{\Omega_{0}}\left(\mathbf{b}_{0} \cdot \mathbf{y}\right) d \Omega_{0}-\int_{\Gamma_{0}}(\mathbf{p} \cdot \mathbf{y}) d \Gamma_{0},
$$

onde $\mathbf{F}$ denota as forças concentradas e $\mathbf{b}_{0}$ e $\mathbf{p}$ representam, respectivamente, as forças de domínio e de superfície. Nessa relação, o sinal negativo ocorre em virtude de as forças externas perderem capacidade de realizar trabalho após exercerem deformação sobre o corpo.

A segunda parcela é referente à energia cinética do sólido e é expressa por:

$$
\mathbb{K}=\int_{\Omega_{0}} \frac{1}{2} \rho_{0}(\dot{\mathbf{y}} \cdot \dot{\mathbf{y}}) d \Omega_{0},
$$

em que $\rho_{0}$ é a massa específica do sólido e o ponto acima da variável indica sua derivada temporal, sendo válida a igualdade $\dot{\mathbf{y}}=\frac{\partial \mathbf{y}}{\partial t}$ para a descrição Lagrangeana.

Por fim, tem-se a fração referente à energia de deformação, resultante da consideração 
do sólido como um meio elástico, definida por:

$$
U_{e}=\int_{\Omega_{0}} u_{e} d \Omega_{0}
$$

sendo que $u_{e}$ é a energia específica de deformação, dependente da relação constitutiva definida para o material.

Assim, o funcional de energia mecânica pode ser reescrito da seguinte forma:

$$
\Pi=-\mathbf{F} \cdot \mathbf{y}-\int_{\Omega_{0}}\left(\mathbf{b}_{0} \cdot \mathbf{y}\right) d \Omega_{0}-\int_{\Gamma_{0}}(\mathbf{p} \cdot \mathbf{y}) d \Gamma_{0}+\int_{\Omega_{0}} \frac{1}{2} \rho_{0}(\dot{\mathbf{y}} \cdot \dot{\mathbf{y}}) d \Omega_{0}+\int_{\Omega_{0}} u_{e} d \Omega_{0} .
$$

Para a determinação da relação de equilíbrio dinâmico, aplica-se o princípio da estacionariedade da energia potencial total, cujo enunciado estabelece que na configuração deformada a primeira variação do funcional de energia deve ser nula para qualquer parâmetro de que П é dependente (LANCZOS, 1986).

Para realizar essa operação, as posições y são escolhidas estrategicamente como parâmetro de variação, pois já estão inserida nas duas primeiras parcelas do funcional e, como será visto adiante no Capítulo 5, o emprego do MEF posicional permite que a energia de deformação também seja determinada em função de y. Procedendo dessa maneira, tem-se:

$$
\begin{aligned}
\delta \Pi & =\int_{\Omega_{0}}\left[\frac{1}{2} \rho_{0} \frac{\partial(\dot{\mathbf{y}} \cdot \dot{\mathbf{y}})}{\partial \mathbf{y}} \cdot \delta \mathbf{y}\right] d \Omega_{0}-\frac{\partial(\mathbf{F} \cdot \mathbf{y})}{\partial \mathbf{y}} \cdot \delta \mathbf{y} \\
& -\int_{\Omega_{0}} \frac{\partial\left(\mathbf{b}_{0} \cdot \mathbf{y}\right)}{\partial \mathbf{y}} \cdot \delta \mathbf{y} d \Omega_{0}-\int_{\Gamma_{0}} \frac{\partial(\mathbf{p} \cdot \mathbf{y})}{\partial \mathbf{y}} \cdot \delta \mathbf{y} d \Gamma_{0}+\int_{\Omega_{0}}\left[\frac{\partial u_{e}}{\partial \mathbf{y}} \cdot \delta \mathbf{y}\right] d \Omega_{0}=0 .
\end{aligned}
$$

Levando em consideração apenas a ação de forças externas conservativas, isto é, independentes da trajetória do corpo, define-se, portanto, a relação de equilíbrio dinâmico de um sólido em forma fraca como:

$$
\begin{aligned}
\delta \Pi & =\int_{\Omega_{0}}\left[\frac{1}{2} \rho_{0} \frac{\partial(\dot{\mathbf{y}} \cdot \dot{\mathbf{y}})}{\partial \mathbf{y}} \cdot \delta \mathbf{y}\right] d \Omega_{0}-\mathbf{F} \cdot \delta \mathbf{y} \\
& -\int_{\Omega_{0}}\left(\mathbf{b}_{0} \cdot \delta \mathbf{y}\right) d \Omega_{0}-\int_{\Gamma_{0}}(\mathbf{p} \cdot \delta \mathbf{y}) d \Gamma_{0}+\int_{\Omega_{0}}\left[\frac{\partial u_{e}}{\partial \mathbf{y}} \cdot \delta \mathbf{y}\right] d \Omega_{0}=0
\end{aligned}
$$

\subsection{Medida de deformação e lei constitutiva}

Para demonstrar o funcionamento de algumas medidas de deformação existentes, Bonet e Wood (2008) propõem a análise do caso unidimensional de uma barra com área constante sendo tracionada por uma força uniforme, como ilustra a Fig. 3.2.

Considerando-se que as seções transversais permanecem planas, perpendiculares ao eixo da barra e indeformáveis em seu plano, uma das formas mais intuitivas de se calcular a deformação da barra, consiste na medida linear de engenharia:

$$
\varepsilon_{E}=\frac{\mathrm{L}-\mathrm{L}_{0}}{\mathrm{~L}_{0}}=\frac{\Delta \mathrm{L}}{\mathrm{L}_{0}} .
$$

Se, no entanto, a variação do comprimento $(\Delta \mathrm{L})$ for dividida pelo comprimento final da 


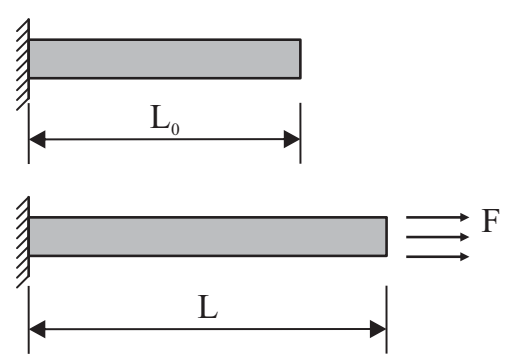

Figura 3.2: Elemento de barra sendo tracionado.

barra na Eq. 3.9, obtém-se a medida de deformação não linear de engenharia:

$$
\varepsilon=\frac{\Delta \mathrm{L}}{\mathrm{L}} .
$$

Por outro lado, a soma dos pequenos incrementos de deformação realizada conforme a barra se deforma, define a medida de deformação natural ou logarítmica:

$$
\varepsilon_{L}=\int_{\mathrm{L}_{0}}^{\mathrm{L}} \frac{1}{s} d s=\ln \left(\frac{\mathrm{L}}{\mathrm{L}_{0}}\right) .
$$

Contudo, embora as medidas de deformação definidas anteriormente possam ser estendidas para análises de sólidos tridimensionais, Bonet e Wood (2008) demonstram que sua generalização além de complexa, é computacionalmente cara. Segundo os mesmos autores, nesses casos, as medidas de deformação de Green $(E)$ e Almansi $\left(\varepsilon_{A}\right)$ se mostram vantajosas, principalmente pela facilidade de extensão ao caso tridimensional:

$$
\begin{gathered}
E=\frac{\mathrm{L}^{2}-\mathrm{L}_{0}^{2}}{2 \mathrm{~L}_{0}^{2}}, \\
\varepsilon_{A}=\frac{\mathrm{L}^{2}-\mathrm{L}_{0}^{2}}{2 \mathrm{~L}^{2}} .
\end{gathered}
$$

É facilmente verificável que quanto menores forem as deformações, isto é, $\mathrm{L} \approx \mathrm{L}_{0}$, mais próximos serão os valores obtidos por ambas as medidas. Entretanto, quando $\mathrm{L} \gg \mathrm{L}_{0}$, cada medida de deformação leva a um resultado completamente diferente.

Do ponto de vista da análise não linear geométrica, algo que se procura em uma medida de deformação é a sua objetividade, ou seja, que os movimentos de corpo rígido não acumulem valores. Este critério também é atingido pela deformação de Green, ou Green-Lagrange, cuja generalização para o caso tridimensional leva à seguinte relação:

$$
\mathbf{E}=\frac{1}{2}(\mathbf{C}-\mathbf{I}),
$$

onde $\mathbf{C}$ representa o alongamento à direita de Cauchy-Green e $\mathbf{I}$ é o tensor identidade de segunda ordem. Aproveitando o conceito de função mudança de configuração introduzido na seção anterior, pode-se demonstrar que $\mathbf{C}$ relaciona-se ao gradiente de $\vec{f}$ (ver Ogden (1984)), tal que:

$$
\mathbf{C}=\mathbf{A}^{T} \mathbf{A}
$$


onde $\mathbf{A}=\operatorname{Grad}(\vec{f})$.

A deformação de Green-Lagrange, por sua vez, é conjugada energética do tensor de tensões de Piola-Kirchhoff (S):

$$
\mathbf{S}=\mathbb{C}: \mathbf{E}
$$

em que $\mathbb{C}$ é o tensor constitutivo de quarta ordem e (:) indica a operação de contração dupla entre os tensores.

Além disso, em análises na mecânica dos sólidos, no que se refere à tensão, deseja-se determinar o estado de tensões real, ou de Cauchy, o qual pode ser determinado a partir das tensões de Piola-Kirchhoff de segunda espécie segundo:

$$
\boldsymbol{\sigma}=\frac{1}{J} \mathbf{A} \mathbf{S} \mathbf{A}^{T}
$$

onde $J$ é o Jacobiano da função mudança de configuração, isto é, $J=\operatorname{det}(\mathbf{A})$.

Quanto ao tipo de material, considera-se o sólido como um material isotrópico hiperelástico do tipo Saint-Venant-Kirchhoff. Segundo esta lei constitutiva, o tensor $\mathbb{C}$ definido anteriormente é dado segundo a expressão:

$$
\mathbb{C}_{i j k l}=\left(\kappa-\frac{2}{3} G\right) \delta_{i j} \delta_{k l}+G\left(\delta_{i k} \delta_{j l}+\delta_{i l} \delta_{j k}\right),
$$

em que $\kappa$ e $G$ são, respectivamente os módulos volumétrico e de cisalhamento (ou de elasticidade transversal), calculados a partir do módulo de elasticidade longitudinal $(\mathbb{E})$ e do coeficiente de Poisson $(v)$ por:

$$
\begin{gathered}
\kappa=\lambda+\frac{2}{3} G \\
G=\frac{\mathbb{E}}{2(1+v)}, \\
\lambda=\frac{v \mathbb{E}}{(1+v)(1-2 v)} .
\end{gathered}
$$

A lei constitutiva de Saint-Venant-Kirchhoff estabelece uma relação linear entre o segundo tensor das tensões de Piola-Kirchhoff e a deformação de Green-Lagrange:

$$
u_{e}^{S V K}=\frac{1}{2} \mathbf{E}: \mathbb{C}: \mathbf{E},
$$

ou ainda, levando-se em consideração a Eq. 3.16:

$$
u_{e}^{S V K}=\frac{1}{2} \mathbf{S}: \mathbf{E} .
$$

Esse modelo se propõe como uma alternativa para a descrição de materiais hiperelásticos, porém deve ser usado apenas em casos de pequenas deformações, pois não impede a degeneração do material quando submetido à grandes deformações de compressão (BAZILEVS; TAKIZAWA; TEZDUYAR, 2013). 
CAPítulo

\section{4}

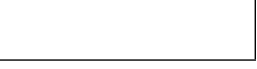

\section{ANÁLISE NUMÉRICA DOS ESCOAMENTOS INCOMPRESSÍVEIS}

Como definido anteriormente, um escoamento incompressível isotérmico é governado pelas leis de conservação da massa e da quantidade de movimento. Tais relações estabelecem um sistema de equações não lineares e diversas estratégias de solução numérica vêm sendo desenvolvidas nas últimas décadas.

Uma técnica bastante empregada consiste na substituição das variáveis primitivas do problema (velocidade e pressão) por expressões contendo vorticidade e linha de corrente. Do ponto de vista dos escoamentos incompressíveis, há um acoplamento forte entre as variáveis primitivas e que pode trazer dificuldades à simulação numérica do problema devido à presença apenas das componentes de velocidade na equação de conservação da massa, tornando a pressão naturalmente implícita. Nesse sentido, a formulação vorticidade-linha de corrente se torna vantajosa por eliminar completamente a pressão da formulação. Entretanto, como desvantagens, tem-se a dificuldade de expansão da formulação para escoamentos tridimensionais, além da maior complexidade na aplicação das condições de contorno em termos da vorticidade (REDDY; GARTLING, 2010).

Outras técnicas, como a estudada neste trabalho, desenvolvem-se a partir das equações governantes em sua versão primitiva, com velocidades e pressões como incógnitas. Mais especificamente, utiliza-se a formulação mista, ou velocidade-pressão, obtida pela aplicação do Método de Galerkin às equações governantes e assim denominado por restringir as variáveis do problema numa única formulação.

Mais adiante, é realizada uma abordagem quanto à estabilidade e convergência do procedimento de solução, bem como aspectos sobre seu avanço temporal. Por fim, a metodologia implementada é submetida a alguns testes numéricos para verificação e validação dos resultados. 


\subsection{Forma fraca e aproximação pelo MEF}

De acordo com os desenvolvimentos do Capítulo 2 procura-se nesta seção inicialmente a forma fraca das Eq. 2.37 e 2.38, isto é, relações integrais a partir das equações diferenciais parciais (EDP's) dadas. Partimos, desse modo, realizando sua integração sobre o domínio de um elemento finito genérico $\left(\Omega^{e}\right)$ segundo o método dos resíduos ponderados:

$$
\begin{gathered}
\int_{\Omega^{e}} \frac{\partial u_{i}}{\partial x_{i}} \mathcal{Q} d \Omega^{e}=0 \\
\int_{\Omega^{e}}\left[\rho\left(\frac{\partial u_{i}}{\partial t}+\left(u_{j}-\omega_{j}\right) \frac{\partial u_{i}}{\partial x_{j}}\right)-\rho g_{i}+\frac{\partial p}{\partial x_{i}}-\mu \frac{\partial}{\partial x_{j}}\left(\frac{\partial u_{i}}{\partial x_{j}}+\frac{\partial u_{j}}{\partial x_{i}}\right)\right] \mathcal{W}_{i} d \Omega^{e}=0,
\end{gathered}
$$

em que $\mathcal{Q}$ e $\mathcal{W}_{i}$ são chamadas funções ponderadoras e são homogêneas nas condições de contorno essenciais, ou de Dirichlet.

Em seguida, procede-se com a integração por partes e aplicação do teorema da divergência à Eq. 4.2, obtendo-se:

$$
\begin{gathered}
\int_{\Omega^{e}} \frac{\partial u_{i}}{\partial x_{i}} \mathcal{Q} d \Omega^{e}=0 \\
\int_{\Omega^{e}}\left\{\rho\left[\mathcal{W}_{i} \frac{\partial u_{i}}{\partial t}+\mathcal{W}_{i}\left(u_{j}-\omega_{j}\right) \frac{\partial u_{i}}{\partial x_{j}}-\mathcal{W}_{i} g_{i}\right]\right. \\
\left.+\frac{\partial \mathcal{W}_{i}}{\partial x_{j}}\left[p \delta_{i j}-\mu\left(\frac{\partial u_{i}}{\partial x_{j}}+\frac{\partial u_{j}}{\partial x_{i}}\right)\right]\right\} d \Omega^{e}-\int_{\Gamma^{e}} \mathcal{W} \mathcal{T}_{i} d \Gamma^{e}=0
\end{gathered}
$$

em que $\mathcal{T}_{i}$ é a componente $i$ da força de superfície no contorno, resultante da integral por partes e definida por:

$$
\mathcal{T}_{i}=\mu\left[2 \frac{\partial u_{i}}{\partial x_{i}} n_{i}+\left(\frac{\partial u_{i}}{\partial x_{j}}+\frac{\partial u_{j}}{\partial x_{i}}\right) n_{j}\right]-p n_{i}
$$

e $n_{i}$ é a componente do vetor normal na direção $i$.

As Eq. 4.3 e 4.4 definem, portanto, a forma fraca do problema incompressível isotérmico. Deste modo, tendo em vista a aproximação clássica de elementos finitos, tem-se a interpolação de cada uma das variáveis do problema (velocidade e pressão) no domínio de um elemento finito expressa por:

$$
\begin{gathered}
u_{i}(x, t)=\sum_{m=1}^{M} \psi_{m}(x) u_{i}^{m}(t)=\Psi^{T} \mathbf{u}_{i} \\
p(x, t)=\sum_{l=1}^{L} \phi_{l}(x) p_{l}(t)=\Phi^{T} \mathbf{p},
\end{gathered}
$$

em que $\mathbf{u}_{i}$ e $\mathbf{p}$ são vetores que contém os valores nodais de velocidade $\left(u_{i}^{m}\right)$ e pressão $\left(p_{l}\right)$ e $\Psi$ e $\Phi$ são chamadas funções de forma ou aproximadoras, por sua vez definidas como:

$$
\Psi^{T}=\left[\begin{array}{llll}
\psi_{1} \mathbf{I} & \psi_{2} \mathbf{I} & \cdots & \psi_{M} \mathbf{I}
\end{array}\right] \text { e } \Phi^{T}=\left[\begin{array}{llll}
\phi_{1} & \phi_{2} & \cdots & \phi_{L}
\end{array}\right],
$$

em que $\phi_{i}$ e $\psi_{i}$ são as funções aproximadoras associadas ao $i$-ésimo nó do elemento finito e I é 
o tensor identidade de dimensão dois ou três para análises bidimensionais ou tridimensionais, respectivamente.

O método de Galerkin (ou Bubnov-Galerkin), por sua vez, propõe o emprego de funções aproximadoras iguais às funções ponderadoras, isto é, $\mathcal{Q}=\Phi$ e $\mathcal{W}_{i}=\Psi$.

Assim, substituem-se as relações definidas nas Eq. 4.6 e 4.7 em 4.3 e 4.4, de forma a obter-se:

$$
\begin{gathered}
-\left[\int_{\Omega^{e}} \Phi \frac{\partial \Psi^{T}}{\partial x_{i}} d \Omega^{e}\right] \mathbf{u}_{i}=0 \\
{\left[\int_{\Omega^{e}} \rho \Psi \Psi^{T} d \Omega^{e}\right] \dot{\mathbf{u}}_{i}+\left[\int_{\Omega^{e}} \rho \Psi \Psi^{T}\left(u_{j}-\omega_{j}\right) \frac{\partial \Psi^{T}}{\partial x_{j}} d \Omega^{e}\right] \mathbf{u}_{i}+\left[\int_{\Omega^{e}} \mu \frac{\partial \Psi}{\partial x_{j}} \frac{\partial \Psi^{T}}{\partial x_{i}} d \Omega^{e}\right] \mathbf{u}_{j}} \\
-\left[\int_{\Omega^{e}} \frac{\partial \Psi^{T}}{\partial x_{i}} \Phi^{T} d \Omega^{e}\right] \mathbf{p}=\left[\int_{\Omega^{e}} \rho \Psi g_{i} d \Omega^{e}\right]+\left[\int_{\Gamma^{e}} \mathcal{T}_{i} \Psi d \Gamma^{e}\right]
\end{gathered}
$$

em que o ponto superposto denota a derivada temporal.

As Eq. 4.9 e 4.10 representam, portanto, o problema incompressível isotérmico no domínio de um elemento finito. Por facilidade de representação, o sistema de equações resultantes pode ser reescrito numa notação compacta como:

$$
\begin{gathered}
-\mathbf{Q}^{\mathbf{T}} \mathbf{u}=\mathbf{0}, \\
\mathbf{M} \dot{\mathbf{u}}+\mathbf{C}(\mathbf{u}-\boldsymbol{\omega}) \mathbf{u}+\mathbf{K u}-\mathbf{Q} \mathbf{p}=\mathbf{F},
\end{gathered}
$$

em que:

$$
\begin{gathered}
\mathbf{M}=\int_{\Omega} \rho \Psi \Psi^{T} d \Omega ; \mathbf{C}(\mathbf{u}-\boldsymbol{\omega})=\int_{\Omega} \rho \Psi \Psi^{T}\left(u_{j}-\omega_{j}\right) \frac{\partial \Psi^{T}}{\partial x_{j}} d \Omega ; \\
\mathbf{K}=\int_{\Omega} \mu \frac{\partial \Psi}{\partial x_{j}} \frac{\partial \Psi^{T}}{\partial x_{i}} d \Omega ; \mathbf{Q}=\int_{\Omega} \frac{\partial \Psi^{T}}{\partial x_{i}} \Phi^{T} d \Omega ; \\
\mathbf{F}=\int_{\Omega} \rho \Psi g_{i} d \Omega+\int_{\Gamma} \mathcal{T}_{i} \Psi d \Gamma .
\end{gathered}
$$

Nas Equações 4.13, 4.14 e 4.15, M e K são chamadas, respectivamente, de matrizes de massa e viscosidade (ou difusão). $\mathbf{Q}$ e $\mathbf{Q}^{\mathbf{T}}$ representam os respectivos operadores gradiente e divergente, $\mathbf{C}(\mathbf{u}-\boldsymbol{\omega})$ compreende os efeitos de convecção (ou advecção) além da movimentação da malha segundo a descrição ALE e $\mathbf{F}$ contém os termos relativos às forças de corpo, ou de domínio (primeira parcela) e de superfície (segunda parcela) aplicadas ao fluido.

Por fim, o sistema de equações resultante é representado matricialmente da seguinte forma:

$$
\left[\begin{array}{cc}
\mathbf{M} & 0 \\
0 & 0
\end{array}\right] \cdot\left\{\begin{array}{l}
\dot{\mathbf{u}} \\
\dot{\mathbf{p}}
\end{array}\right\}+\left[\begin{array}{cc}
\mathbf{K}+\mathbf{C}(\mathbf{u}-\boldsymbol{\omega}) & -\mathbf{Q} \\
-\mathbf{Q}^{\mathbf{T}} & 0
\end{array}\right] \cdot\left\{\begin{array}{l}
\mathbf{u} \\
\mathbf{p}
\end{array}\right\}=\left\{\begin{array}{l}
\mathbf{F} \\
\mathbf{0}
\end{array}\right\} .
$$

É interessante destacar que nessa formulação $\mathbf{p}$ equivale a multiplicadores de Lagrange, com a função de impor a incompressibilidade do fluido (equação da continuidade) (REDDY; GARTLING, 2010). 
No âmbito da aproximação numérica, a formulação mista possui algumas limitações. Por exemplo, na Eq. 4.16 a matriz $\mathbf{Q}^{\mathbf{T}}[\mathbf{K}+\mathbf{C}(\mathbf{u}-\boldsymbol{\omega})] \mathbf{Q}$, também chamada de complemento de Schur, é positiva-definida somente se $\operatorname{ker}(\mathbf{Q})=\{\boldsymbol{0}\}$, ou seja, se o núcleo de $\mathbf{Q}$ for igual ao vetor nulo $\{\boldsymbol{0}\}$. Caso isso aconteça, a matriz da segunda parcela em 4.16 é não-singular e leva a uma solução única e estável para os campos de velocidade e pressão. Caso contrário, pode-se até obter um campo estável para a velocidade, porém o campo de pressão apresenta resposta oscilatória e com variações espúrias (DONEA; HUERTA, 2003).

Para que se tenha um sistema estável, a condição de Ladyzhenskaya-Babuška-Brezzi, ou LBB, deve ser satisfeita. Essa condição matemática relaciona os espaços das funções utilizadas para a aproximação das variáveis do problema (velocidade e pressão) e faz com que a escolha do elemento não seja arbitrária e ainda, que a ordem de aproximação para cada uma das variáveis seja diferente de modo a evitar o aparecimento de modos espúrios de pressão. Desse modo, trabalhos anteriores como o de Zienkiewicz e Taylor (2000) e Donea e Huerta (2003) foram tomados como referência por relacionarem algumas combinações de elementos finitos já testados e que atendem à condição LBB, como ilustra a Fig. 4.1.

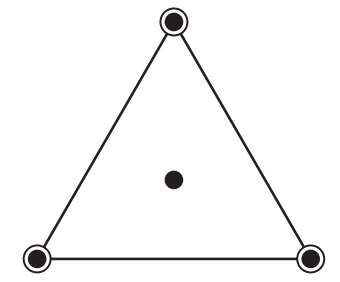

$\mathrm{T} 3 \mathrm{~B} 1 / 3 \mathrm{C}$

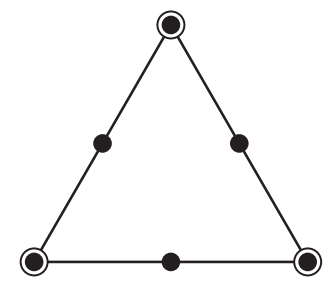

$\mathrm{T} 6 / 3 \mathrm{C}$

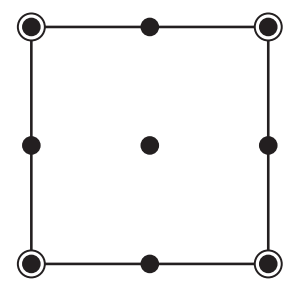

Q9/4C

O Nós que interpolam velocidade e pressão

- Nós que interpolam velocidade

Figura 4.1: Elementos finitos que atendem à condição LBB.

Fonte: Zienkiewicz, Taylor e Nithiarasu (2005).

Neste trabalho, optou-se pela utilização de uma versão tridimensional da combinação T6/3C, que se trata de um elemento tetraédrico isoparamétrico com aproximação quadrática para a velocidade e linear para a pressão, ilustrado na Fig. 4.2.

\subsection{Estabilidade da formulação}

O método clássico de Galerkin, utilizado anteriormente para a obtenção da Eq. 4.16, tem sido empregado na simulação de uma grande variedade de escoamentos incompressíveis, comprovando sua eficiência e robustez. O fato da equação da continuidade e da quantidade de movimento estarem acopladas torna computacionalmente difícil a busca por uma resposta estável, devido principalmente ao tamanho e à forma da matriz a ser invertida. Ao analisar a Eq. 4.16, percebe-se que a matriz $\mathbf{K}+\mathbf{C}(\mathbf{u}-\boldsymbol{\omega})$ é assimétrica, esparsa e positiva semi-definida. 


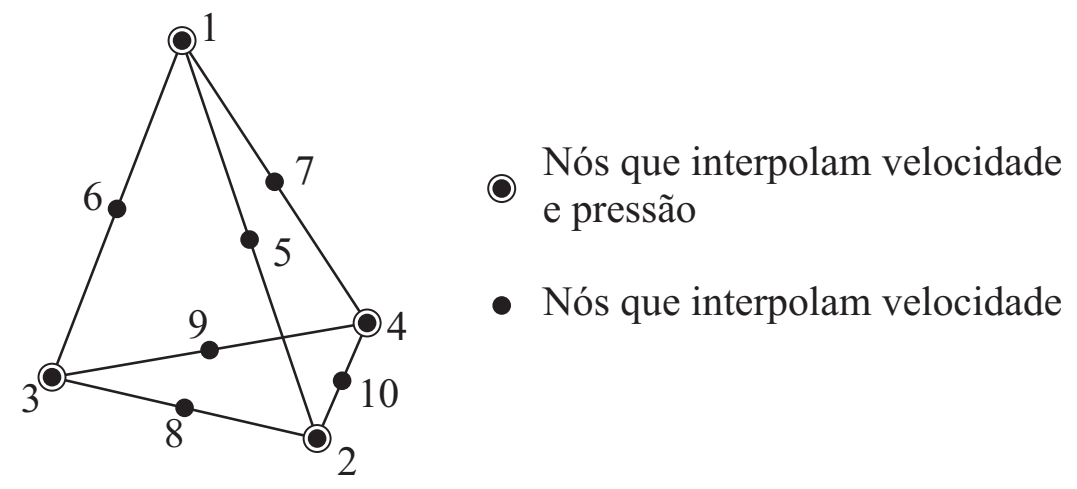

Figura 4.2: Elemento tetraédrico isoparamétrico de aproximação linear para pressão e quadrática para velocidade.

Este tipo de sistemas lineares são característicos de pontos de sela, ou saddle-point problems, problemas que historicamente demandam de grande estudo e esforço para sua resolução. $\mathrm{O}$ uso de algoritmos clássicos para a resolução de sistemas lineares como a eliminação Gaussiana nem sempre retornam respostas estáveis, principalmente devido à presença de zeros nas diagonais que implicam em pivôs nulos (REDDY; GARTLING, 2010; BENZI; GOLUB; LIESEN, 2005).

Desse modo, as pesquisas nessa área vêm concentrando esforços na busca por métodos iterativos e pré-condicionadores adaptados especialmente para esse problema como é o caso por exemplo dos trabalhos de Nigro et al. (1998), Elman, Silvester e Wathen (2002), Melchior et al. (2012), Elman, Silvester e Wathen (2005) .

Neste trabalho, a solução numérica do sistema é obtida a partir do uso da biblioteca HSL (Harwell Subroutine Library). Mais especificamente, utilizou-se o pacote HSL_MP42, que baseia-se na técnica multifrontal, otimizado para problemas envolvendo sistemas de elementos finitos e implementado em uma plataforma paralela com protocolo MPI.

Em um escoamento qualquer, à medida em que sua velocidade diminui, diz-se que ele passa a ser de viscosidade dominante. Isso ocorre porque os termos da matriz $\mathbf{K}$ se tornam numericamente superiores aos da matriz $\mathbf{C}(\mathbf{u})$ e o sistema de equações resultante (Eq. 4.16) se torna cada vez mais linear. Por outro lado, se a velocidade do escoamento aumenta, ele se torna de convecção dominante e, consequentemente, cada vez mais não-linear.

O Método de Galerkin clássico faz com que surjam variações espúrias decorrentes da não-linearidade dos escoamentos de convecção dominante. Para contornar tal problema, vários métodos de estabilização vêm sendo desenvolvidos, sendo que um dos mais antigos e difundidos é o Streamline-Upwind/Petrov-Galerkin (SUPG), proposto inicialmente por Brooks e Hughes (1982). Esse esquema é baseado totalmente na formulação variacional, utilizando o método de Petrov-Galerkin com funções ponderadoras escolhidas de forma a incluir uma viscosidade artificial atuante na direção da velocidade, controlando a magnitude dos termos convectivos nessa direção.

Em termos práticos, essa estabilização consiste no acréscimo de uma parcela à Eq. 4.2: 


$$
\begin{gathered}
\int_{\Omega}\left[\rho\left(\frac{\partial u_{i}}{\partial t}+\left(u_{j}-\omega_{j}\right) \frac{\partial u_{i}}{\partial x_{j}}\right)-\rho g_{i}+\frac{\partial p}{\partial x_{i}}-\mu \frac{\partial}{\partial x_{j}}\left(\frac{\partial u_{i}}{\partial x_{j}}+\frac{\partial u_{j}}{\partial x_{i}}\right)\right] \mathcal{W}_{i} d \Omega \\
+\sum_{e=1}^{n_{e l}} \int_{\Omega} \tau_{S U P G} u_{j} \frac{\partial \mathcal{W}_{i}}{\partial x_{j}}\left[\rho\left(\frac{\partial u_{i}}{\partial t}+u_{j} \frac{\partial u_{i}}{\partial x_{j}}\right)-\rho g_{i}+\frac{\partial p}{\partial x_{i}}-\mu \frac{\partial}{\partial x_{j}}\left(\frac{\partial u_{i}}{\partial x_{j}}+\frac{\partial u_{j}}{\partial x_{i}}\right)\right] d \Omega=0,
\end{gathered}
$$

onde o termo entre colchetes no somatório é chamado de resíduo da Eq. 4.2, $n_{e l}$ é o número de elementos da malha e $\tau_{S U P G}$ é o parâmetro de estabilização da formulação, que é calculado de acordo com os desenvolvimentos de Bazilevs, Takizawa e Tezduyar (2013), através das seguintes relações:

$$
\begin{aligned}
\tau_{S U P G} & =\left(\frac{1}{\tau_{S U G N 1}^{2}}+\frac{1}{\tau_{S U G N 2}^{2}}+\frac{1}{\tau_{S U G N 3}^{2}}\right)^{-1 / 2} \\
\tau_{S U G N 1} & =\left(\sum_{i=1}^{n_{e n}}\left|\mathbf{u} \cdot \nabla \Psi_{i}\right|\right)^{-1}, \\
\tau_{S U G N 2} & =\frac{\Delta t}{2} \\
\tau_{S U G N 3} & =\frac{h_{R G N}^{2}}{4 v}, \\
h_{R G N} & =2\left(\sum_{i=1}^{n_{e n}}\left|\mathbf{r} \cdot \nabla \Psi_{i}\right|\right)^{-1} \\
\mathbf{r} & =\frac{\nabla|\mathbf{u}|}{\|\nabla|\mathbf{u}|\|} .
\end{aligned}
$$

Levando-se em consideração que $\mathcal{W}_{i}=\Psi$, repete-se o procedimento realizado anteriormente, na seção 4.1. Assim, as matrizes locais definidas nas Eq. 4.13, 4.14 e 4.15 podem ser reescritas, desta vez na forma estabilizada de acordo com a técnica SUPG:

$$
\begin{aligned}
\mathbf{M}^{*} & =\int_{\Omega} \rho \Psi \Psi^{T} d \Omega+\sum_{e=1}^{n_{e l}} \int_{\Omega} \tau_{S U P G} \rho \Psi\left(u_{j}\right) \frac{\partial \Psi^{T}}{\partial x_{j}} d \Omega \\
\mathbf{K}^{*} & =\int_{\Omega} \mu \frac{\partial \Psi}{\partial x_{j}} \frac{\partial \Psi^{T}}{\partial x_{i}} d \Omega-\sum_{e=1}^{n_{e l}} \int_{\Omega} \tau_{S U P G} \mu\left(u_{i}\right) \frac{\partial \Psi}{\partial x_{i}} \frac{\partial^{2} \Psi^{T}}{\partial x_{i} \partial x_{j}} d \Omega ; \\
\mathbf{C}^{*}(\mathbf{u}-\boldsymbol{\omega}) & =\int_{\Omega} \rho \Psi \Psi^{T}\left(u_{j}-\omega_{j}\right) \frac{\partial \Psi^{T}}{\partial x_{j}} d \Omega \\
& +\sum_{e=1}^{n_{e l}} \int_{\Omega} \tau_{S U P G} \rho\left(u_{i}-\omega_{i}\right) \frac{\partial \Psi}{\partial x_{i}}\left(u_{j}-\omega_{j}\right) \frac{\partial \Psi^{T}}{\partial x_{j}} d \Omega \\
\mathbf{Q}^{*} & =\int_{\Omega} \frac{\partial \Psi^{T}}{\partial x_{i}} \Phi^{T} d \Omega-\sum_{e=1}^{n_{e l}} \int_{\Omega} \tau_{S U P G}\left(u_{i}\right) \frac{\partial \Psi}{\partial x_{i}}(p) \frac{\partial \Phi^{T}}{\partial x_{j}} d \Omega \\
\mathbf{F}^{*} & =\int_{\Omega} \rho \Psi g_{i} d \Omega+\int_{\Gamma} f_{i} \Psi d \Gamma+\sum_{e=1}^{n_{e l}} \int_{\Omega} \tau_{S U P G} \rho\left(u_{i}\right) \frac{\partial \Psi}{\partial x_{i}} g_{i} d \Omega .
\end{aligned}
$$

Finalmente, pode-se atualizar a Eq. 4.16, agora em uma versão estabilizada: 


$$
\left[\begin{array}{cc}
\mathbf{M}^{*} & 0 \\
0 & 0
\end{array}\right] \cdot\left\{\begin{array}{l}
\dot{\mathbf{u}} \\
\dot{\mathbf{p}}
\end{array}\right\}+\left[\begin{array}{cc}
\mathbf{K}^{*}+\mathbf{C}^{*}(\mathbf{u}-\boldsymbol{\omega}) & -\mathbf{Q}^{*} \\
-\mathbf{Q}^{\mathbf{T}} & 0
\end{array}\right] \cdot\left\{\begin{array}{l}
\mathbf{u} \\
\mathbf{p}
\end{array}\right\}=\left\{\begin{array}{c}
\mathbf{F}^{*} \\
\mathbf{0}
\end{array}\right\} .
$$

ou numa notação simbólica:

$$
\overline{\mathbf{M}} \cdot \dot{\mathbf{U}}+\overline{\mathbf{K}} \cdot \mathbf{U}=\overline{\mathbf{F}}
$$

$\operatorname{com} \dot{\mathbf{U}}=\left\{\dot{\mathbf{u}}_{1}, \dot{\mathbf{u}}_{2}, \dot{\mathbf{u}}_{3}, \dot{\mathbf{p}}\right\}^{T}$ e $\mathbf{U}=\left\{\mathbf{u}_{1}, \mathbf{u}_{2}, \mathbf{u}_{3}, \mathbf{p}\right\}^{T}$.

\subsection{Integração temporal}

Como pode-se avaliar, a Eq. 4.30 representa um problema discreto no espaço e contínuo no tempo. A estratégia de solução numérica de problemas transientes, no entanto, pode ser realizada através do avanço gradual da solução, discretizada no tempo. Ao utilizar tais procedimentos, deve-se levar em consideração aspectos de estabilidade e precisão. Nesse sentido, Reddy e Gartling (2010) apontam que os métodos implícitos, apesar de aparentemente terem maior custo computacional, apresentam vantagens sobre os explícitos ao resolver o problema de escoamento incompressível, basicamente por três razões:

- Implicidade natural da pressão em um fluido incompressível;

- Limitações quanto ao passo de tempo necessário para se obter estabilidade nos esquemas explícitos;

- Ao utilizar modelos explícitos, observam-se problemas de diagonalização e inversão da matriz $\overline{\mathbf{M}}$, acarretando na perda de precisão dos resultados.

Desse modo, dois métodos implícitos foram empregados, um de primeira e outro de segunda ordem, sendo que em ambos é utilizado um esquema de correção do vetor incógnita, com o objetivo de reduzir o número de iterações necessárias para alcançar a convergência a cada passo de tempo.

\subsubsection{Integrador de primeira ordem}

Uma forma eficiente de se resolver o problema de discretização temporal é o método de Euler implícito para o avanço da solução, definido por:

$$
\mathbf{U}^{n+1}(t)=\mathbf{U}^{n}(t)+\Delta t \alpha\left(t^{n+1}, \mathbf{U}^{n+1}\right),
$$

ou ainda,

$$
\mathbf{U}^{n+1}=\mathbf{U}^{n}+\Delta t \dot{\mathbf{U}}^{n+1}
$$

para um problema de valor inicial do tipo 


$$
\alpha(t, \mathbf{U}(t))=\dot{\mathbf{U}}(t), \mathbf{U}\left(t_{0}\right)=\mathbf{U}_{0}
$$

em que $\mathbf{U}^{n}$ e $\mathbf{U}^{n+1}$ representam os respectivos vetores incógnita nos tempos anterior e atual e $\Delta t$ é o passo de tempo, isto é, o intervalo utilizado para a discretização temporal.

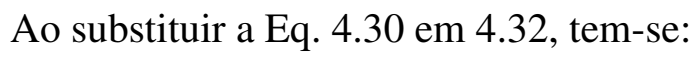

$$
\mathbf{U}^{n+1}=\mathbf{U}^{n}+\Delta t \overline{\mathbf{M}}^{-1}\left[\overline{\mathbf{F}}\left(\mathbf{U}^{n+1}\right)-\overline{\mathbf{K}}\left(\mathbf{U}^{n+1}\right) \mathbf{U}^{n+1}\right]
$$

ou ainda,

$$
\left[\frac{1}{\Delta t} \overline{\mathbf{M}}+\overline{\mathbf{K}}\left(\mathbf{U}^{n+1}\right)\right] \mathbf{U}^{n+1}=\frac{1}{\Delta t} \overline{\mathbf{M}} \mathbf{U}^{n}+\overline{\mathbf{F}}\left(\mathbf{U}^{n+1}\right),
$$

Assim, a Eq. 4.35 define o avanço discreto do problema incompressível de acordo com o método de Euler implícito. Como essa equação é não linear, pode-se recorrer, por exemplo, ao método iterativo de Newton-Raphson para a busca da solução. Tal procedimento consiste em igualar a Eq. 4.35 a um vetor resíduo $\mathbf{R}^{n+1}$. Desde que a solução $\mathbf{U}^{n+1}$ satisfaça a Eq. $4.35, \mathbf{R}^{n+1}$ será igual ao vetor nulo. Como o que se busca é justamente $\mathbf{U}^{n+1}$, inicialmente $\mathbf{R}^{n+1} \neq \mathbf{0}$ :

$$
\left[\frac{1}{\Delta t} \overline{\mathbf{M}}+\overline{\mathbf{K}}\left(\mathbf{U}^{n+1}\right)\right] \mathbf{U}^{n+1}-\frac{1}{\Delta t} \overline{\mathbf{M}} \mathbf{U}^{n}-\overline{\mathbf{F}}\left(\mathbf{U}^{n+1}\right)=\mathbf{R}^{n+1} \neq \mathbf{0} .
$$

Expandindo $\mathbf{R}^{n+1}$ em Série de Taylor e truncando-a nos termos de segunda ordem, tem-se:

$$
\mathbf{R}^{n+1}=\mathbf{R}^{n}+\frac{\partial \mathbf{R}^{n}}{\partial \mathbf{U}^{n}} \Delta \mathbf{U}+\mathcal{O}\left(\Delta \mathbf{U}^{2}\right)
$$

Observando que a condição para que $\mathbf{U}^{n+1}$ satisfaça a Eq. 4.35 é de que $\mathbf{R}^{n+1}=\mathbf{0}$, obtém-se portanto:

$$
\frac{\partial \mathbf{R}^{n}}{\partial \mathbf{U}^{n}} \Delta \mathbf{U}=-\mathbf{R}^{n}
$$

Sendo assim, recorre-se novamente à Eq. 4.36 para a determinação do operador tangente $\frac{\partial \mathbf{R}^{n}}{\partial \mathbf{U}^{n}}$, bem como de $\mathbf{R}^{n}$. Realizando os cálculos adequados, obtém-se por fim:

$$
\left[\frac{1}{\Delta t} \overline{\mathbf{M}}+\overline{\mathbf{K}}\left(\mathbf{U}^{n}\right)+\frac{\partial \overline{\mathbf{K}}\left(\mathbf{U}^{n}\right)}{\partial \mathbf{U}^{n}}\right] \Delta \mathbf{U}=\overline{\mathbf{F}}\left(\mathbf{U}^{n}\right)-\overline{\mathbf{K}}\left(\mathbf{U}^{n}\right) \mathbf{U}^{n}-\frac{1}{\Delta t} \overline{\mathbf{M}}\left[\mathbf{U}^{n}-\mathbf{U}^{n-1}\right] .
$$

Deste modo, para um determinado passo de tempo, a partir da solução anterior $\mathbf{U}^{n}$ calcula-se, através da Eq. 4.39, um incremento $\Delta \mathbf{U}$, sendo possível atualizar o vetor solução da seguinte forma:

$$
\mathbf{U}_{k+1}^{n+1}=\mathbf{U}_{k}^{n}+\Delta \mathbf{U}
$$

em que $k$ representa o contador das iterações realizadas.

Este procedimento é repetido até que a variação no vetor solução seja muito pequena, isto é $\|\Delta \mathbf{U}\| \leq$ tolerância. Uma vez atingida esta condição, as acelerações são atualizadas: 


$$
\dot{\mathbf{U}}^{n+1}=\frac{1}{\Delta t}\left(\mathbf{U}^{n+1}-\mathbf{U}^{n}\right)
$$

e o processo é reiniciado para um novo passo de tempo utilizando-se a correção $\mathbf{U}_{p}^{n+1}$ como chute inicial de $\mathbf{U}^{n+1}$ :

$$
\mathbf{U}_{p}^{n+1}=\mathbf{U}^{n}+\Delta t \dot{\mathbf{U}}^{n}
$$

\subsubsection{Integrador de segunda ordem}

Para buscar uma solução ainda mais precisa, recorre-se a um integrador implícito de segunda ordem, que consiste na utilização da Regra do Trapézio, com preditor de Adams-Bashfort $(\mathrm{ABP})$, cuja relação geral é definida por:

$$
\mathbf{U}^{n+1}(t)=\mathbf{U}^{n}(t)+\frac{\Delta t}{2}\left[\alpha\left(t^{n+1}, \mathbf{U}^{n+1}\right)+\alpha\left(t^{n}, \mathbf{U}^{n}\right)\right]
$$

novamente para um problema de valor inicial do tipo:

$$
\alpha(t, \mathbf{U}(t))=\dot{\mathbf{U}}(t), \mathbf{U}\left(t_{0}\right)=\mathbf{U}_{0},
$$

Analogamente ao caso do integrador de primeira ordem, para um passo de tempo constante, ao substituir 4.30 em 4.43, obtém-se:

$$
\mathbf{U}^{n+1}=\mathbf{U}^{n}+\frac{\Delta t}{2} \overline{\mathbf{M}}^{-1}\left[\overline{\mathbf{F}}\left(\mathbf{U}^{n+1}\right)-\overline{\mathbf{K}}\left(\mathbf{U}^{n+1}\right)+\dot{\mathbf{U}}^{n}\right],
$$

ou ainda,

$$
\left[\frac{2}{\Delta t} \overline{\mathbf{M}}+\overline{\mathbf{K}}\left(\mathbf{U}^{n+1}\right)\right] \mathbf{U}^{n+1}=\frac{2}{\Delta t} \overline{\mathbf{M}} \mathbf{U}^{n}+\overline{\mathbf{M}} \dot{\mathbf{U}}^{n}+\overline{\mathbf{F}}\left(\mathbf{U}^{n+1}\right),
$$

onde novamente $\mathbf{U}^{n}$ e $\mathbf{U}^{n+1}$ são, respectivamente, os vetores incógnita nos tempos anterior e atual.

Aplicando-se o método de Newton-Raphson:

$$
\left[\frac{2}{\Delta t} \overline{\mathbf{M}}+\overline{\mathbf{K}}\left(\mathbf{U}^{n+1}\right)\right] \mathbf{U}^{n+1}-\frac{2}{\Delta t} \overline{\mathbf{M}} \mathbf{U}^{n}-\overline{\mathbf{M}} \dot{\mathbf{U}}^{n}-\overline{\mathbf{F}}\left(\mathbf{U}^{n+1}\right)=\mathbf{R}^{n+1},
$$

e procedendo de forma equivalente ao integrador de primeira ordem, expandindo $\mathbf{R}^{n+1}$ por série de Taylor, truncando-a novamente nos termos de segunda ordem e, por fim, calculando o operador tangente $\frac{\partial \mathbf{R}^{n}}{\partial \mathbf{U}^{n}}$, assim como $\mathbf{R}^{n}$, obtém-se:

$$
\begin{aligned}
{\left[\frac{2}{\Delta t} \overline{\mathbf{M}}+\overline{\mathbf{K}}\left(\mathbf{U}^{n}\right)+\frac{\partial \overline{\mathbf{K}}\left(\mathbf{U}^{n}\right)}{\partial \mathbf{U}^{n}}\right] \Delta \mathbf{U}=\overline{\mathbf{F}}\left(\mathbf{U}^{n}\right) } & -\overline{\mathbf{K}}\left(\mathbf{U}^{n}\right) \mathbf{U}^{n} \\
& +\overline{\mathbf{M}} \dot{\mathbf{U}}^{n-1}-\frac{2}{\Delta t} \overline{\mathbf{M}}\left[\mathbf{U}^{n}-\mathbf{U}^{n-1}\right]
\end{aligned}
$$

Da mesma forma, para um determinado passo de tempo $\mathbf{U}^{n}$, calcula-se $\Delta \mathbf{U}$ a partir da Eq. 4.48, obtendo-se $\mathbf{U}^{n+1}$ de acordo com a Eq. 4.40. Esse procedimento é repetido até que a 
condição $\|\Delta \mathbf{U}\| \leq$ tolerância seja satisfeita.

Para que um novo passo de tempo seja calculado, a aceleração é atualizada por meio de:

$$
\dot{\mathbf{U}}^{n+1}=\frac{2}{\Delta t}\left(\mathbf{U}^{n+1}-\mathbf{U}^{n}\right)-\dot{\mathbf{U}}^{n},
$$

e, analogamente ao caso anterior, utiliza-se o uma correção no vetor incógnita, neste caso o corretor de Adams-Bashfort, para o início do processo iterativo:

$$
\mathbf{U}_{p}^{n+1}=\mathbf{U}^{n}+\frac{\Delta t}{2}\left(\dot{\mathbf{U}}^{n}-\dot{\mathbf{U}}^{n-1}\right) .
$$

\subsubsection{Procedimento para o avanço temporal da solução}

Para realizar o avanço da solução de um tempo $t_{n}$ para o tempo $t_{n+1}$ seguem-se os passos listados a seguir, também ilustrados na Fig. 4.3:

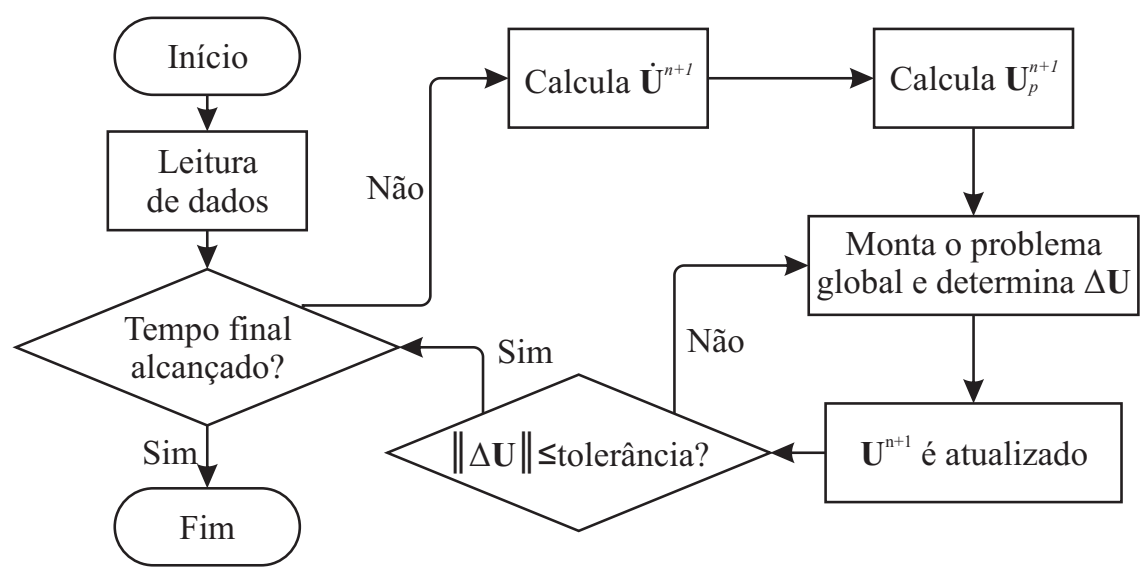

Figura 4.3: Fluxograma do procedimento de avanço temporal da solução.

1. Os valores de aceleração nodais são atualizados de acordo com as Eq. 4.41 ou 4.49;

2. O vetor tentativa, ou chute da solução é calculado através das Eq. 4.42 ou 4.50, destacandose que as variáveis de pressão não estão inclusas neste cálculo;

3. A solução real $\mathbf{U}^{n+1}$ é calculada com base nas Eq. 4.35 ou 4.46 por meio do método de Newton-Raphson;

4. Um novo passo de tempo é computado e o procedimento é repetido até que se alcance o tempo final da análise.

\subsection{Exemplos de problemas de dinâmica dos fluidos}

Esta seção é dedicada ao teste da formulação numérica desenvolvida anteriormente por meio de aplicações numéricas que possibilitem a verificação e validação de seus resultados. 
Apesar de o código desenvolvido permitir a análise de escoamentos tridimensionais, são estudados apenas casos bidimensionais por serem exemplos confiáveis disponíveis na literatura. Os exemplos escolhidos são amplamente utilizados na verificação e validação de metodologias para análise de escoamentos incompressíveis além de demandarem menor custo computacional por se tratarem de casos bidimensionais.

No primeiro deles, o caso clássico de uma cavidade quadrada com parede móvel é simulado. Em seguida, testa-se o rompimento de uma barragem, em que se faz necessária a utilização do esquema de movimentação do domínio pela descrição ALE. Por fim, estudou-se o escoamento bidimensional sobre um cilindro.

\subsubsection{Cavidade quadrada}

Neste primeiro caso, estudou-se o comportamento de um fluido confinado em uma cavidade cujo escoamento é induzido pelo deslizamento da parede superior, como ilustra a Fig. 4.4. As paredes laterais da cavidade, por sua vez, são rígidas e com condição de nãoescorregamento. Já nas faces perpendiculares ao eixo $z$, prescreve-se apenas que a componente de velocidade normal ao plano (w) é nula.

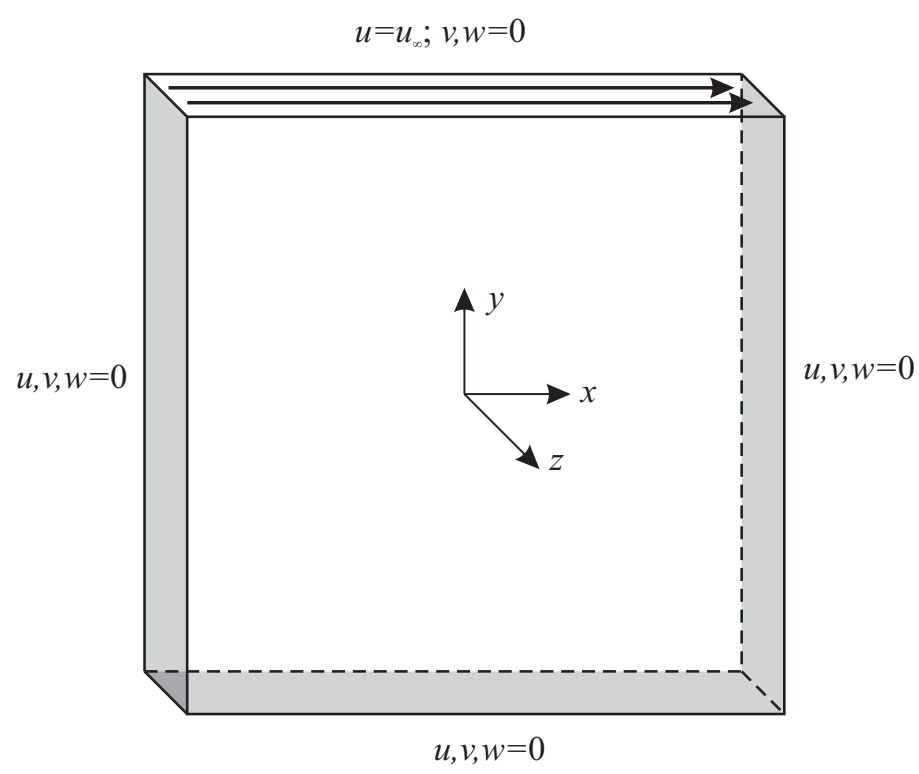

Figura 4.4: Geometria e condições de contorno do problema da cavidade quadrada.

Devido à viscosidade do fluido, o deslizamento da parede localizada no topo da cavidade provoca a formação de vórtices no interior da cavidade. À medida em que o valor de $u_{\infty}$ é variado, altera-se o número de Reynolds (Eq. 4.51) do problema (para viscosidade, massa específica e geometria constantes):

$$
R e=\frac{\rho L u_{\infty}}{\mu}=\frac{L u_{\infty}}{v}
$$


em que $v$ é a viscosidade cinemática do fluido, $u_{\infty}$ a velocidade de referência e $L$ é chamado de comprimento característico do escoamento, neste caso igual ao comprimento ou a altura da cavidade.

Neste trabalho foram testadas três diferentes malhas, ilustradas na Fig. 4.5 e cujos dados são apresentados na Tabela 4.1.

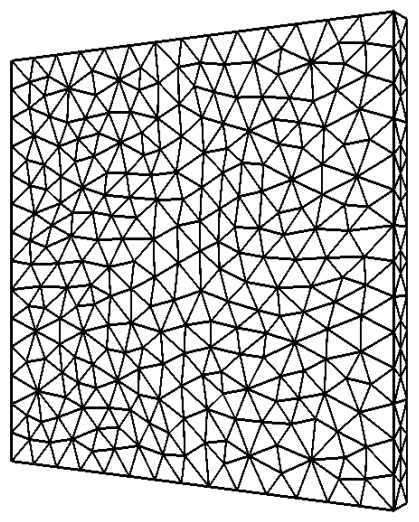

(a)

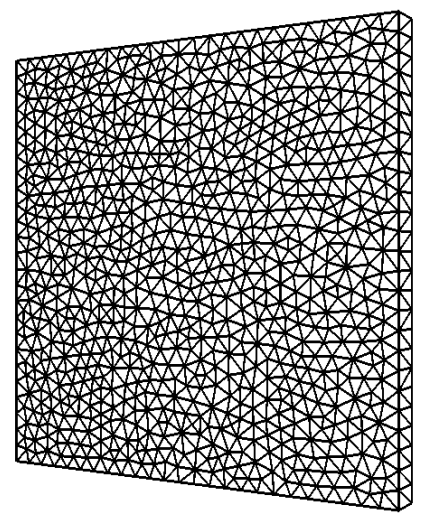

(b)

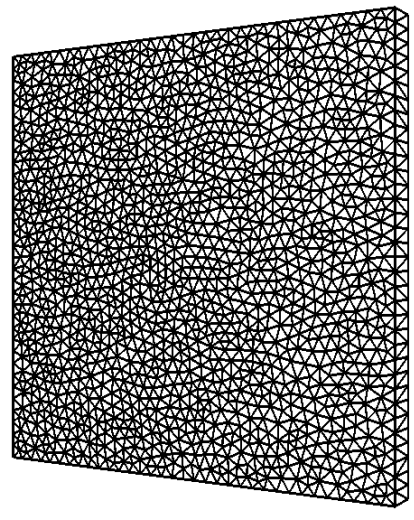

(c)

Figura 4.5: Malhas de elementos finitos utilizadas: (a) malha 1, (b) malha 2 e (c) malha 3.

Tabela 4.1: Características das malhas utilizadas no problema da cavidade quadrada

\begin{tabular}{ccccc}
\hline Malha & Elementos & Nós - Pressão & Nós - Velocidade & Graus de liberdade \\
\hline Malha 1 & 1844 & 632 & 3737 & 11843 \\
Malha 2 & 5085 & 1791 & 10446 & 33129 \\
Malha 3 & 9034 & 3140 & 18437 & 58451 \\
\hline
\end{tabular}

A análise desse problema consiste em obter o estado estacionário, isto é, a análise é levada até o momento em que o campo de velocidades e de pressões se torne constante. Como parâmetros de entrada, utilizaram-se valores unitários de $\rho$ e $\mu$, variando-se o módulo de $u_{\infty}$. Para todas as análises empregaram-se passos de tempo que variam de 1,00 a 0,01, com valores menores para os casos com maior Re.

A seguir, são apresentados os resultados obtidos para quatro diferentes números de Reynolds: 100, 400, 1000 e 3200. Tendo em vista que a origem do sistema de referência encontrase no centro da cavidade e que esta possui lado igual a duas unidades, apresentam-se na Fig. 4.6 as curvas da velocidades horizontal e vertical adimensionalizadas $\left(u / u_{\infty}\right.$ e $\left.v / u_{\infty}\right)$ ao longo das linhas centrais da cavidade em comparação com as obtidas por Ghia, Ghia e Shin (1982).

Para valores superiores de $R e$ não obtiveram-se respostas estáveis. Ao explorar os gráficos da Fig. 4.6, pode-se atestar a convergência da metodologia empregada para a solução teórica à medida em que utiliza-se uma malha mais refinada. Contudo, mesmo para os casos com $R e=400$ 


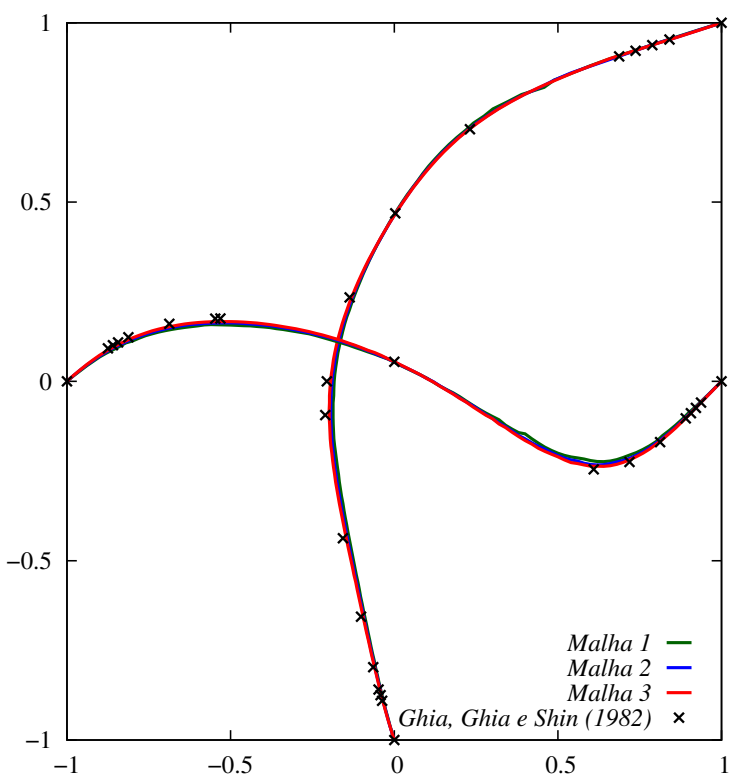

(a) $R e=100, \Delta t=1,00$.

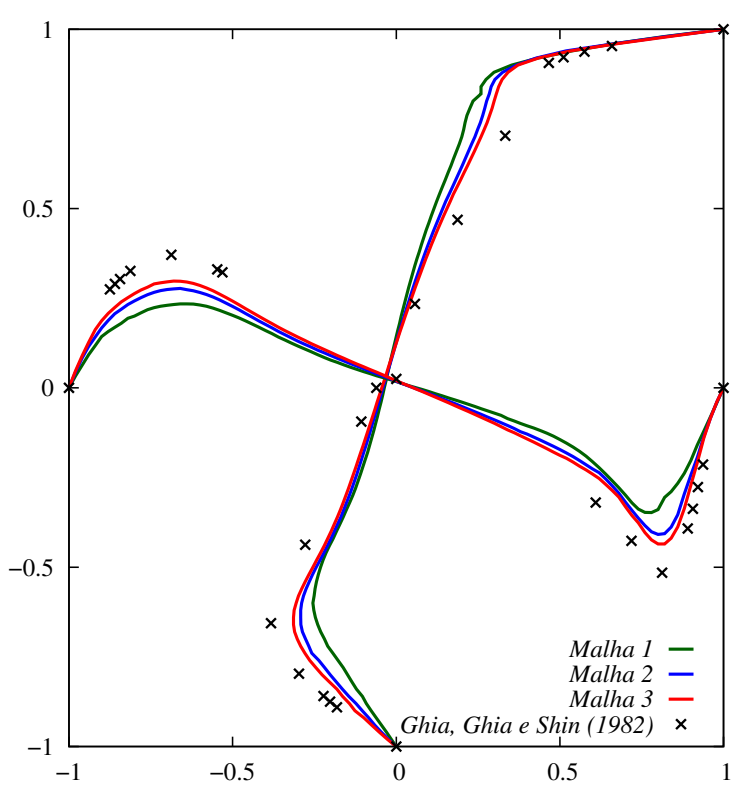

(c) $\operatorname{Re}=1000, \Delta t=0,10$.

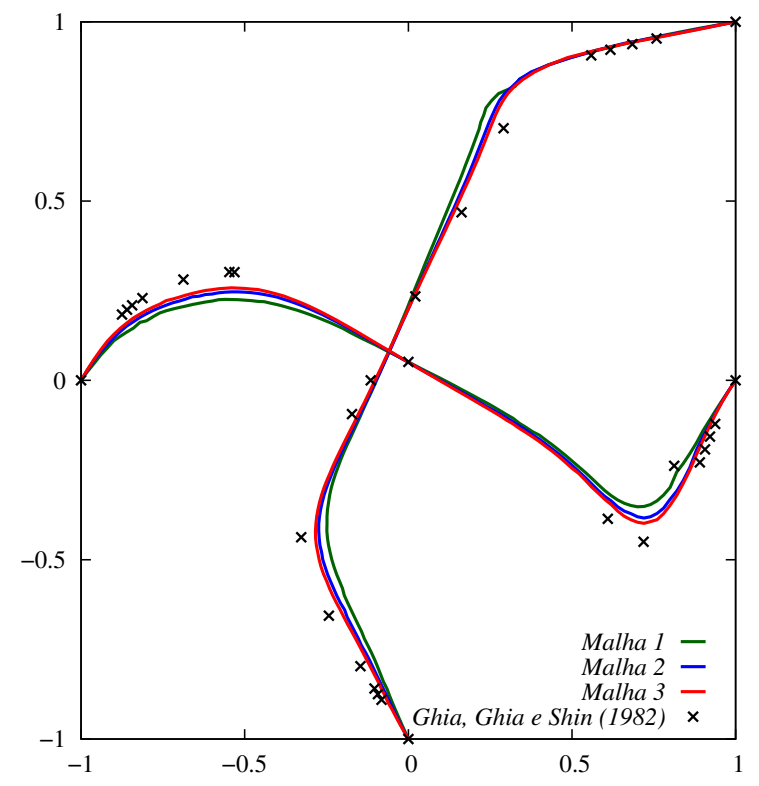

(b) $R e=400, \Delta t=0,10$.

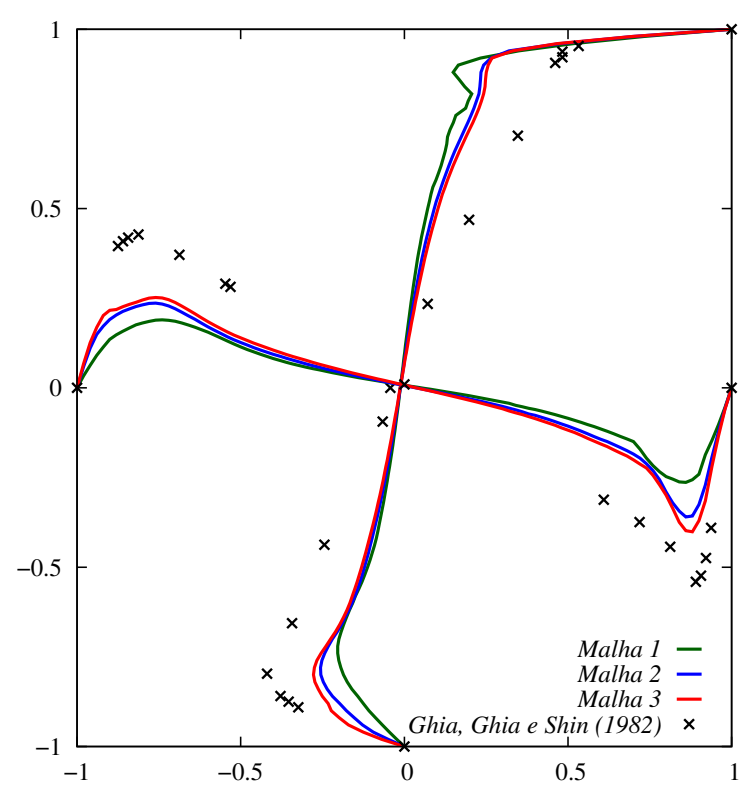

(d) $R e=3200, \Delta t=0,01$.

Figura 4.6: Velocidades adimensionais em função da altura/largura da cavidade.

e 1000 uma resposta mais satisfatória demanda num acréscimo muito grande no número de graus de liberdade do problema.

Isso ocorre porque à medida em que aumenta-se o número de Reynolds, maiores são os efeitos turbulentos associados ao problema. As equações de Navier-Stokes são capazes de representar satisfatoriamente estes efeitos, mas demandam de uma malha fina o suficiente para captar as diversas escalas de turbulência.

Outros meios de se alcançar soluções com certa ordem de precisão sem estender o número de graus de liberdade do sistema são pelo emprego de modelos de turbulência, que não 
fazem parte dos objetivos deste trabalho.

Por fim, apresentam-se nas Fig. 4.7 e 4.8 a comparação entre as linhas de corrente e de pressão obtidas para a malha 3 em comparação com as de Glowinski (2003), onde é possível verificar boa aproximação dos resultados.

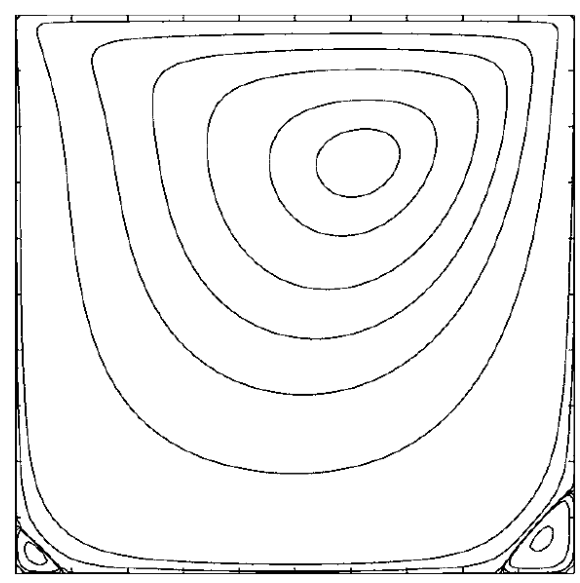

(a) Glowinski (2003), $R e=100$

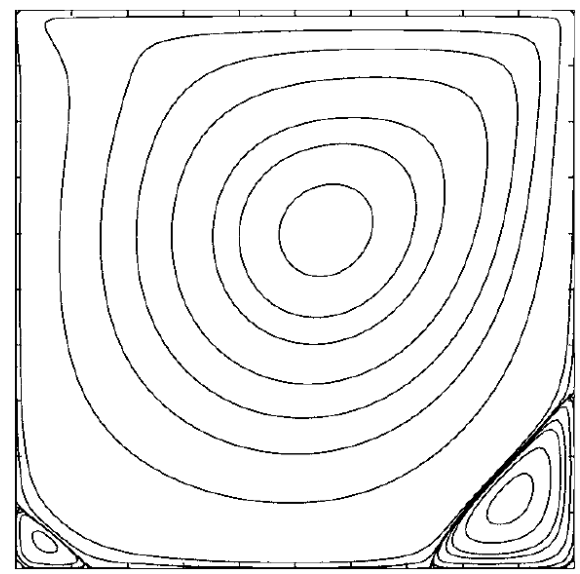

(c) Glowinski (2003), $R e=400$

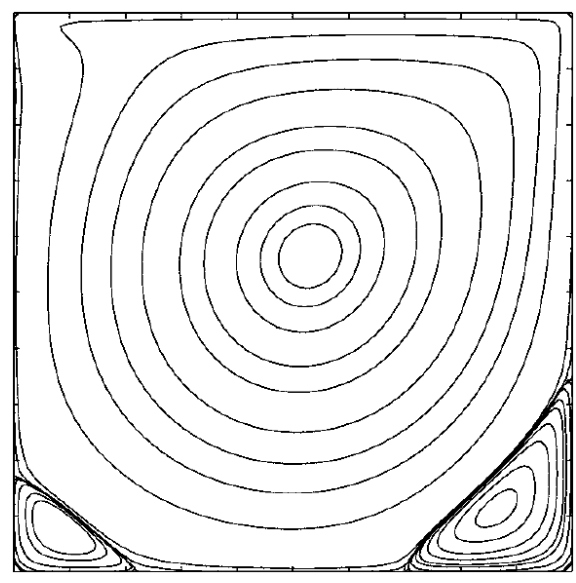

(e) Glowinski (2003), $R e=1000$

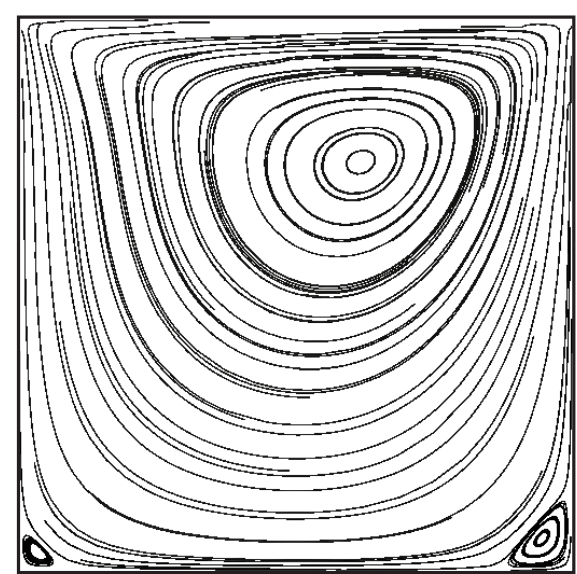

(b) Presente trabalho, $R e=100$

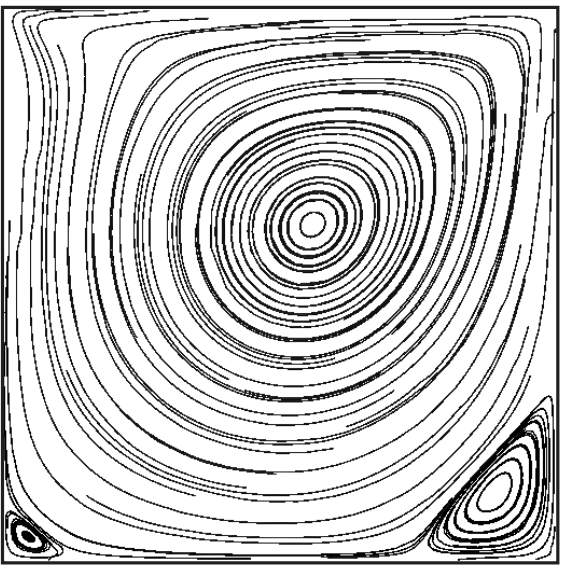

(d) Presente trabalho, $R e=400$

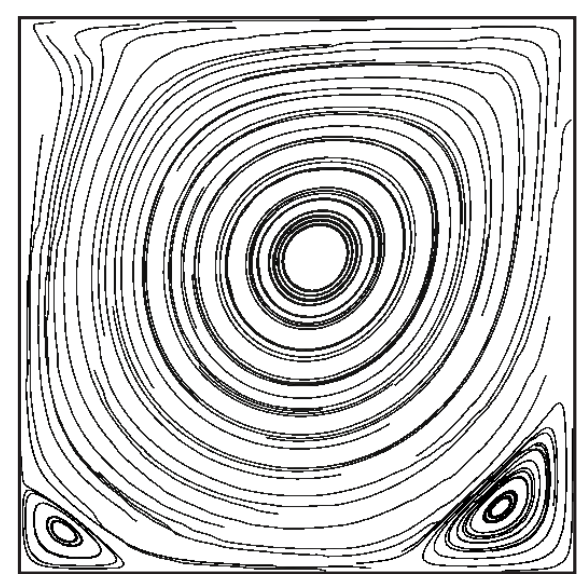

(f) Presente trabalho, $R e=1000$

Figura 4.7: Linhas de corrente obtidas por Glowinski (2003) (à esquerda) e no presente trabalho. 


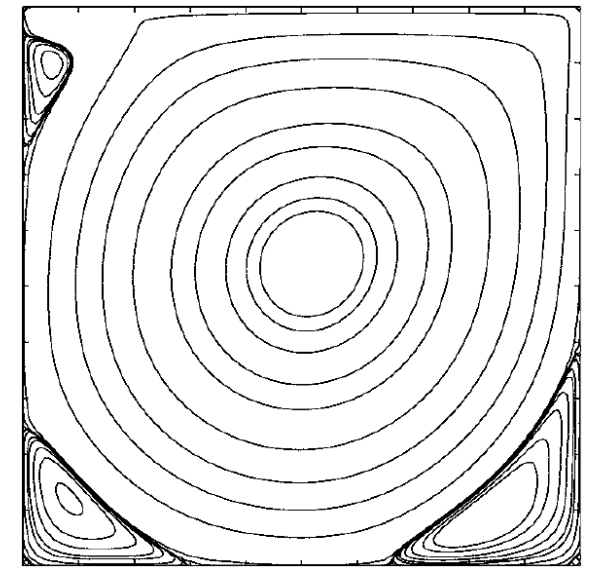

(g) Glowinski (2003), $R e=3200$

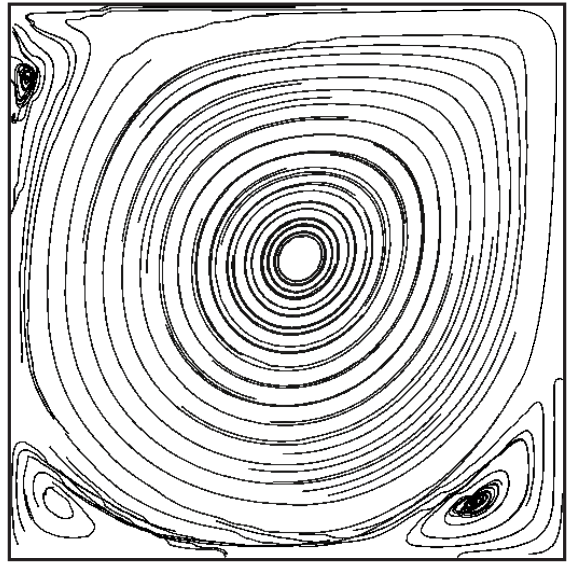

(h) Presente trabalho, $R e=3200$

Figura 4.7: Linhas de corrente obtidas por Glowinski (2003) (à esquerda) e no presente trabalho - continuação.

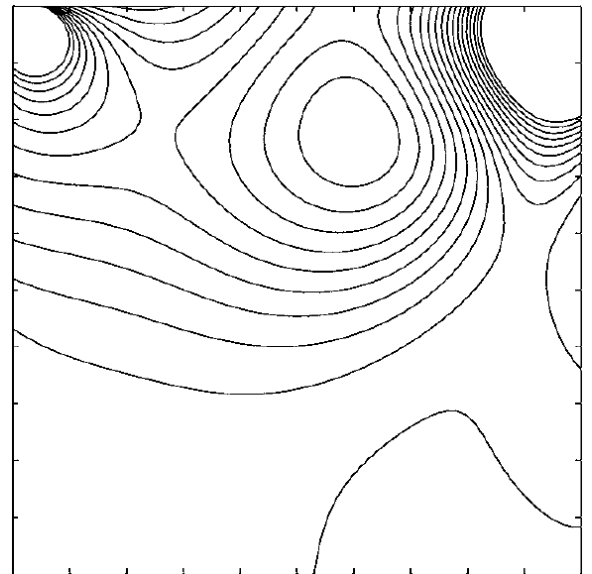

(a) Glowinski (2003), $R e=100$

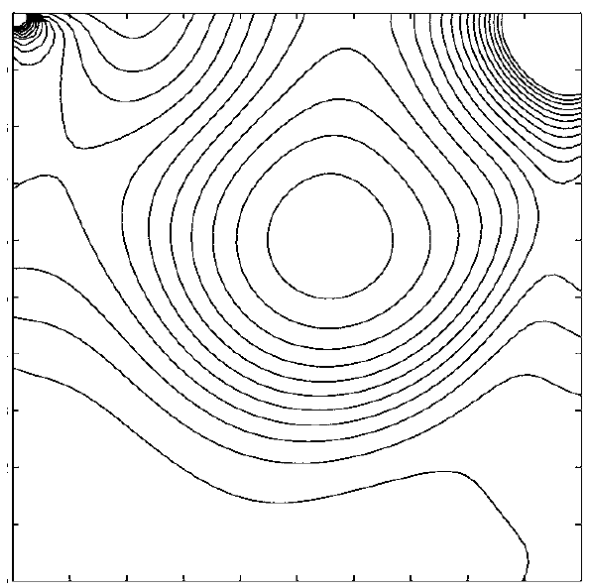

(c) Glowinski (2003), $R e=400$

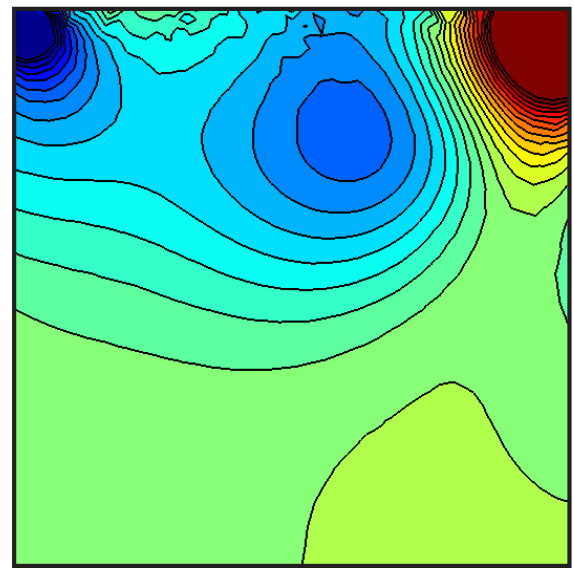

(b) Presente trabalho, $R e=100$

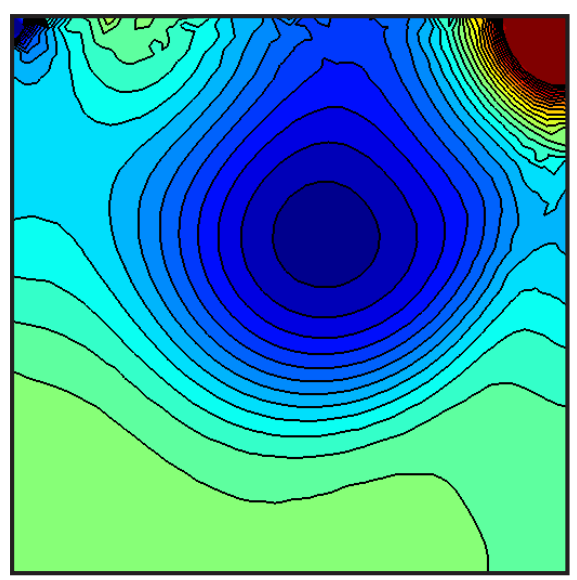

(d) Presente trabalho, $R e=400$

Figura 4.8: Linhas de pressão obtidas por Glowinski (2003) (à esquerda) e no presente trabalho. 


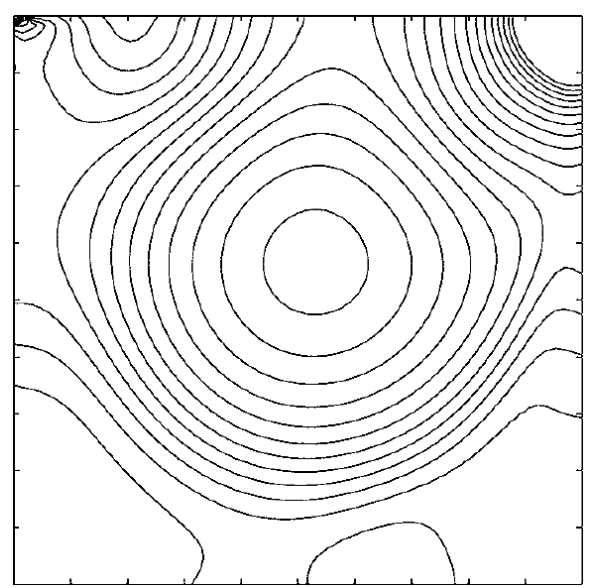

(e) Glowinski (2003), $R e=1000$

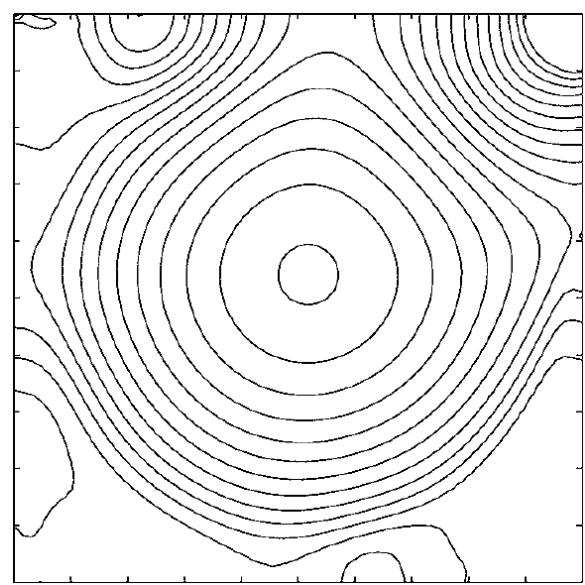

(g) Glowinski (2003), $R e=3200$

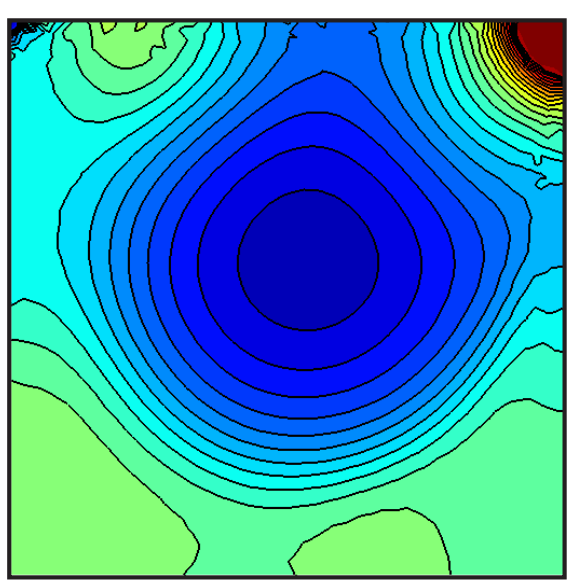

(f) Presente trabalho, $R e=1000$

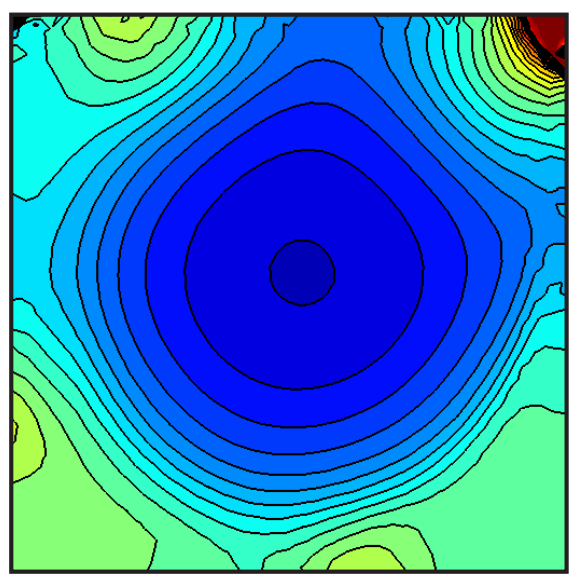

(h) Presente trabalho, $R e=3200$

Figura 4.8: Linhas de pressão obtidas por Glowinski (2003) (à esquerda) e no presente trabalho continuação.

\subsubsection{Barragem rompida}

Neste segundo exemplo considerado, simulou-se o problema de uma barragem inicialmente em repouso cuja comporta se rompe no instante inicial da análise, permitindo o escoamento do fluido. Escolheu-se realizar a simulação fazendo $u_{i}=\omega_{i}$, ou seja, uma descrição puramente Lagrangeana. Assim, as posições de cada nó da malha utilizada para a discretização espacial são atualizadas com base nos próprios valores nodais da velocidade do fluido. É importante destacar que nenhum método de suavização ou movimentação da malha a fim de evitar a distorção dos elementos é aplicado. Tal assunto será abordado com maior detalhe mais adiante, no Capítulo 6.

Na Fig. 4.9 a geometria bem como as condições de contorno do problema são ilustradas. Como o fluido encontra-se inicialmente em repouso e sob ação da força gravitacional $g$, sua distribuição de pressão é hidrostática. Além disso, o fato de as paredes serem lisas implica na restrição apenas da componente normal de velocidade nestes contornos. Novamente nas faces perpendiculares à direção $z$ restringe-se a componente normal de velocidades e consideram-se superfícies livres nos demais contornos. 

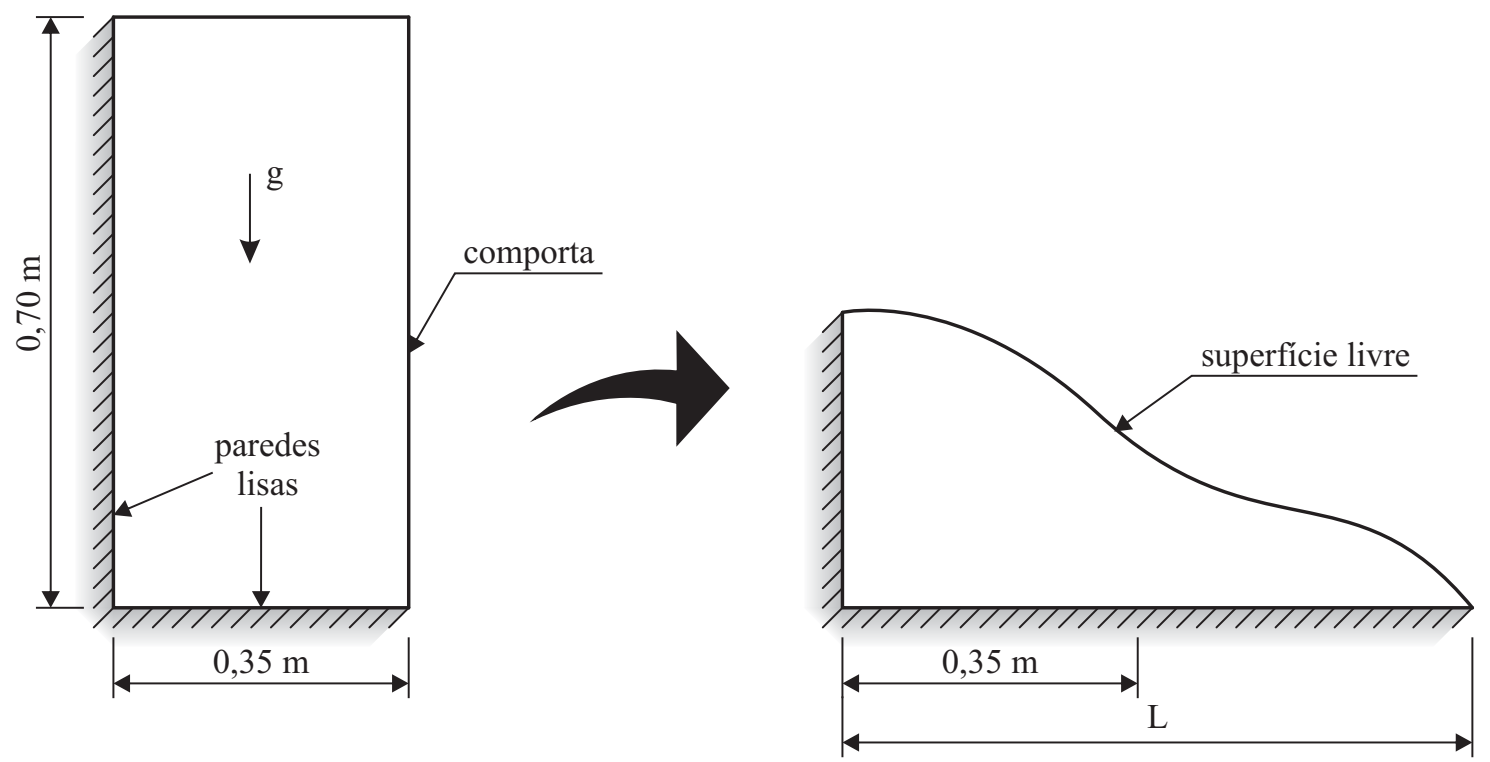

Figura 4.9: Geometria do problema da barragem rompida.

A malha utilizada para a simulação é ilustrada na Fig. 4.10 e conta com 2593 elementos, sendo que pressão e velocidade são interpoladas por 808 e 4961 nós, respectivamente, implicando num total de 15691 parâmetros nodais. No canto inferior direito da malha adotou-se uma maior densidade de elementos, por se tratar da região onde ocorre maior distorção.

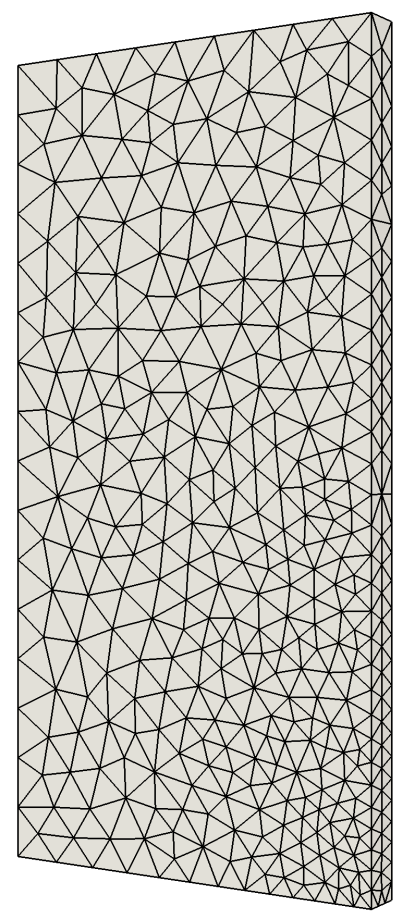

Figura 4.10: Malha utilizada na simulação do problema da barragem rompida.

Para o fluido, adotou-se massa específica igual a $1,0 \mathrm{~kg} / \mathrm{m}^{3}$, viscosidade dinâmica de $0,001 \mathrm{~Pa} . s$, aceleração da gravidade igual a $1,0 \mathrm{~m} / \mathrm{s}^{2}$ e $\Delta t=5 \cdot 10^{-4} \mathrm{~s}$. 
Na Fig. 4.11 apresenta-se a o gráfico obtido para o deslocamento máximo do fluido em função do tempo adimensional, comparando-se com os resultados experimentais de Martin e Moyce (1952) e numéricos de Nithiarasu (2005), que simulou o problema utilizando uma análise bidimensional com elementos finitos triangulares com base no algoritmo CBS (characteristicbased split). No gráfico, L é a posição extrema do fluido, $\mathrm{L}_{0}=0,35 \mathrm{~m}$ e t* é o tempo adimensional, tal que $\mathrm{t}^{*}=t \sqrt{2 g / \mathrm{L}_{0}}$.

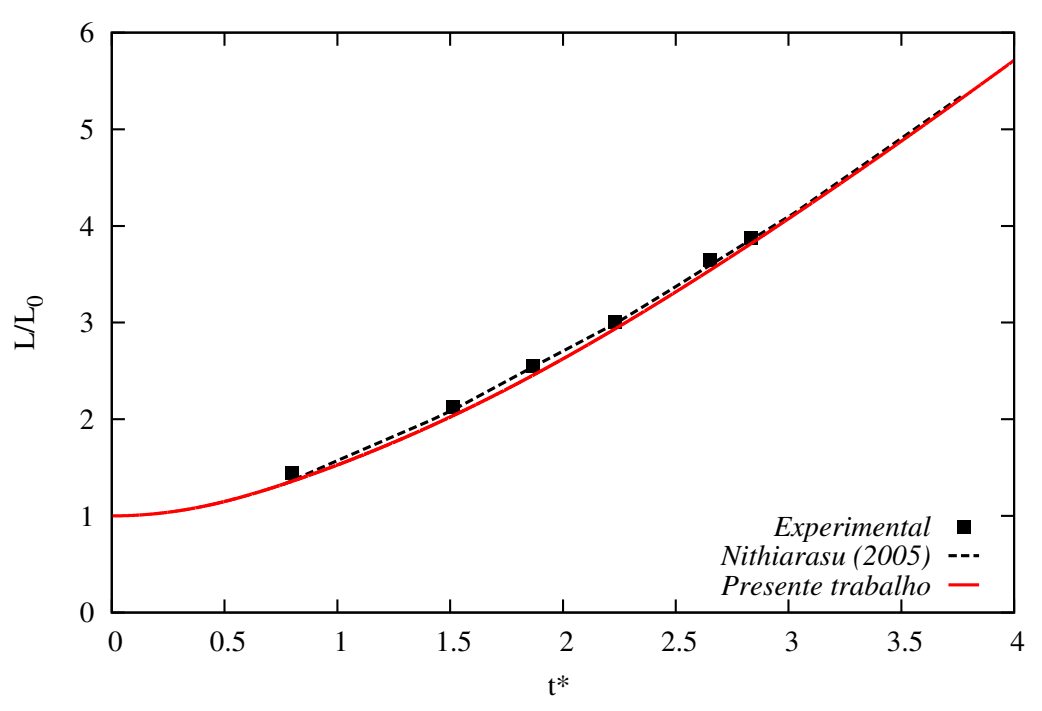

Figura 4.11: Deslocamento horizontal relativo em função do tempo adimensional.

Como pode-se perceber, os valores obtidos mostram concordância com as referências utilizadas. A simulação foi realizada utilizando tanto o integrador temporal de primeira ordem quanto o de segunda ordem, não sendo observadas diferenças significativas nos resultados.

Por fim, as Fig. 4.12, 4.13 e 4.14 apresentam as configurações deformadas da malha bem como os campos de velocidade e pressão nos instantes de tempo t* iguais a 1,5, 2,5 e 4,0, respectivamente. 


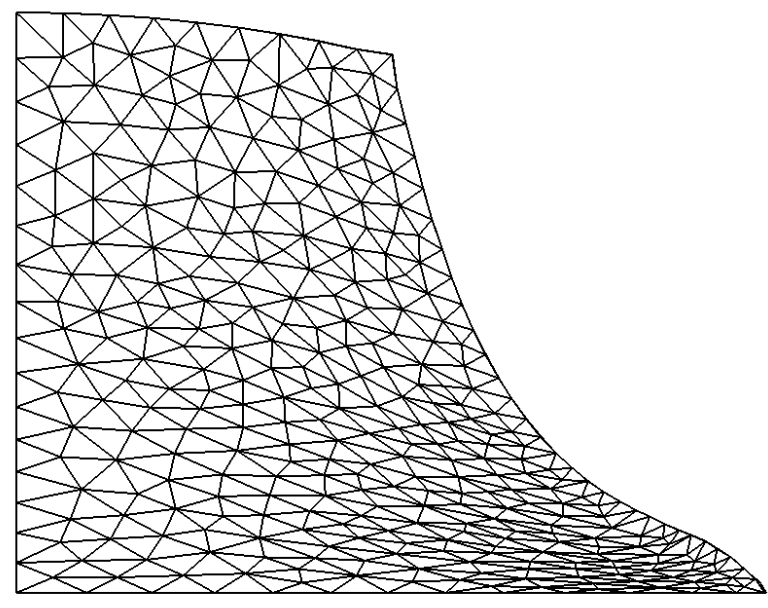

(a)

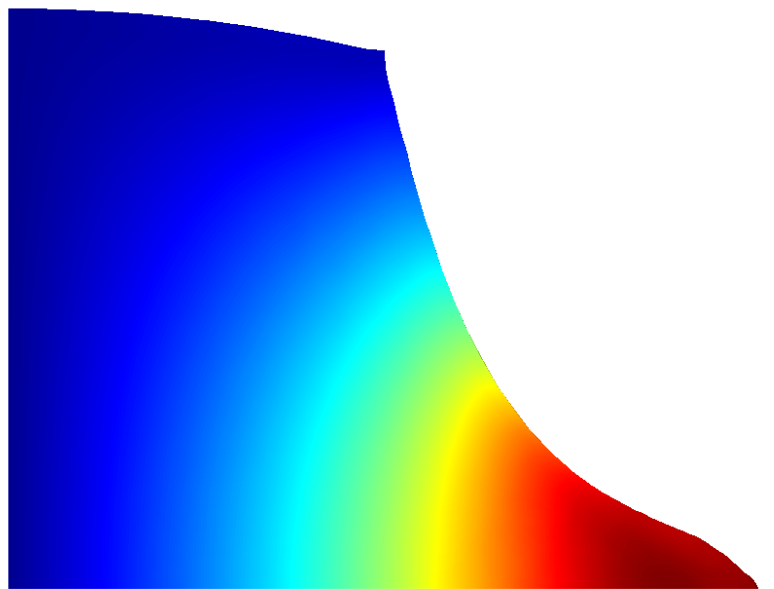

Velocidade $x$

0,2

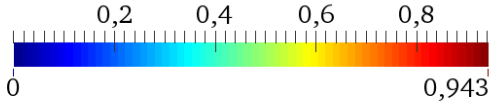

(c)

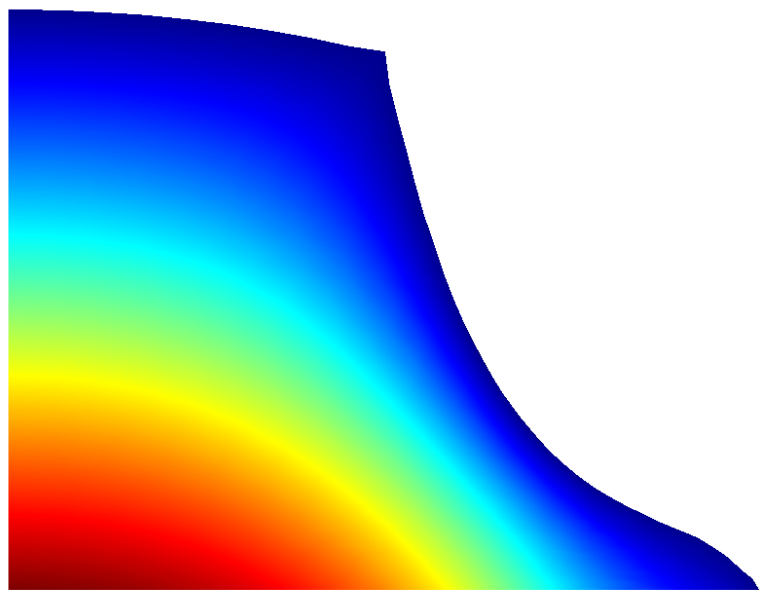

Pressão

0,2

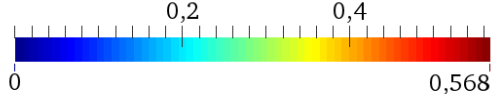

(b)

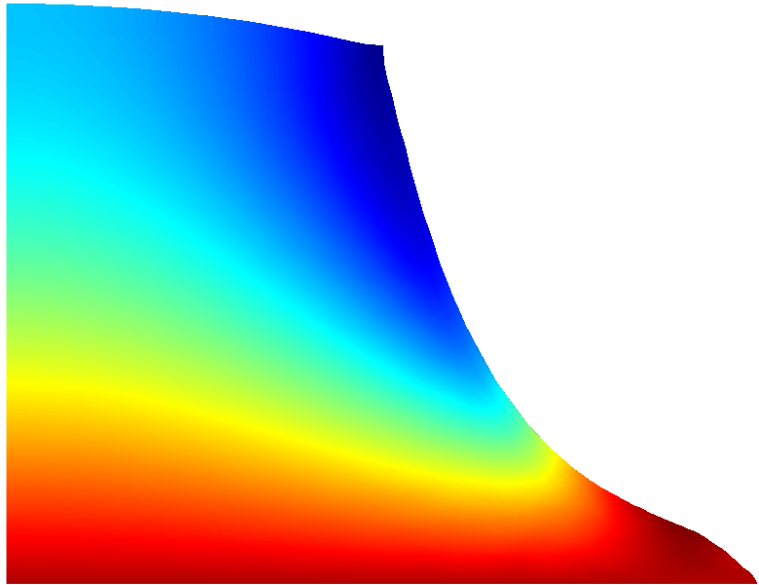

Velocidade y

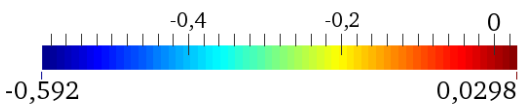

(d)

Figura 4.12: Instante de tempo $t^{*}=1,5:$ (a) malha deformada, (b) pressão, (c) velocidade $x$ e (d) velocidade $y$. 


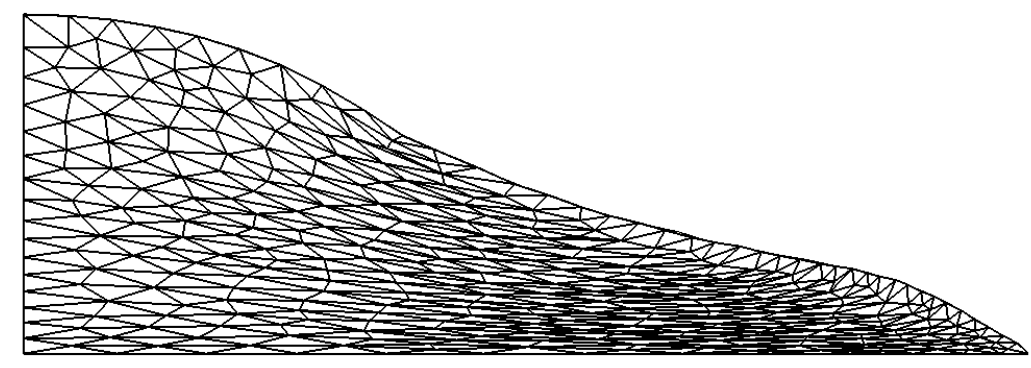

(a)
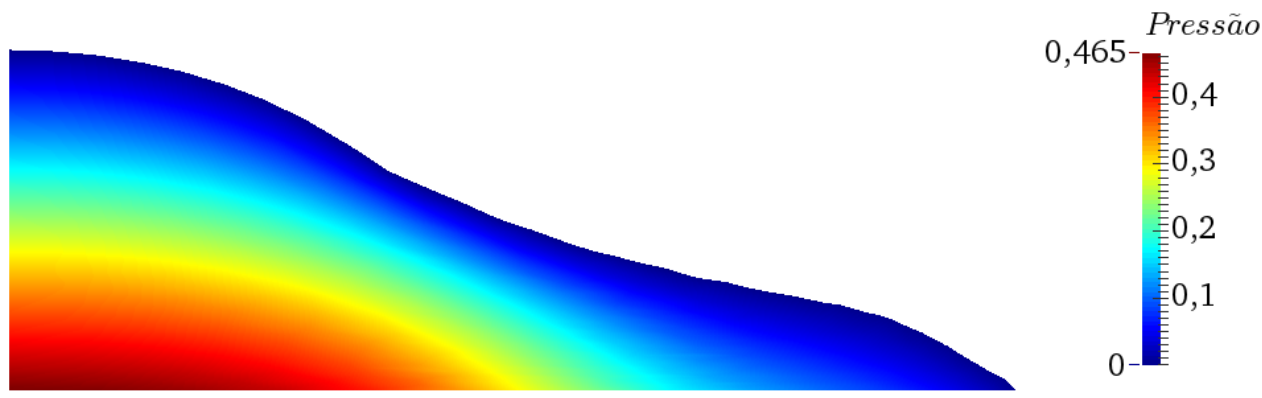

(b)
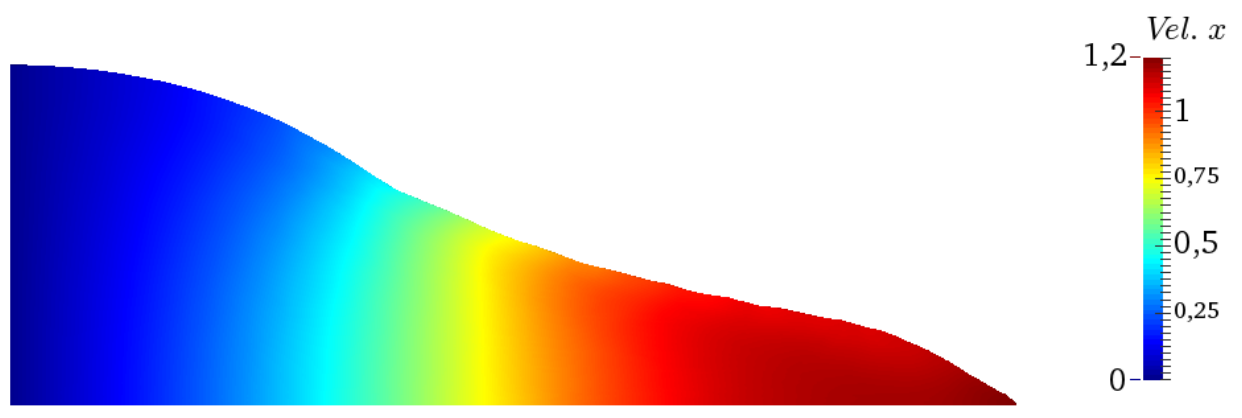

(c)

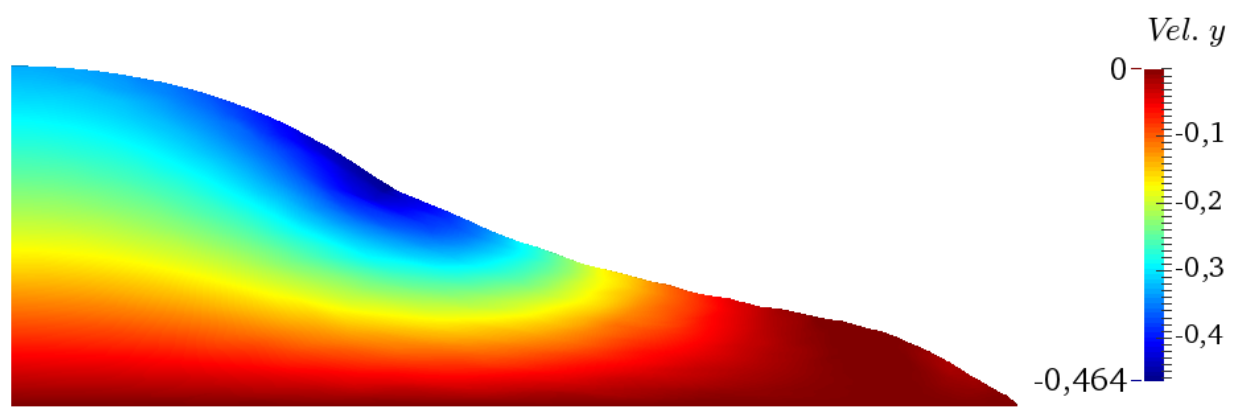

(d)

Figura 4.13: Instante de tempo $t^{*}=2,5$ : (a) malha deformada, (b) pressão, (c) velocidade $x$ e (d) velocidade $y$. 


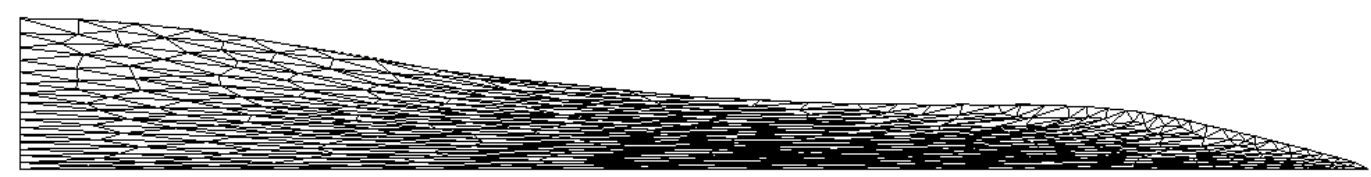

(a)

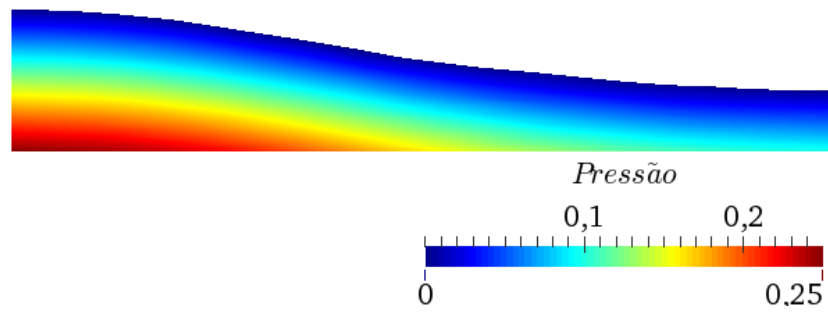

(b)

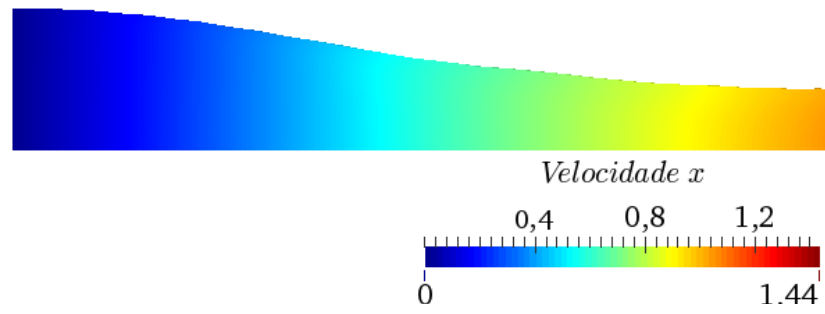

(c)

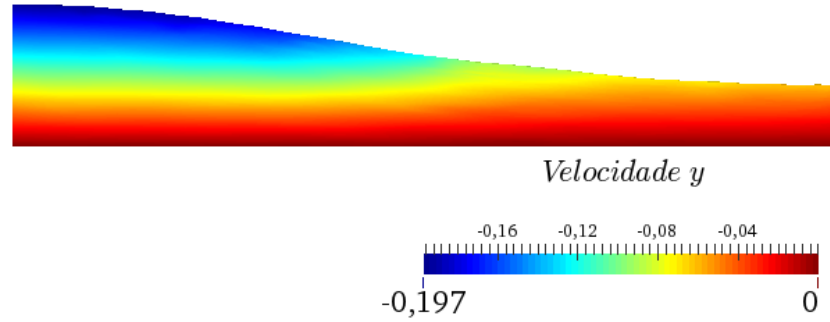

(d)

Figura 4.14: Instante de tempo $t^{*}=4,0$ : (a) malha deformada, (b) pressão, (c) velocidade $x$ e (d) velocidade $y$. 


\subsubsection{Escoamento em torno de um cilindro}

Nesta aplicação, tem-se por objetivo a comparação das respostas transientes e estacionárias obtidas em cada modelo de integração temporal proposto anteriormente. Trata-se do escoamento bidimensional sobre um cilindro, cuja geometria e condições de contorno são ilustradas na Fig. 4.15. Por se tratar de um escoamento viscoso, exceto para $R e \ll 1$, há a formação de vórtices à jusante do cilindro. Para valores de $\mathrm{Re} \lesssim 40$, observa-se a ocorrência de dois vórtices simétricos. Já para um intervalo da ordem de $40<\operatorname{Re}<200$, é gerada uma esteira de vórtices denominada esteira de Von Kármán. No entanto, nesse intervalo a esteira de vórtices é ainda laminar. Para valores de Re superiores a 200, ocorre uma zona de transição e em seguida a turbulência do escoamento.

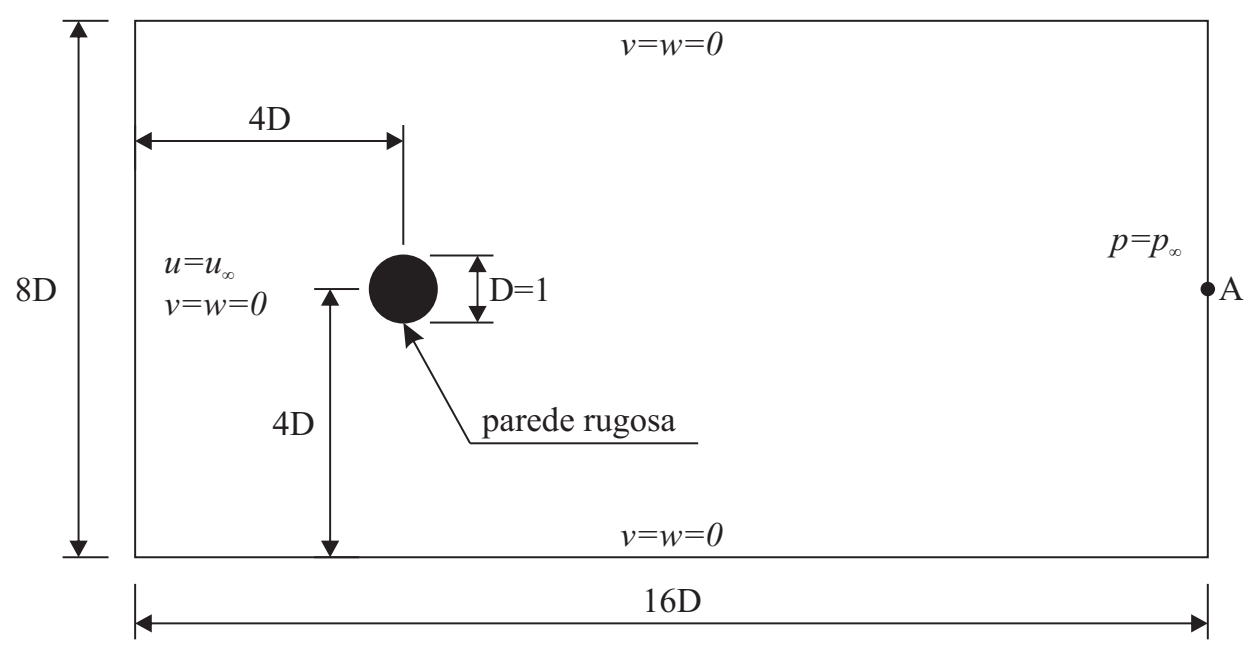

Figura 4.15: Geometria do problema de escoamento em torno de um cilindro.

Como não faz parte do escopo deste trabalho a avaliação de efeitos turbulentos, optou-se por simular este problema com $\operatorname{Re}=100$, obtidos fixando-se $\rho=u_{\infty}=1,0$ e $\mu=0,01$. O problema foi simulado para passos de tempo iguais a 0,1 e 0,05 com a malha apresentada na Fig. 4.16 (vista frontal), que conta com 5195 elementos e 10427 nós que interpolam o campo de velocidade, dos quais 1744 também aproximam o campo de pressão, num total de 33025 graus de liberdade.

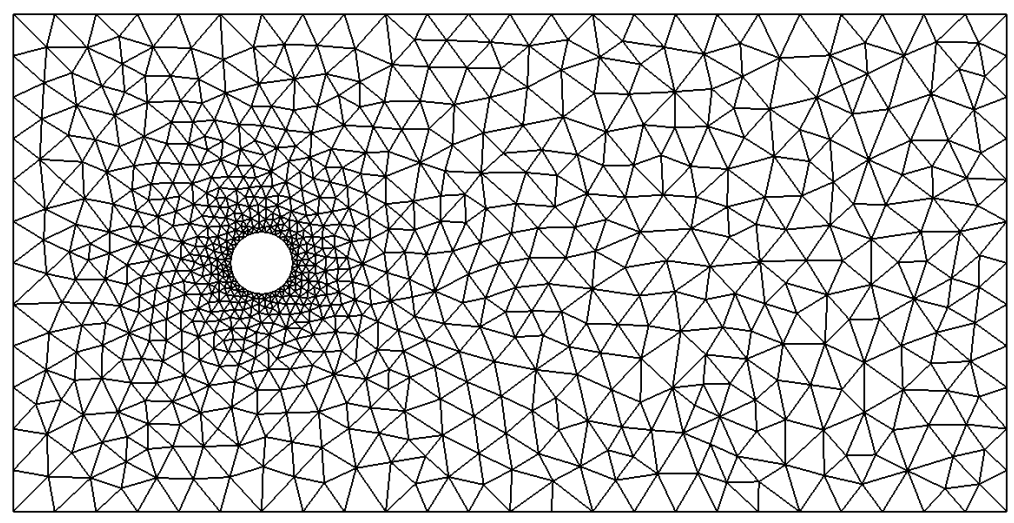

Figura 4.16: Malha utilizada nas simulações de escoamento em torno de um cilindro. 
O primeiro parâmetro avaliado neste exemplo foi a componente vertical de velocidade no ponto A (ver Fig. 4.15). Na Fig. 4.17 é apresentado sua evolução com o tempo utilizando-se o integrador temporal de segunda ordem com $\Delta t=0,05$ em comparação com o resultado reportado em Sampaio et al. (1993), verificando-se boa aproximação.

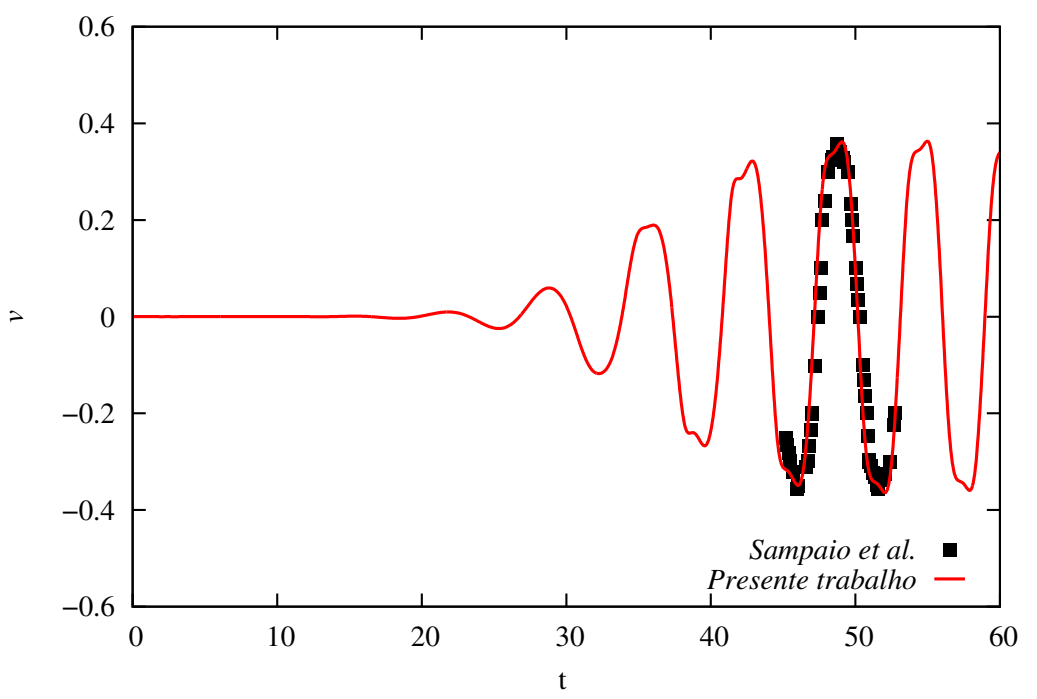

Figura 4.17: Componente vertical de velocidade no ponto A ao longo do tempo.

Para facilitar a comparação com trabalhos anteriores, calcularam-se também o número de Strouhal (St) e os coeficientes de sustentação $\left(\mathrm{C}_{L}\right)$ e arrasto $\left(\mathrm{C}_{D}\right)$ atuantes no cilindro:

$$
\begin{gathered}
\mathrm{St}=\frac{f D}{u_{\infty}}, \\
\mathrm{C}_{L}=\frac{F_{L}}{\frac{1}{2} \rho u_{\infty}^{2} t}, \\
\mathrm{C}_{D}=\frac{F_{D}}{\frac{1}{2} \rho u_{\infty}^{2} t},
\end{gathered}
$$

em que $f$ é a frequência de desprendimento dos vórtices, $t$ é a espessura do cilindro e $F_{L}$ e $F_{D}$ são, respectivamente, as forças de sustentação e arrasto calculadas por:

$$
\begin{aligned}
& F_{L}=\int_{S}\left(-p \delta_{1 j}+\tau_{1 j}\right) n_{j} d S, \\
& F_{D}=\int_{S}\left(-p \delta_{2 j}+\tau_{2 j}\right) n_{j} d S,
\end{aligned}
$$

em que $S$ representa a superfície do cilindro e $n_{j}$ a componente na direção $j$ do vetor normal à $S$ e 1 e 2 indicam, respectivamente, as direções horizontal e vertical.

Desse modo, nas Fig. 4.18 e 4.19 são apresentados os comportamentos de $C_{D}$ e $C_{L}$ observados para $\Delta t=0,1$ e $\Delta t=0,05$, respectivamente para ambos os integradores temporais.

Pode-se constatar uma grande diferença nos resultados obtidos, principalmente no que diz respeito à amplitude e frequência de oscilação do coeficiente de sustentação e também no valor médio do coeficiente de arrasto, mas que tendem a se aproximar à medida em que o passo 
de tempo é reduzido. Para avaliar melhor tais resultados, construiu-se também a Tabela 4.2, apresentada a seguir.

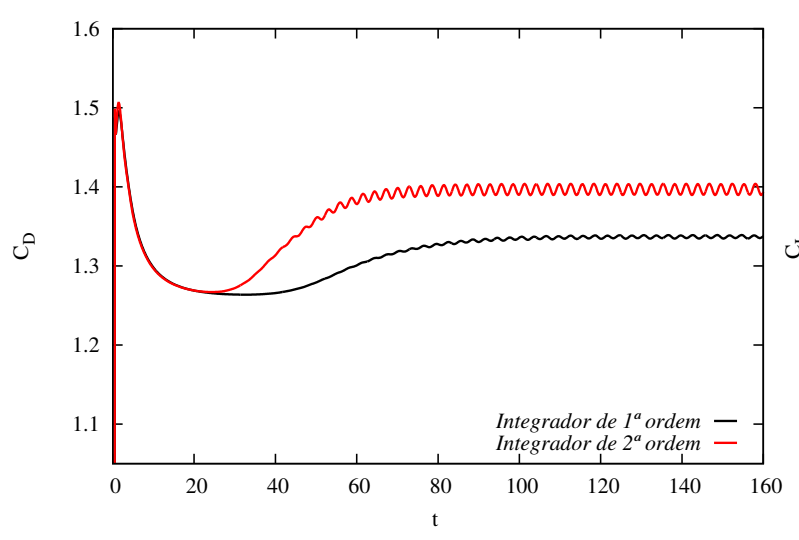

(a)

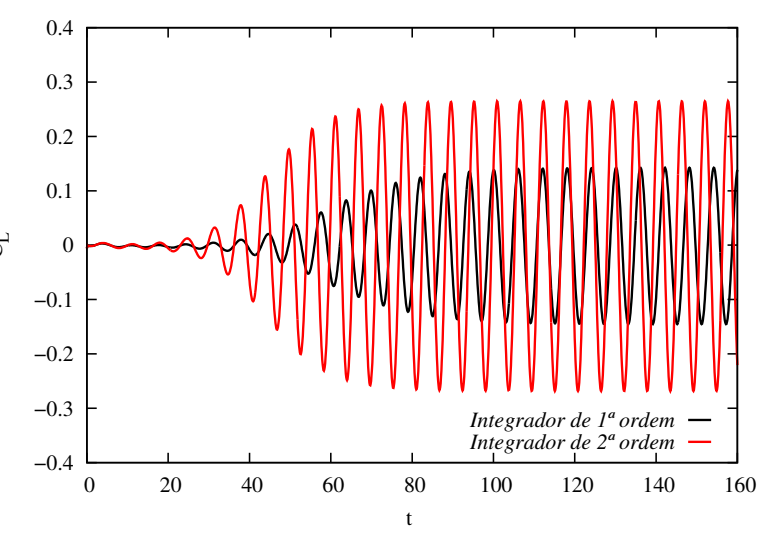

(b)

Figura 4.18: Coeficiente de (a) arrasto e (b) sustentação ao longo do tempo para $\Delta t=0,1$.

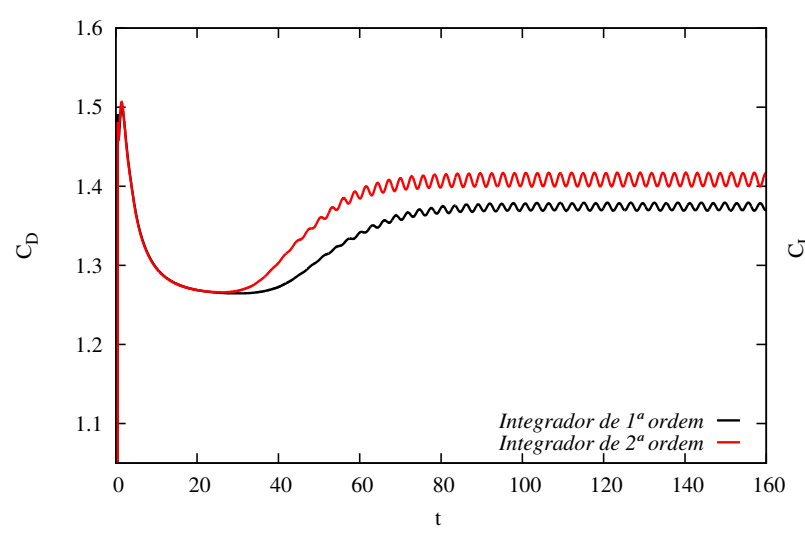

(a)

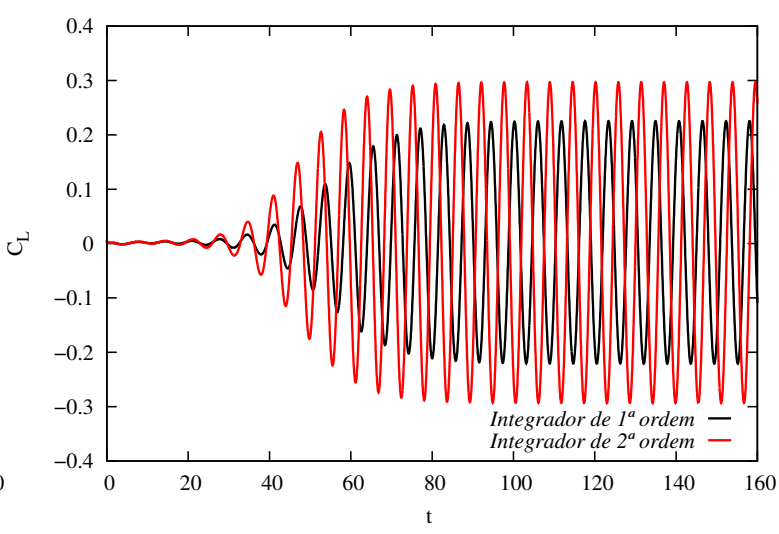

(b)

Figura 4.19: Coeficiente de (a) arrasto e (b) sustentação ao longo do tempo para $\Delta t=0,05$.

Diante dos dados apresentados, excetuando-se o caso do integrador de primeira ordem com $\Delta t=0,1$, principalmente devido aos valores do coeficiente de sustentação, os demais testes aproximam-se daqueles reportados na literatura. Comparando os integradores temporais, observase uma maior proximidade com os resultados presentes na literatura para o integrador de segunda ordem, o que se mostra coerente, uma vez que este possui uma ordem de aproximação maior.

Entretanto, apesar de o integrador de primeira ordem levar um tempo maior para atingir o estado estacionário, apresenta resultados satisfatórios para $\Delta t=0,05$ tanto para os coeficientes de arrasto e sustentação quanto para o valor de $S t$, quando comparados por exemplo com os valores obtidos por Ding et al. (2004).

É importante destacar que a escolha do passo de tempo tem influência considerável na resposta obtida. É notável também que a não-linearidade do problema torna a escolha de sua magnitude parte de vital importância da etapa de pré-processamento. 
Tabela 4.2: Comparativo entre os valores de $C_{D, \text { médio }}, C_{L, \text { máximo }}$ e St.

\begin{tabular}{lccc}
\hline Trabalho & $C_{D, \text { médio }}$ & $C_{L, \text { máximo }}$ & $S t$ \\
\hline Presente $-\Delta t=0,1$, int. $1^{\mathrm{a}}$ ordem & 1,32 & 0,14 & 0,167 \\
Presente $-\Delta t=0,1$, int. $2^{\mathrm{a}}$ ordem & 1,37 & 0,26 & 0,175 \\
Presente $-\Delta t=0,05$, int. $^{\mathrm{a}}$ ordem & 1,36 & 0,23 & 0,175 \\
Presente $-\Delta t=0,05$, int. 2 ${ }^{\mathrm{a}}$ ordem & 1,39 & 0,30 & 0,180 \\
Najafi, Arefmanesh e Enjilela (2012) & 1,47 & 0,38 & - \\
Ding et al. (2004) & 1,33 & 0,28 & 0,164 \\
Kjellgren (1997) & $1,34-1,37$ & $0,25-0,29-0,30-0,33$ & $0,17-0,16-0,17-0,16$ \\
Tezduyar et al. (1992) & $1,38-1,40$ & $0,35-0,38$ & $0,169-0,170$ \\
Thomas, Nithiarasu e Bevan (2008) & 1,50 & 0,32 & - \\
Codina et al. (2006) & $1,49-1,52-1,53$ & $0,30-0,36-0,38$ & - \\
Gresho et al. (1984) & 1,76 & 1,48 & 0,180 \\
\hline
\end{tabular}

Finalmente, capturaram-se as disposições das linhas de corrente e de pressão no instante de tempo $\mathrm{t}=100,0$ para o integrador de segunda ordem com $\Delta t=0,05$, cuja distribuição é comparada com a apresentada por Najafi, Arefmanesh e Enjilela (2012) nas Fig. 4.20 e 4.21, onde novamente observa-se boa aproximação dos resultados.

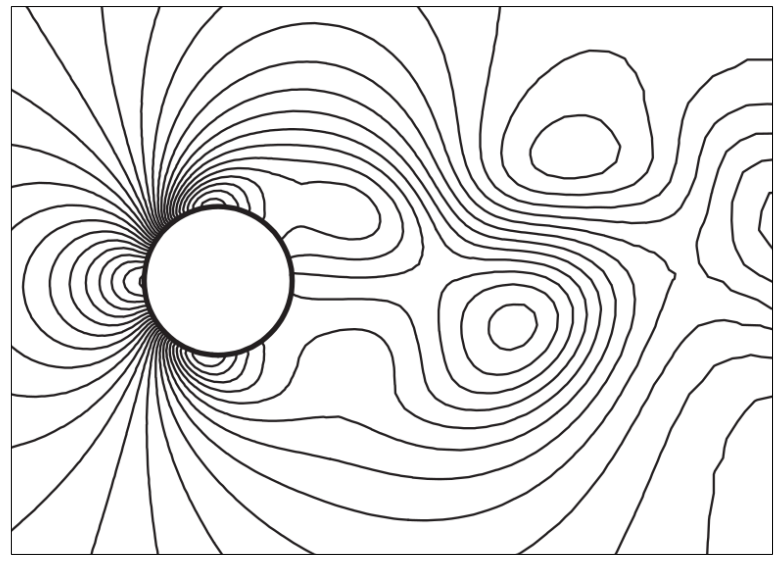

(a)

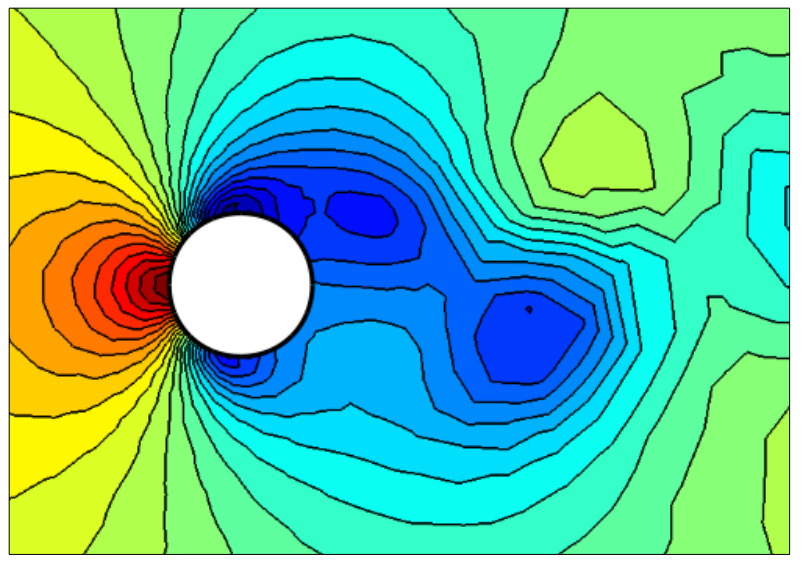

(b)

Figura 4.20: Linhas de pressão em t=100,0 (a) Najafi, Arefmanesh e Enjilela (2012) e (b) Presente trabalho. 


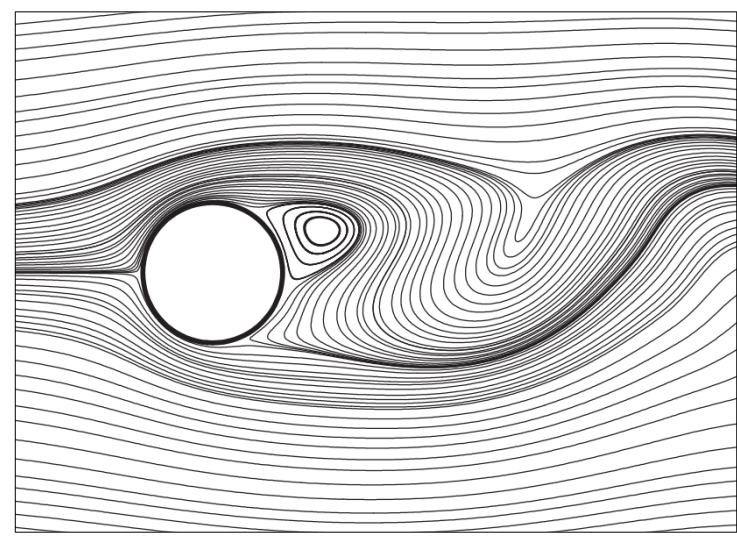

(a)

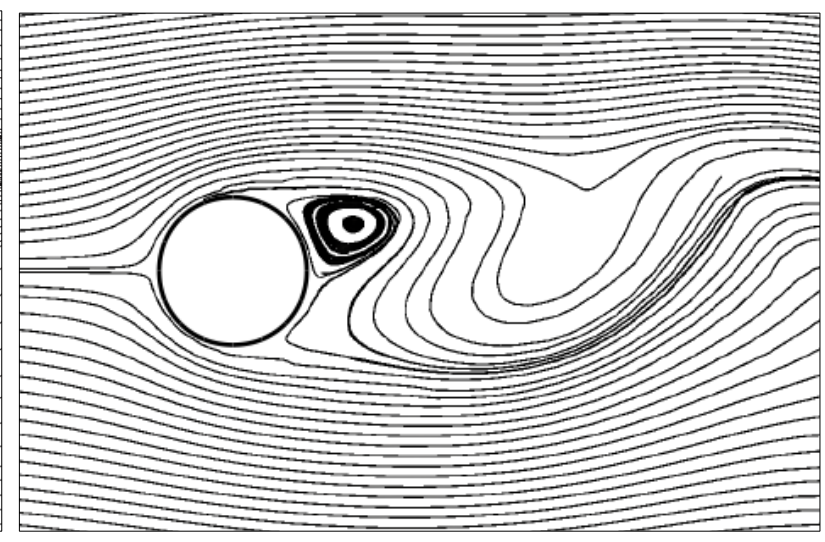

(b)

Figura 4.21: Linhas de corrente em $t=100,0$ (a) Najafi, Arefmanesh e Enjilela (2012) e (b) Presente trabalho. 
CAPÍtULO

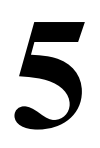

(2)

\section{ANÁLISE NUMÉRICA DA MECÂNICA DOS \\ SÓLIDOS}

Neste capítulo, a cinemática de movimentação de um sólido, introduzida no Capítulo 3 é aplicada a elementos finitos de casca. O problema de equilíbrio dinâmico é discretizado no tempo e o algoritmo desenvolvido por Coda e Paccola (2007) e Coda e Paccola (2008) com base no Método dos Elementos Finitos Posicional é aplicado em exemplos de verificação.

\subsection{A cinemática do MEF Posicional}

Na Mecânica dos Sólidos e, mais especificamente, na mecânica dos sólidos computacional, a deformação de um corpo é calculada a partir da mudança de uma configuração inicial $\left(\Omega_{0}\right)$ para uma final $(\Omega)$. Em termos práticos, existe uma função vetorial $\vec{f}$ que pode mapear essa alteração de estado. Quando se está trabalhando com o Método dos Elementos Finitos (MEF), uma das estratégias que se usa para a determinação dessa função é a utilização de um espaço adimensional, mapeado por coordenadas auxiliares $\xi_{1}, \xi_{2}$ (numa análise bidimensional), cujas equações governantes são integradas empregando-se a regra de quadratura adequada, como ilustra a Fig. 5.1 (CODA; PACCOLA, 2007).

A determinação das funções $\vec{f}^{0}$ e $\vec{f}^{1}$, por sua vez, se torna meramente numérica, de acordo com o MEF, já que as incógnitas do problema são interpoladas por funções aproximadoras (funções de forma) no domínio de um elemento finito.

Como já mencionado, o método dos elementos finitos posicional diferencia-se da abordagem clássica por substituir os parâmetros nodais de deslocamentos por posições, ou seja, os próprios valores das coordenadas nodais.

Tomando a discretização de um problema qualquer cujo elemento finito genérico é representado na Fig. 5.1, pode-se realizar o mapeamento das posições nas configurações inicial e deformada como: 


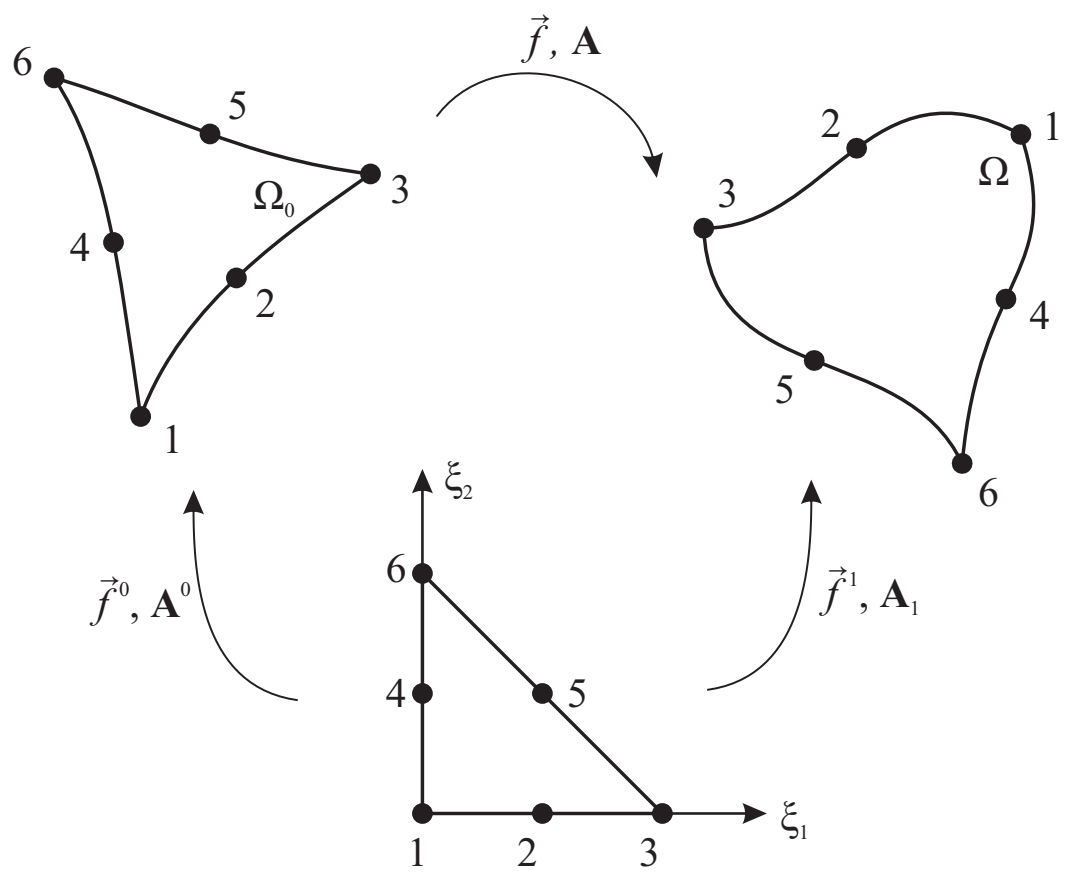

Figura 5.1: Cinemática do MEF Posicional.

Fonte: Adaptado de Coda e Paccola (2007).

$$
\begin{aligned}
f^{0} & =x_{i}\left(\xi_{1}, \xi_{2}, \xi_{3}, X_{l i}\right)=N_{l}\left(\xi_{1}, \xi_{2}, \xi_{3}\right) X_{l i}, \\
f^{1}=y_{i}\left(\xi_{1}, \xi_{2}, \xi_{3}, X_{l i}\right) & =N_{l}\left(\xi_{1}, \xi_{2}, \xi_{3}\right) Y_{l i},
\end{aligned}
$$

em que $x_{i}$ e $y_{i}$ são as $i$-ésimas posições de um ponto genérico pertencente a $\Omega_{0}$ e $\Omega$, respectivamente. $X_{l i}$ e $Y_{l i}$ são as coordenadas na direção $i$ do nó $l$ pertencente ao elemento finito em questão e $N_{l}$ é a função de forma associada ao nó $l$.

Para definir a função vetorial mudança de configuração $\vec{f}$, cujo domínio é $\Omega_{0}$ e imagem $\Omega$, pode-se realizar a aplicação linear de $\vec{f}^{1}$ sobre a imagem de $\left(\vec{f}^{0}\right)^{-1}$, isto é:

$$
\vec{f}=\vec{f}^{1} \circ\left(\vec{f}^{0}\right)^{-1} .
$$

Analogamente, tem-se o gradiente de $\vec{f}$ definido por:

$$
\mathbf{A}=\mathbf{A}^{1}\left(\mathbf{A}^{0}\right)^{-1},
$$

onde:

$$
\begin{aligned}
& \mathbf{A}^{0}=A_{i j}^{0}=f_{i, j}^{0}=\frac{\partial N_{l}\left(\xi_{1}, \xi_{2}, \xi_{3}\right)}{\partial x_{j}} X_{l i}, \\
& \mathbf{A}^{1}=A_{i j}^{1}=f_{i, j}^{0}=\frac{\partial N_{l}\left(\xi_{1}, \xi_{2}, \xi_{3}\right)}{\partial x_{j}} Y_{l i} .
\end{aligned}
$$

Desse modo, tem-se também o alongamento à direita de Cauchy-Green e a deformação de Green dados por: 


$$
\mathbf{C}=\mathbf{A}^{T} \mathbf{A}=\left[\left(\mathbf{A}^{1}\right)\left(\mathbf{A}^{0}\right)^{-1}\right]^{T}\left[\left(\mathbf{A}^{1}\right)\left(\mathbf{A}^{0}\right)^{-1}\right],
$$

$\mathrm{e}$

$$
\mathbf{E}=\frac{1}{2}(\mathbf{C}-\mathbf{I})=\frac{1}{2}\left(\mathbf{A}^{T} \mathbf{A}-\mathbf{I}\right)=\frac{1}{2}\left\{\left[\left(\mathbf{A}^{1}\right)\left(\mathbf{A}^{0}\right)^{-1}\right]^{T}\left[\left(\mathbf{A}^{1}\right)\left(\mathbf{A}^{0}\right)^{-1}\right]-\mathbf{I}\right\} .
$$

As Eq. 5.7 e 5.8 demonstram que conhecendo-se as posições iniciais e finais de cada nó da malha de elementos finitos pode-se determinar a deformação de Green do sólido também de forma nodal e, a partir desta, sua energia de deformação e estado de tensões.

\subsection{O MEF Posicional aplicado ao elemento finito de casca}

As cascas são elementos estruturais tridimensionais definidos por uma dimensão muito menor que as demais. Assim, pode-se desenvolver um modelo de movimentação descrito a partir de sua superfície média, reduzindo o número de graus de liberdade do problema, em comparação com elementos tridimensionais. Neste trabalho, a cinemática de Reissner para cascas é também adotada, de modo a levar em consideração as deformações geradas por cisalhamento.

$\mathrm{Na}$ abordagem realizada anteriormente para exemplificar o funcionamento do MEF Posicional, o elemento contava apenas com as posições nodais como graus de liberdade. $\mathrm{O}$ elemento de casca empregado neste trabalho, por sua vez, é isoparamétrico, podendo ser inicialmente curvo, triangular de 10 nós com aproximação por polinômios de Lagrange de ordem cúbica, cuja cinemática é apresentada na Fig. 5.2. Tal elemento conta com 7 graus de liberdade por nó: três correspondentes às coordenadas $x, y$ e $z$ do respectivo nó; três graus de liberdade definindo as componentes do vetor $\mathbf{g}$ inicialmente normal à superfície média e não necessariamente normal na configuração atual, de acordo com a cinemática de Reissner; e, por fim, a taxa de variação linear da deformação ao longo da espessura $a$, adicionada à formulação para se evitar o travamento pelo efeito de Poisson (CODA; PACCOLA, 2008).

O mapeamento das posições é realizado de forma análoga ao apresentado anteriormente, de acordo com as Eq. 5.5 e 5.6 em que $X_{l i}$ e $Y_{l i}$ correspondem às coordenadas dos nós, que agora descrevem a superfície média do elemento $x_{i}^{m}$ e $y_{i}^{m}$, respectivamente, nas configurações inicial e deformada, de acordo com a Fig. 5.2.

A taxa de variação linear da deformação $(a)$ é definida constante ao longo da espessura do elemento $\left(\xi_{3}\right)$, e interpolada na configuração atual como:

$$
a\left(\xi_{1}, \xi_{2}\right)=N_{j}\left(\xi_{1}, \xi_{2}\right) a_{j}
$$

onde $a_{j}$ é o valor da taxa no nó $j$.

Para completar o mapeamento do elemento de casca, assumem-se dois vetores, chamados vetores generalizados, $g^{0}$ e $g^{1}$ definidos como a diferença entre um ponto fora da superfície média e o seu correspondente pertencente à superfície média, como ilustrado na Fig. 5.2.

Sendo assim, a posição de um ponto qualquer no elemento pode ser definido pela adição 


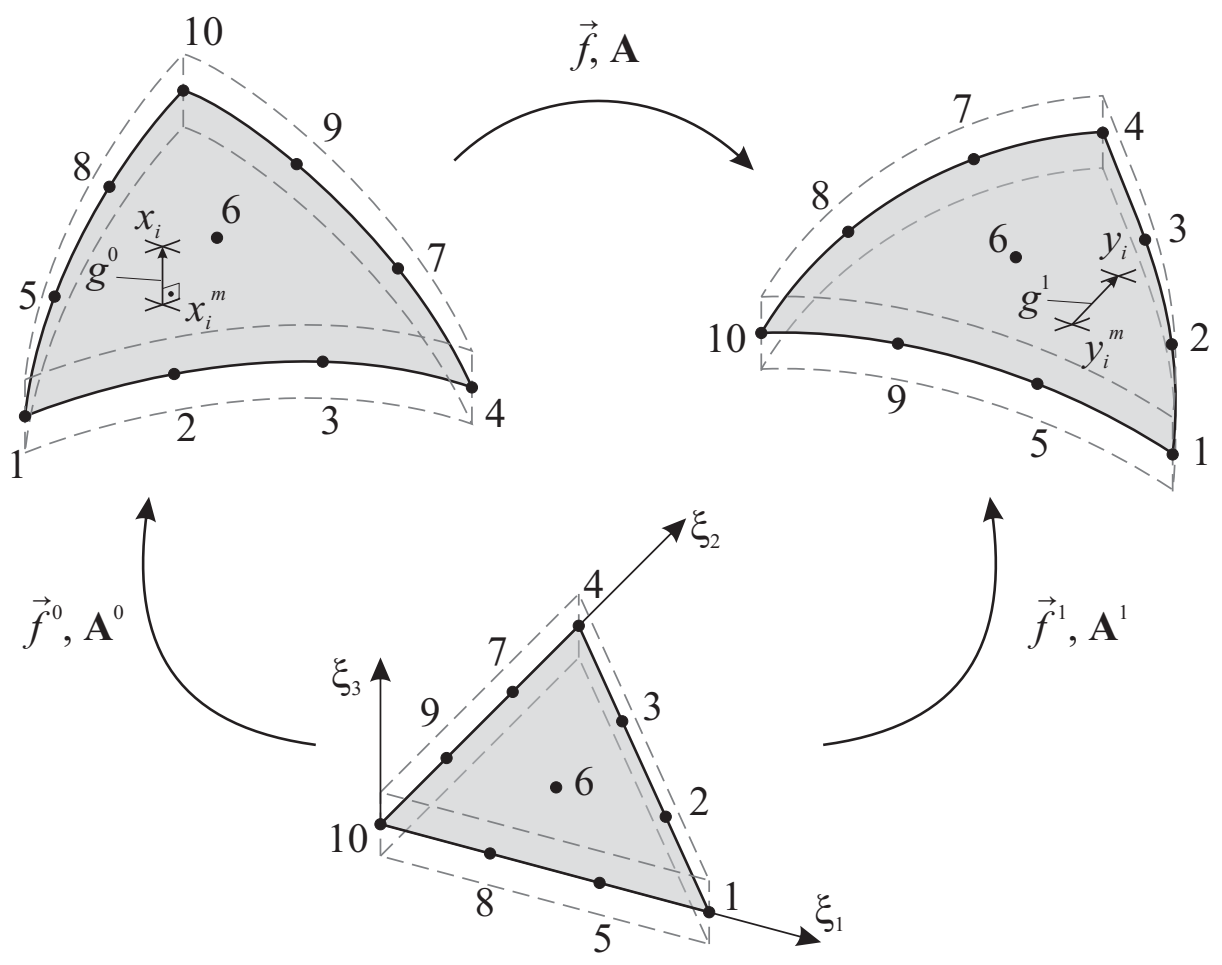

Figura 5.2: Cinemática do elemento finito de casca.

do vetor generalizado ao seu correspondente localizado na superfície média, considerando deformação constante na direção normal à superfície:

$$
\begin{aligned}
& x_{i}=x_{i}^{m}+g_{i}^{0}, \\
& y_{i}=y_{i}^{m}+g_{i}^{1} .
\end{aligned}
$$

Tendo em vista que as posições $x_{i}^{m}$ e $y_{i}^{m}$ já foram anteriormente parametrizadas (Eq. 5.5 e 5.6), reescreve-se os vetores $g_{i}^{0}$ e $g_{i}^{1}$ como:

$$
\begin{gathered}
g_{i}^{0}=\frac{h_{0}\left(\xi_{1}, \xi_{2}\right)}{2} \xi_{3} e_{i}^{0}\left(\xi_{1}, \xi_{2}\right), \\
g_{i}^{1}=\frac{h_{0}\left(\xi_{1}, \xi_{2}\right)}{2} N_{l} \bar{G}_{l i}\left[\xi_{3}+a \xi_{3}^{2}\right],
\end{gathered}
$$

em que $h_{0}$ representa a espessura da casca na configuração inicial, $e_{i}^{0}$ é o vetor unitário normal à superfície média inicial e $\bar{G}_{l i}$ são os valores do vetor generalizado (incógnita) no nó $i$ na configuração atual.

Como o vetor $e_{i}^{0}$ é aproximado em termos das funções de forma, pode-se trabalhar algebricamente nas Eq. 5.12 e 5.13 (ver Coda e Paccola (2007) e Sanches (2011)) de modo a compor as funções de mudança de configuração $f_{0}$ e $f_{1}$ pela soma de cada uma das parcelas referentes aos graus de liberdade definidos anteriormente para o elemento de casca:

$$
f_{i}^{0}=x_{i}=N_{j}\left(\xi_{1}, \xi_{2}\right) X_{j i}+\frac{h_{0}}{2} \xi_{3} N_{j}\left(\xi_{1}, \xi_{2}\right) e_{i j}^{0}
$$




$$
f_{i}^{1}=y_{i}=N_{j}\left(\xi_{1}, \xi_{2}\right) Y_{j i}+\frac{h_{0}}{2}\left[\xi_{3}+N_{j}\left(\xi_{1}, \xi_{2}\right) a_{j} \xi_{3}^{2}\right] N_{j}\left(\xi_{1}, \xi_{2}\right) \bar{G}_{i j}
$$

\subsection{Equilíbrio dinâmico do elemento de casca}

O equilíbrio dinâmico do elemento de casca em formulação posicional é realizado tomando-se como ponto de partida a Eq. 3.8, que define o equilíbrio dinâmico de um sólido genérico em forma fraca:

$$
\begin{aligned}
& \int_{\Omega_{0}}\left[\frac{1}{2} \rho_{0} \frac{\partial(\dot{\mathbf{y}} \cdot \dot{\mathbf{y}})}{\partial \mathbf{y}} \cdot \delta \mathbf{y}\right] d \Omega_{0}-\mathbf{F} \cdot \delta \mathbf{y} \\
- & \int_{\Omega_{0}}\left(\mathbf{b}_{0} \cdot \delta \mathbf{y}\right) d \Omega_{0}-\int_{\Gamma_{0}}(\mathbf{p} \cdot \delta \mathbf{y}) d \Gamma_{0}+\int_{\Omega_{0}}\left[\frac{\partial u_{e}}{\partial \mathbf{y}} \cdot \delta \mathbf{y}\right] d \Omega_{0}=0 .
\end{aligned}
$$

Trabalhando-se inicialmente com a derivada parcial presente na primeira parcela, que define as forças inerciais tem-se, agora em notação indicial:

$$
\frac{\partial\left(\dot{y}_{i} \dot{y}_{i}\right)}{\partial y_{j}}=\frac{\partial \dot{y}_{i}}{\partial y_{j}} \dot{y}_{i}+\dot{y}_{i} \frac{\partial \dot{y}_{i}}{\partial y_{j}}=2 \dot{y}_{i} \frac{\partial \dot{y}_{i}}{\partial y_{j}} \text {. }
$$

Tendo em vista que a aceleração e a velocidade de cada ponto é definida por:

$$
\ddot{y}_{i}=\frac{\partial \dot{y}_{i}}{\partial t} \text { e } \dot{y}_{j}=\frac{\partial y_{j}}{\partial t}
$$

e que a derivada com relação ao tempo é unidimensional, tem-se que:

$$
\frac{\partial \dot{y}_{i}}{\partial y_{j}}=\frac{\ddot{y}_{i}}{\dot{y}_{j}}
$$

Substituindo a Eq. 5.19 em 5.17 e atentando-se ao fato de que as posições são interpoladas como $y_{i}=N_{k} Y_{k i}$, a primeira parcela da Eq. 5.16 pode ser reescrita como:

$$
\int_{\Omega_{0}}\left[\frac{1}{2} \rho_{0} \frac{\partial(\dot{\mathbf{y}} \cdot \dot{\mathbf{y}})}{\partial \mathbf{y}} \cdot \delta \mathbf{y}\right] d \Omega_{0}=\int_{\Omega_{0}} \rho_{0} N_{i} N_{j} \ddot{\mathbf{Y}} \cdot \delta \mathbf{y} d \Omega_{0}
$$

em que $\ddot{\mathbf{Y}}$ contém os valores nodais de aceleração.

Em contrapartida, como demonstrado anteriormente, a energia específica de deformação para a lei constitutiva de Saint-Venant-Kirchhoff pode ser escrita como função da deformação de Green. Esta última, definida na Eq. 5.8, é dependente da posição do sólido. Assim, pode-se aplicar a regra da cadeia à ultima parcela da Eq. 5.16, do que resulta:

$$
\int_{\Omega_{0}}\left[\frac{\partial u_{e}}{\partial \mathbf{y}} \cdot \delta \mathbf{y}\right] d \Omega_{0}=\int_{\Omega_{0}}\left[\frac{\partial u_{e}^{S V K}}{\partial \mathbf{E}}: \frac{\mathbf{E}}{\partial \mathbf{Y}} \cdot \delta \mathbf{y}\right] d \Omega_{0}
$$

Assim, o equilíbrio definido pela Eq. 5.16 pode ser reescrito, agora em termos do MEF posicional da seguinte forma: 


$$
\begin{aligned}
& \int_{\Omega_{0}}\left[\rho_{0} N_{i} N_{j} \ddot{\mathbf{Y}} \cdot \delta \mathbf{y}\right] d \Omega_{0}-\mathbf{F} \cdot \delta \mathbf{y} \\
- & \int_{\Omega_{0}}\left(\mathbf{b}_{0} \cdot \delta \mathbf{y}\right) d \Omega_{0}-\int_{\Gamma_{0}}(\mathbf{p} \cdot \delta \mathbf{y}) d \Gamma_{0}+\int_{\Omega_{0}}\left[\frac{\partial u_{e}^{S V K}}{\partial \mathbf{E}}: \frac{\mathbf{E}}{\partial \mathbf{Y}} \cdot \delta \mathbf{y}\right] d \Omega_{0}=0 .
\end{aligned}
$$

Dada a arbitrariedade de $\delta \mathbf{y}$, tem-se por fim o equilíbrio dinâmico expresso por:

$$
\int_{\Omega_{0}}\left[\rho_{0} N_{i} N_{j} \ddot{\mathbf{Y}}\right] d \Omega_{0}-\mathbf{F}-\int_{\Omega_{0}} \mathbf{b}_{0} d \Omega_{0}-\int_{\Gamma_{0}} \mathbf{p} d \Gamma_{0}+\int_{\Omega_{0}}\left[\frac{\partial u_{e}^{S V K}}{\partial \mathbf{E}}: \frac{\mathbf{E}}{\partial \mathbf{Y}}\right] d \Omega_{0}=0,
$$

ou ainda, numa forma matricial tem-se:

$$
\mathbf{M} \ddot{\mathbf{Y}}+\mathbf{C} \dot{\mathbf{Y}}+\mathbf{F}^{\text {int }}-\mathbf{F}^{\text {ext }}=\mathbf{0}
$$

em que $\mathbf{M}$ é a matriz de massa (constante), $\mathbf{F}^{\text {ext }}$ representa o somatório das forças de campo, superfície e concentradas, $\mathbf{F}^{\text {int }}$ corresponde às forças elásticas e $\mathbf{C}$ é introduzida como a matriz de amortecimento, adotada proporcional à $\mathbf{M ~ e ~ K}$, segundo o modelo de Rayleigh.

\subsection{Integração temporal e processo de solução pelo método de Newton-Raphson}

Para realizar o avanço discreto no tempo, optou-se pela utilização do método Newmark- $\beta$ de integração temporal, já comprovadamente eficiente para o elemento de casca resolvido pelo MEF Posicional, como atestam os trabalhos de Coda e Paccola (2009), Sanches e Coda (2010a), Sanches e Coda (2010b), Sanches e Coda (2013), Sanches e Coda (2014). Deve-se notar que a formulação apresenta matriz de massa constante, ficando similar a um problema fisicamente não linear porém geometricamente linear. Considerando isso, Sanches e Coda (2013) e Coda e Paccola (2009) provam que para pequenas deformações, o método de Newmark conserva a quantidade de movimento e apresenta conservação de energia para a maioria dos problemas de engenharia (ver Sanches e Coda (2013) para maiores detalhes).

O método Newmark- $\beta$ caracteriza-se por ser implícito, sendo que as velocidades e posições nos instantes de tempo $t+\Delta t$ são determinadas a partir de:

$$
\begin{gathered}
\mathbf{Y}_{t+\Delta t}=\mathbf{Y}_{t}+\Delta t \dot{\mathbf{Y}}_{t}+\left(\frac{1}{2}-\beta\right) \Delta t^{2} \ddot{\mathbf{Y}}_{t}+\beta \Delta t^{2} \ddot{\mathbf{Y}}_{t+\Delta t} \\
\dot{\mathbf{Y}}_{t+\Delta t}=\dot{\mathbf{Y}}_{t}+(1-\gamma) \Delta t \ddot{\mathbf{Y}}_{t}+\gamma \Delta t \ddot{\mathbf{Y}}_{t+\Delta t}
\end{gathered}
$$

em que $\beta$ e $\gamma$ são parâmetros dependentes do comportamento assumido para a aceleração e $\Delta t$ é o valor do incremento de tempo. Admitindo aceleração constante no intervalo de um passo de tempo, adota-se $\gamma=1 / 2$ e $\beta=1 / 4$.

Aplicando-se as Eq. 5.25 e 5.26 à Eq. 5.24, tem-se para um instante de tempo $t+\Delta t$ a seguinte relação: 


$$
\left.\frac{\partial U_{e}}{\partial \mathbf{Y}}\right|_{t+\Delta t}-\mathbf{F}_{t+\Delta t}+\frac{\mathbf{M}}{\beta \Delta t^{2}} \mathbf{Y}_{t+\Delta t}-\mathbf{M} \mathbf{Q}_{t}+\mathbf{C} \mathbf{R}_{t}+\frac{\gamma \mathbf{C}}{\beta \Delta t} \mathbf{Y}_{t+\Delta t}-\gamma \Delta t \mathbf{C} \mathbf{Q}_{t}=\mathbf{0},
$$

com $\mathbf{Q}_{t}$ e $\mathbf{R}_{t}$ dependentes apenas de velocidades, acelerações e posições de passos anteriores e iguais a:

$$
\begin{gathered}
\mathbf{Q}_{t}=\frac{\mathbf{Y}_{t}}{\beta \Delta t^{2}}+\frac{\dot{\mathbf{Y}}_{t}}{\beta \Delta t}+\left(\frac{1}{2 \beta}-1\right) \ddot{\mathbf{Y}}_{t}, \\
\mathbf{R}_{t}=\dot{\mathbf{Y}}_{t}+\Delta t(1-\gamma) \ddot{\mathbf{Y}}_{t}
\end{gathered}
$$

Por se tratar de uma equação não linear, uma técnica numérica eficiente para a determinação da solução da Eq. 5.27 é o processo iterativo de Newton-Raphson. Seu emprego é realizado tomando-se a Eq. 5.27 e igualando-a a um vetor $\mathbf{s}$, inicialmente diferente de zero, chamado vetor de desbalanceamento mecânico:

$$
\left.\frac{\partial U_{e}}{\partial \mathbf{Y}}\right|_{t+\Delta t}-\mathbf{F}_{t+\Delta t}+\frac{\mathbf{M}}{\beta \Delta t^{2}} \mathbf{Y}_{t+\Delta t}-\mathbf{M} \mathbf{Q}_{t}+\mathbf{C} \mathbf{R}_{t}+\frac{\gamma \mathbf{C}}{\beta \Delta t} \mathbf{Y}_{t+\Delta t}-\gamma \Delta t \mathbf{C} \mathbf{Q}_{t}=\mathbf{s} .
$$

Expandindo $\mathbf{s}$ em série de Taylor e truncando nos termos de segunda ordem, tem-se:

$$
\mathbf{s}=\left.\mathbf{s}\right|_{\mathbf{Y}_{t}}+\left.\frac{\partial \mathbf{s}}{\partial \mathbf{Y}}\right|_{\mathbf{Y}_{t}} \Delta \mathbf{Y}+Q\left(\Delta \mathbf{Y}^{2}\right)
$$

Tendo em vista que a Eq. 5.27 é satisfeita quando $\mathbf{s}=\mathbf{0}$, o processo iterativo-incremental fica definido por:

$$
\left.\frac{\partial \mathbf{s}}{\partial \mathbf{Y}}\right|_{\mathbf{Y}_{t}} \Delta \mathbf{Y}=-\left.\mathbf{s}\right|_{\mathbf{Y}_{t}}
$$

ou ainda,

$$
\mathbf{H} \Delta \mathbf{Y}=-\left.\mathbf{s}\right|_{\mathbf{Y}_{t}} .
$$

em que $\mathbf{H}$ é a chamada matriz Hessiana, assim denominada por uma de suas parcelas ser referente à segunda derivada com relação à posição da energia específica de deformação. Como a lei constitutiva de Saint-Venant-Kirchhoff adotada é uma função convexa, a matriz Hessiana é positiva-definida.

Desse modo, $\mathbf{H}$ é determinada a partir da própria Eq. 5.30, tal que:

$$
\left.\frac{\partial \mathbf{s}}{\partial \mathbf{Y}}\right|_{t}=\left.\frac{\partial^{2} U_{e}}{\partial^{2} \mathbf{Y}}\right|_{t}+\frac{\mathbf{M}}{\beta \Delta t^{2}}+\frac{\gamma \mathbf{C}}{\beta \Delta t} .
$$

Sendo assim, o processo de solução, ilustrado no fluxograma da Fig. 5.3, consiste em determinar o valor de $\mathbf{Y}$ de tal modo que s seja menor que uma tolerância estabelecida, isto é, utilizar a configuração inicial como chute para o início do processo iterativo, seguido da resolução da Eq. 5.32 atualizando $\mathbf{Y}$ através de:

$$
\mathbf{Y}_{t+1}^{i+1}=\mathbf{Y}^{i}+\Delta \mathbf{Y}
$$


em que $i$ é o contador das iterações realizadas.

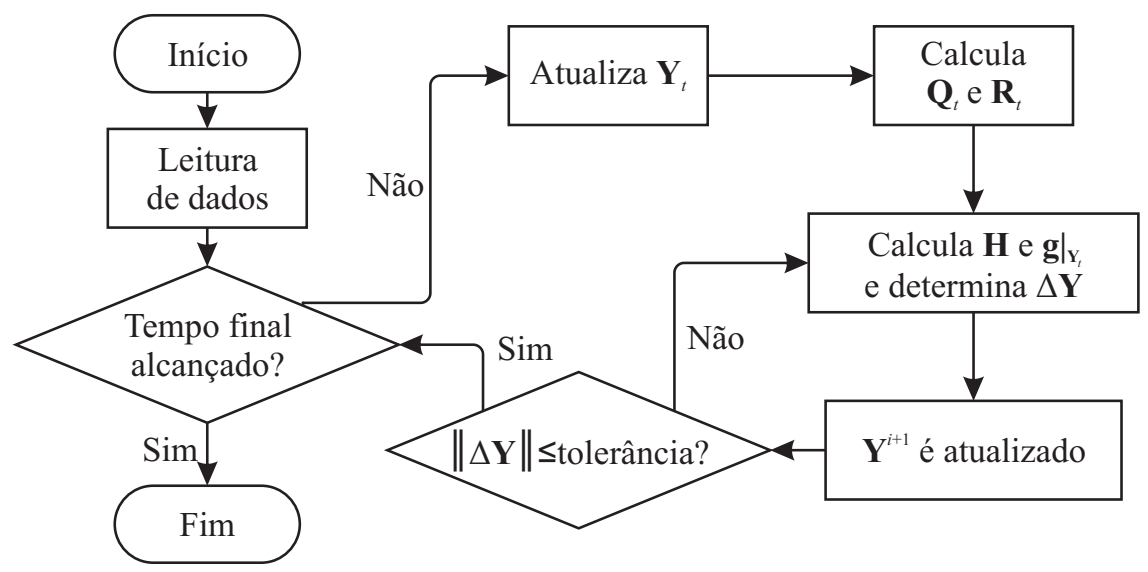

Figura 5.3: Fluxograma do procedimento de avanço temporal da solução.

\subsection{Exemplos de verificação do programa existente}

Nesta seção são apresentados exemplos numéricos com o objetivo de verificar o modelo numérico para análise não linear geométrica dinâmica de estruturas de casca proposto.

\subsubsection{Viga engastada com carregamento distribuído uniforme}

Este caso trata-se de uma viga engastada submetida a um carregamento distribuído uniforme, como apresentado na Fig. 5.4, e foi introduzido por Bathe, Ramm e Wilson (1975).
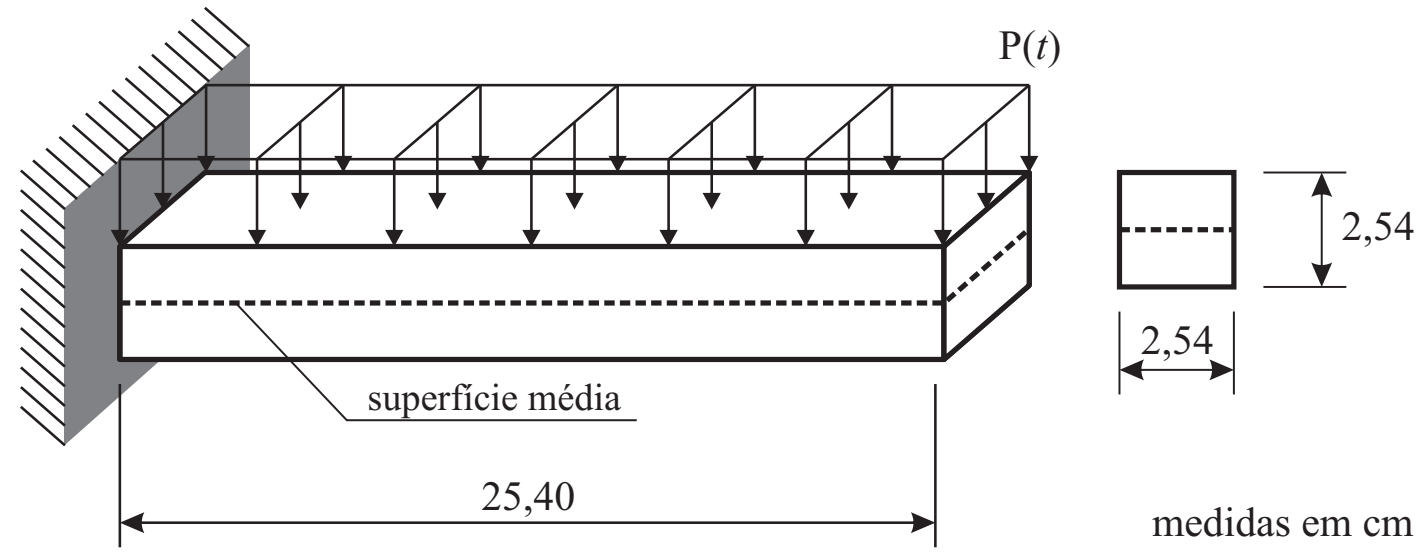

medidas em $\mathrm{cm}$

Figura 5.4: Geometria e condições de contorno da viga engastada com carregamento distribuído uniforme.

A viga possui seção transversal quadrada de lado igual a 2,54 cm e comprimento de 25,40 $\mathrm{cm}$, além de estar submedida a um carregamento vertical constante ao longo do tempo e igual 
a $-1,965 \mathrm{~N} / \mathrm{cm}^{2}$. A estrutura é composta também por um material com módulo de elasticidade $\mathbb{E}=82,74 \mathrm{MPa}$, coeficiente de Poisson $v=0,2$ e massa específica $\rho=1.0687 .10^{-5} \mathrm{~kg} / \mathrm{cm}^{3}$.

A modelagem por meio de elementos de casca é realizada discretizando-se a superfície média da viga, destacada na Fig. 5.4. Para tanto, empregou-se uma malha com 40 elementos finitos e 244 nós, num total de 1708 graus de liberdade, ilustrada na Fig. 5.5 com $e=2,54 \mathrm{~cm}$ e $l=25,40 \mathrm{~cm}$.

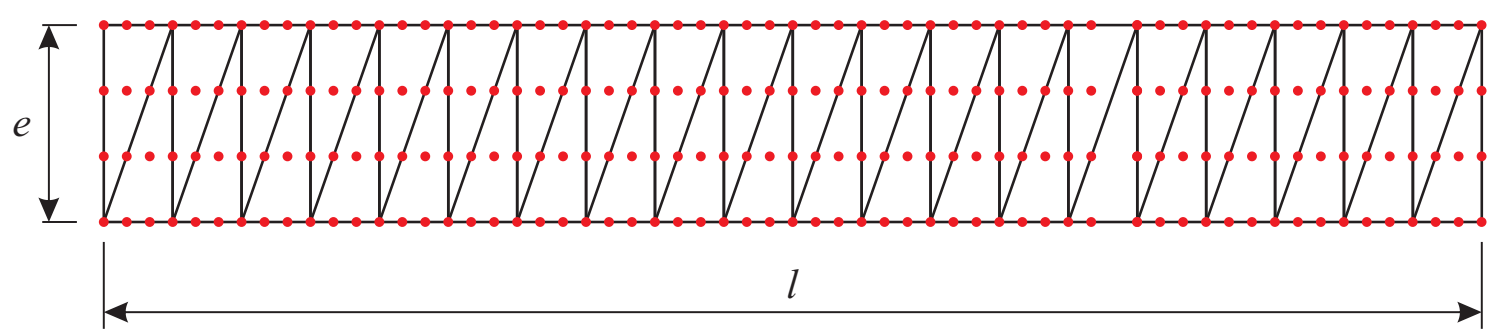

Figura 5.5: Malha de elementos finitos utilizada para a discretização da viga.

Cabe destacar que o carregamento distribuído é considerado do tipo não-conservativo, agindo sobre a estrutura como uma pressão, perpendicular ao eixo da viga. Este tipo de carregamento é introduzido na formulação modificando-se apenas a parcela correspondente às forças externas na equação de equilíbrio.

Diante disso, apresenta-se na Fig. 5.6 o deslocamento adimensionalizado na extremidade da viga em função do tempo obtida para um passo de tempo $\Delta t=1,35 \cdot 10^{-4} \mathrm{~s}$ em comparação com os resultados de Bathe, Ramm e Wilson (1975).

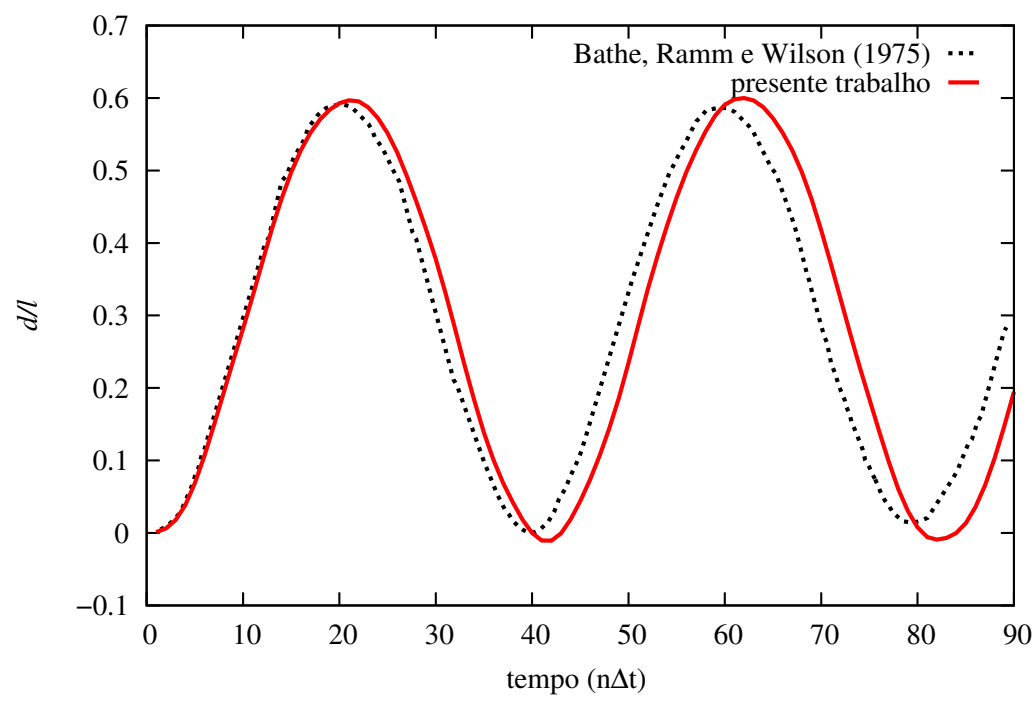

Figura 5.6: Deslocamento vertical da extremidade da viga em função do tempo.

As duas respostas se mostraram bastante semelhantes, notando-se uma diferença de fase e ainda que a resposta da referência diminui sua amplitude devido ao amortecimento numérico. 
Isso deve-se provavelmente aos diferentes elementos e formulações adotados. Além disso, Bathe, Ramm e Wilson (1975) simulam este caso utilizando apenas cinco elementos de chapa, ou seja, uma discretização pobre em comparação com a empregada neste trabalho.

Mesmo assim, percebe-se que a abordagem posicional se mostrou bastante eficiente para a reprodução deste problema claramente não linear geométrico, dada a amplitude dos deslocamentos desenvolvidos pela estrutura.

Por fim, na Fig. 5.7 apresentam-se as configurações deformadas da viga para diferentes instantes de tempo.

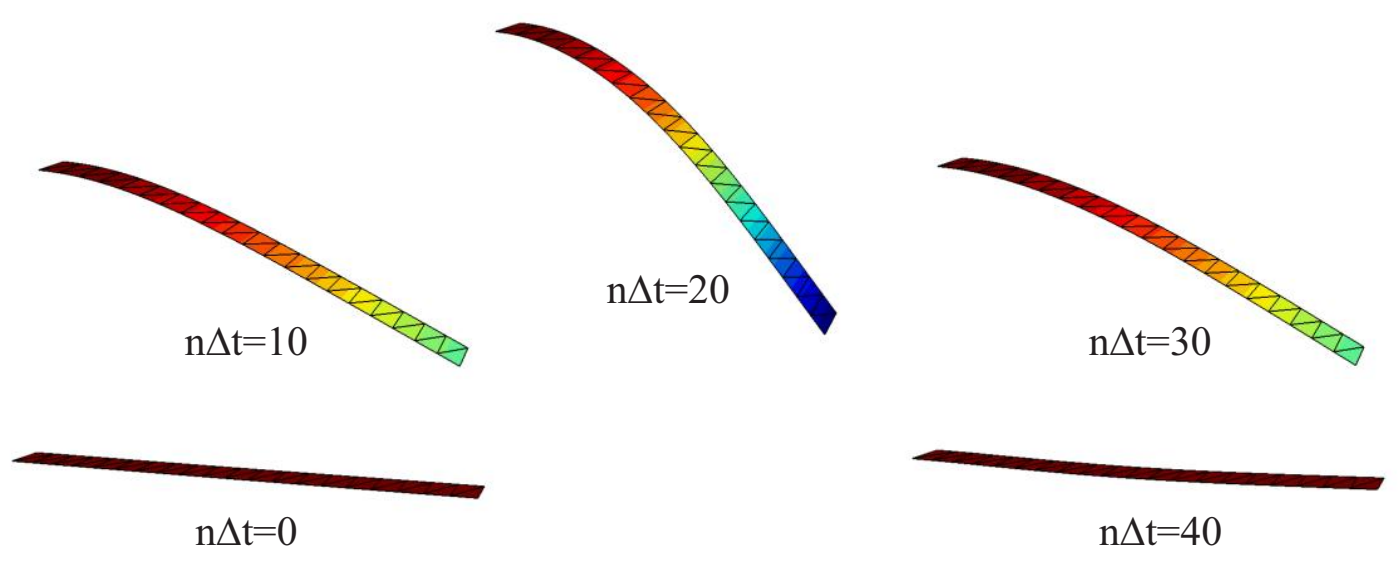

Deslocamento Y

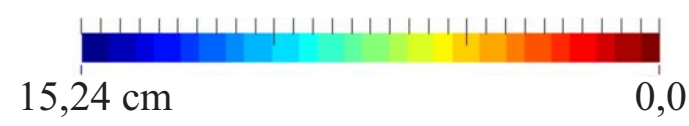

Figura 5.7: Configurações deformadas da viga engastada em diferentes instantes de tempo.

\subsubsection{Casca cilíndrica com Snap-through dinâmico}

Este exemplo foi proposto inicialmente por Kuhl e Ramm (1999), e vem sendo utilizado como benchmark para a verificação de formulações não lineares dinâmicas de cascas finas. Devido às não linearidades severas produzidas pelo efeito de snap-through, este caso é usado para testar se o algoritmo possui amortecimento numérico suficiente para suprimir os modos de alta frequência (ARGYRIS; PAPADRAKAKIS; MOUROUTIS, 2003).

O problema trata-se de uma casca cilíndrica submetida a uma carga concentrada central, cuja geometria é apresentada na Fig. 5.8. O carregamento varia com o tempo, sendo aumentado linearmente até atingir $0,2 \mathrm{~s}$ até um patamar de $50000 \mathrm{kN}$ e então mantido constante. Devido à simetria, optou-se pela simulação de apenas um quarto do problema, utilizando-se uma malha com 8 elementos finitos, 49 nós e 343 graus de liberdade.

Quanto às vinculações, as bordas curvas são consideradas completamente livres enquanto que as retas tem seu deslocamento restrito em todas as direções. Em relação ao material 


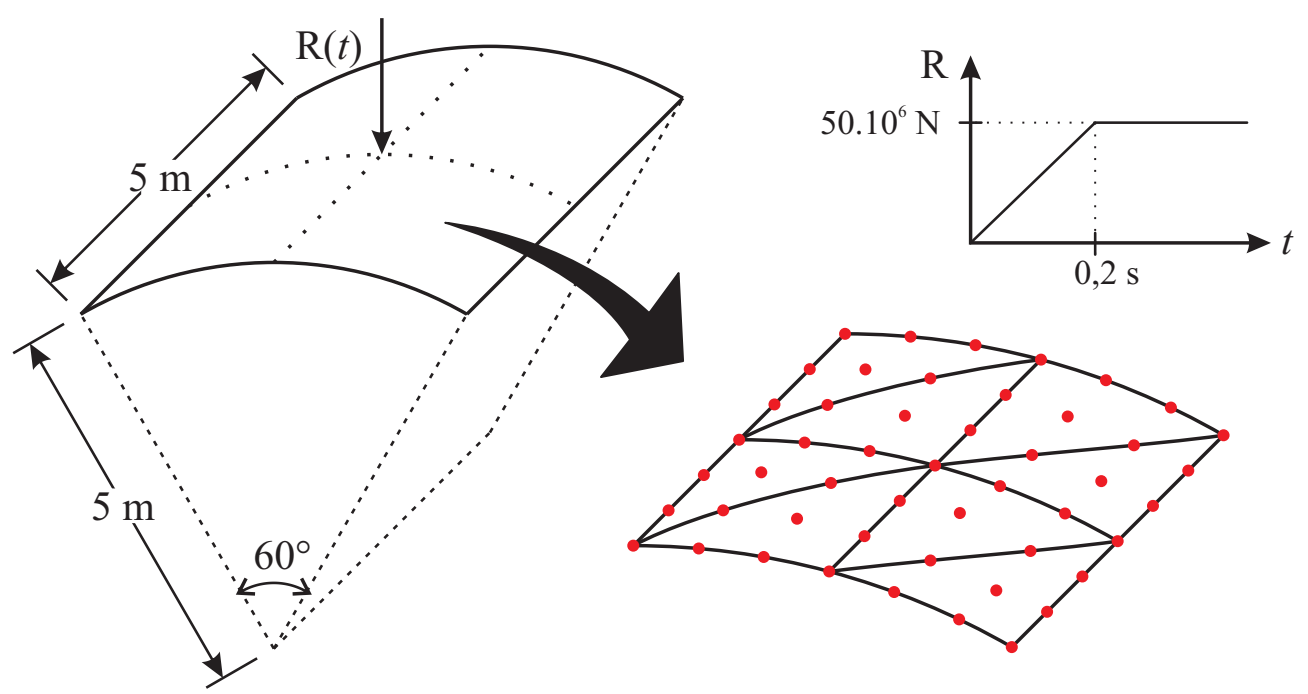

Figura 5.8: Geometria, discretização e comportamento temporal do carregamento para o exemplo de snap-through dinâmico.

constituinte, possui módulo de elasticidade $\mathbb{E}=200 \mathrm{GPa}, v=0,25$ e $\rho=10000 \mathrm{~kg} / \mathrm{m}^{3}$. Além disso, a casca possui espessura igual a $0,1 \mathrm{~m}$.

Os testes foram realizados para três diferentes passos de tempo ( $1,0 \mathrm{~ms}, 0,25 \mathrm{~ms}$ e 0,0625 $\mathrm{ms}$ ), cujos resultados para o deslocamento vertical no centro do vão são apresentados na Fig. 5.9, que podem ser comparados com os obtidos por Argyris, Papadrakakis e Mouroutis (2003), ilustrados na Fig. 5.10.

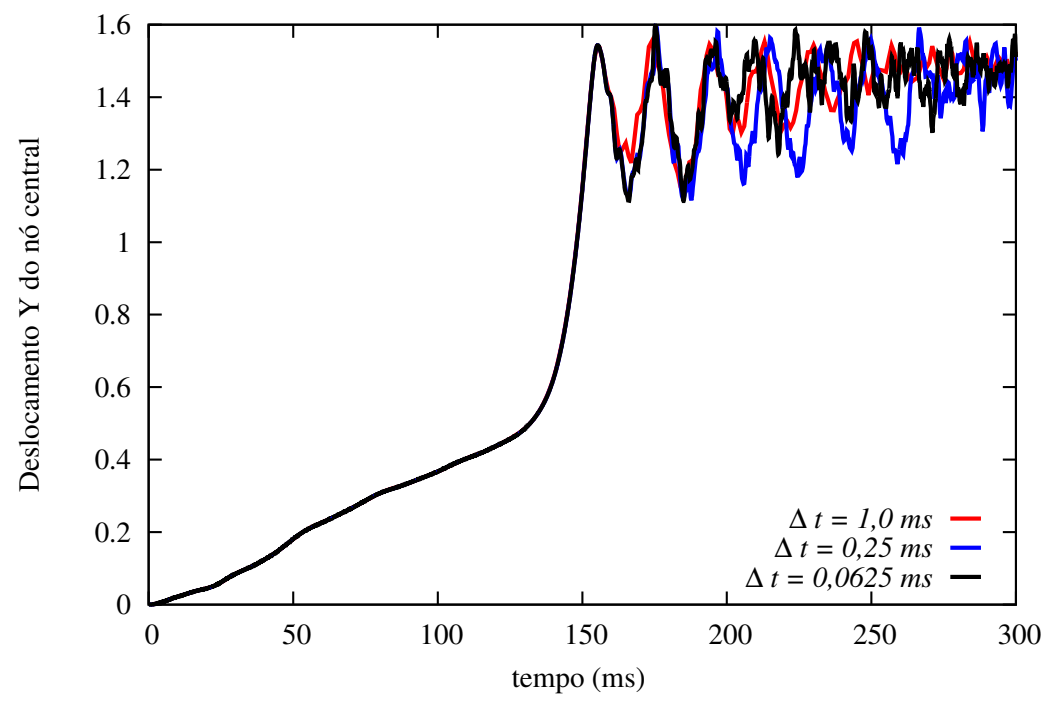

Figura 5.9: Deslocamento vertical no centro da casca em função do tempo.

Como pode-se perceber, os resultados estão de acordo com os de Argyris, Papadrakakis e Mouroutis (2003). Para todos casos analisados a estabilidade numérica foi preservada, melhorando-se a precisão quanto menor o passo de tempo empregado.

Por fim, apresentam-se na Fig. 5.11 as configurações deformadas do problema pré e pós 


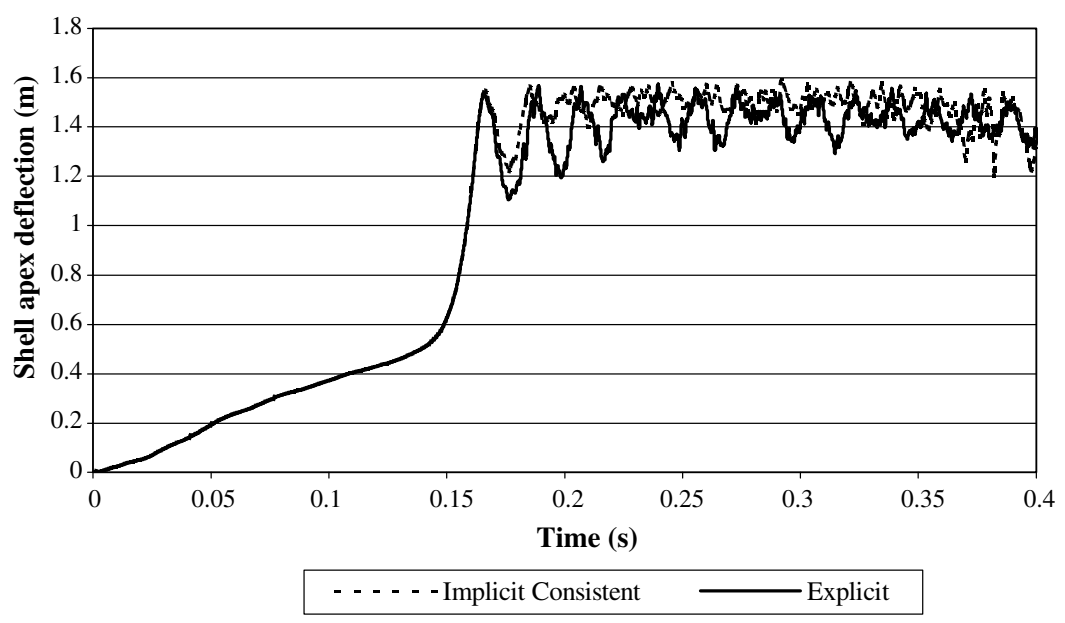

Figura 5.10: Resultados obtidos por Argyris, Papadrakakis e Mouroutis (2003) para o problema da casca cilíndrica com snap-through dinâmico.

snap-through.

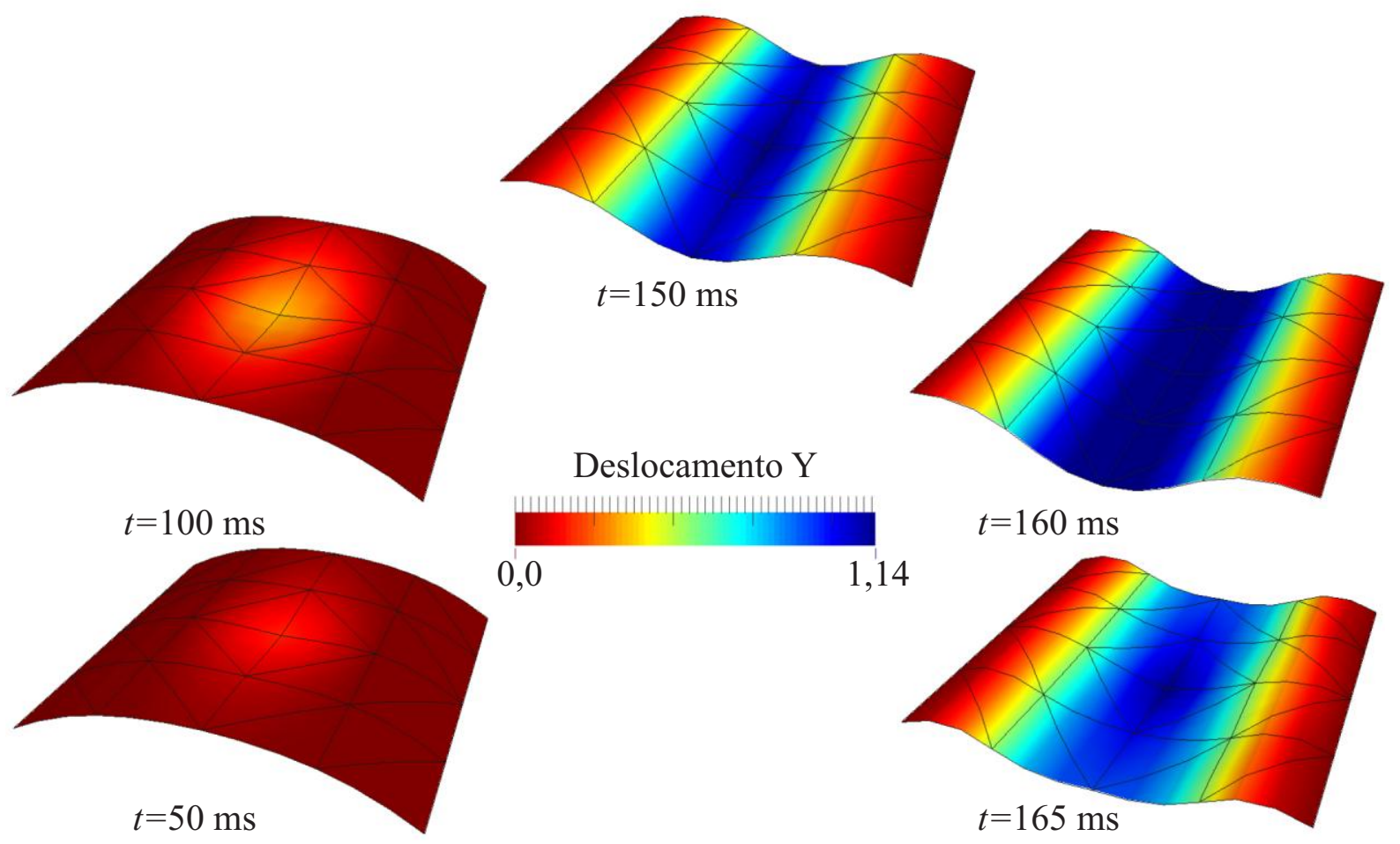

Figura 5.11: Configurações deformadas da casca para diferentes instantes de tempo. 
CAPÍtULO

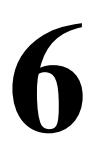

ACOPLAMENTO FLUIDO-CASCA

A partir dos desenvolvimentos realizados nos capítulos anteriores, tem-se a base matemática suficiente para definir o problema de interação fluido-estrutura incompressível, utilizando o esquema de acoplamento particionado fraco. As técnicas de transferência de forças e velocidades e de movimentação da malha do fluido são introduzidas para domínios com nós não necessariamente coincidentes. Em sequência, são apresentados exemplos de verificação da metodologia proposta.

\subsection{Condições de acoplamento - transferência de forças e ve- locidades}

Para exemplificar o problema de Interação Fluido-Estrutura, toma-se o domínio computacional $\Omega$ ilustrado na Fig. 6.1, composto pela união entre os domínios sólido $\Omega_{s}$ e fluido $\Omega_{f}$ sendo, por definição, a interface fluido-estrutura $\Gamma_{f s}$ a sua intersecção: $\Gamma_{f s}=\Omega_{s} \cap \Omega_{f}$.

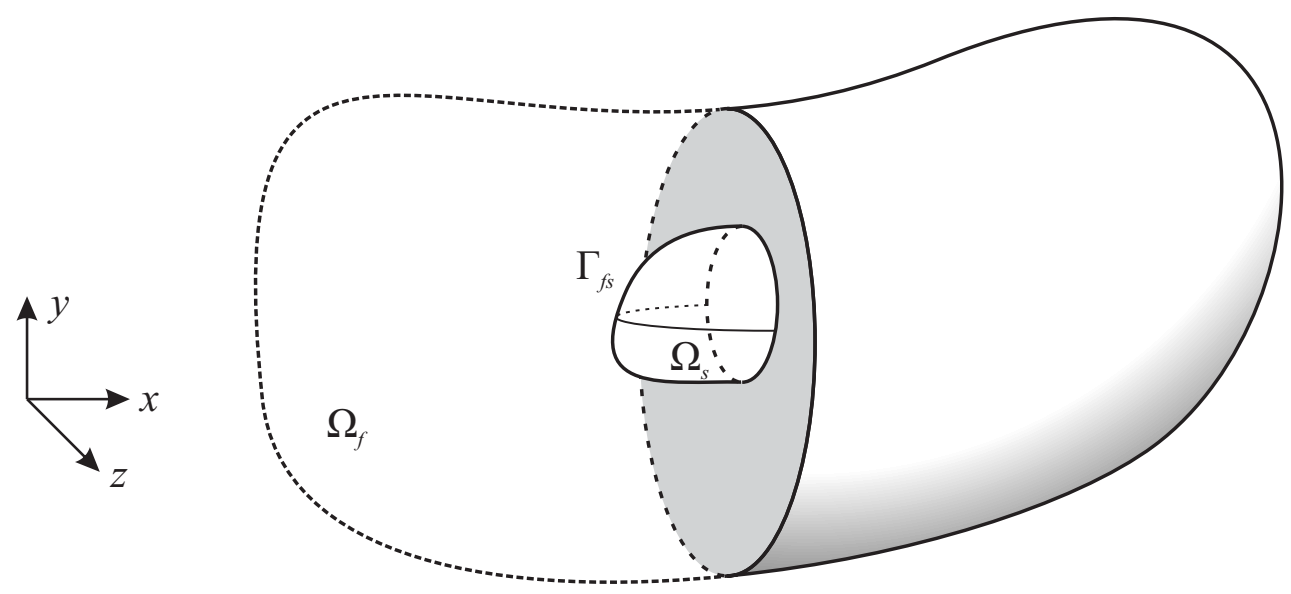

Figura 6.1: Representação esquemática do domínio computacional $\Omega$. 
Para que a interação fluido-estrutura seja corretamente modelada, são consideradas três condições referentes à interface sólido-fluido:

$i$. Os deslocamentos devem ser iguais, ou seja, a malha que descreve o domínio computacional do fluido deve adaptar-se à movimentação da estrutura;

ii. As velocidades de ambos os meios devem ser iguais;

iii. Deve haver o equilíbrio de forças nessa região.

Nesse sentido, duas abordagens são amplamente difundidas na literatura: monolítica e particionada. Na primeira, a interação e compatibilidade entre os meios é realizada em uma única formulação. Apesar de a abordagem monolítica geralmente ser considerada mais robusta, tem-se a desvantagem de que o método é computacionalmente mais caro em comparação com o modelo particionado, sendo considerado muitas vezes inviável para problemas de larga escala (HEIL; HAZEL; BOYLE, 2008).

O modelo de acoplamento particionado, adotado para este trabalho, apresenta por sua vez maior versatilidade, facilitando o tratamento matemático completamente distinto empregado para cada meio. A principal vantagem do método de acoplamento particionado é, para Badia, Nobile e Vergara (2008) e Teixeira e Awruch (2005), a modularidade, uma vez que contempla a utilização de métodos independentes para a resolução de cada um dos problemas físicos (sólido e fluido), permitindo que o avanço do código seja contínuo e com possíveis modificações em apenas um dos problemas físicos.

Ao empregar a descrição ALE para o fluido, a primeira condição de compatibilidade é alcançada por meio da deformação da malha do fluido. Essa tarefa apesar de aumentar o custo computacional, pode ser realizada também de forma independente dos processos de integração temporal e será abordada com mais detalhes no item 6.2.

As demais compatibilizações realizadas na interface entre os domínio são feitas de forma bidirecional: pela imposição das velocidades desenvolvidas pela estrutura nos nós da malha do fluido pertencentes a $\Gamma_{f_{s}}$ (condições de Dirichlet) e das forças advindas da pressão e viscosidade do fluido à estrutura (condições de Neumann). A esse esquema é dado o nome de DirichletNeumann, uma vez que o acoplamento ocorre por meio da imposição de condições de contorno desse tipo.

Algo que se deseja na implementação computacional é que os nós na interface $\Gamma_{f} s$ não sejam necessariamente coincidentes, como ilustra a Fig. 6.2.

Ao resolver cada problema, o que se obtém são as soluções nodais tanto do fluido quanto da estrutura, de modo que a imposição das condições de contorno também devem ser feita nó a nó. Desse modo, para cada nó $i_{f}$ pertencente ao domínio $\Omega_{f}$, deve-se encontrar a localização de um ponto $P s_{i}$ no domínio $\Omega_{s}$ mais próximo de $i_{f}$, tal que:

$$
\mathbf{x}\left(P s_{i}\right)=\mathbf{x}(i),
$$




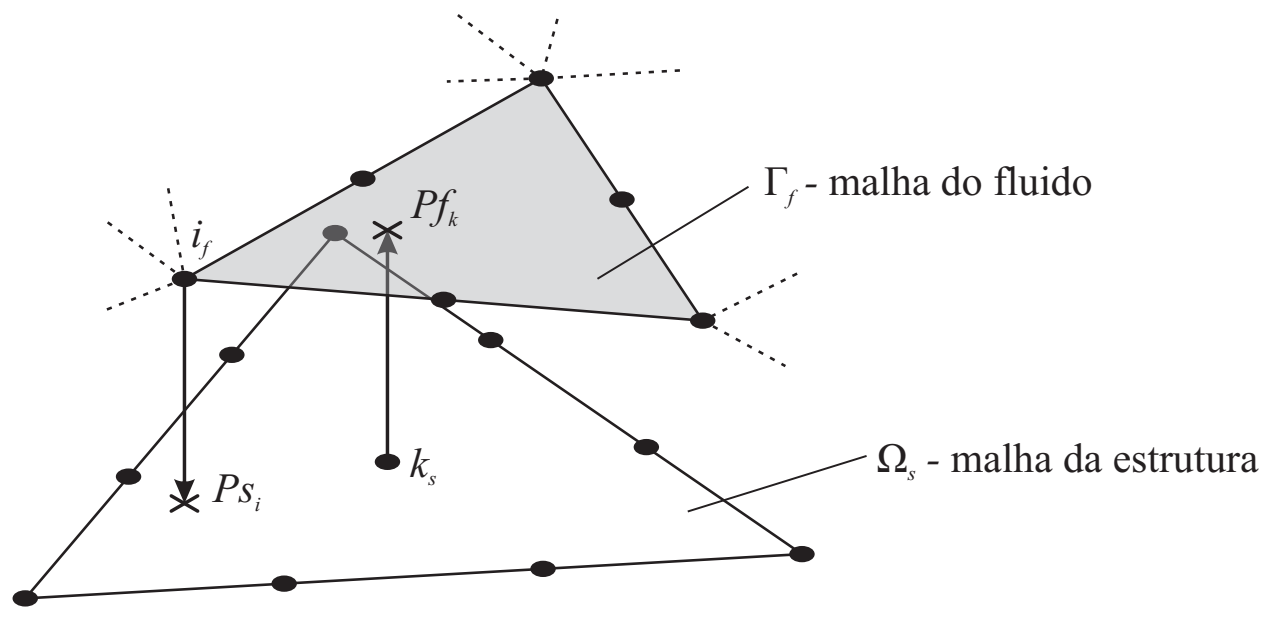

Figura 6.2: Nós pertencentes à interface $\Gamma_{f s}$.

Fonte: Adaptado de Sanches (2011).

em que $\mathbf{x}$ é o vetor de coordenadas cartesianas em relação ao sistema de referência.

Por outro lado, para um nó $k_{s}$, pertencente a $\Omega_{s}$, também deve-se buscar seu ponto correspondente $P f_{k}$ em $\Omega_{f}$, tal que:

$$
\mathbf{x}\left(P f_{k}\right)=\mathbf{x}(k) .
$$

Assim, na etapa de pré-processamento, os pontos $P f_{k}$ e $P s_{i}$ são identificados para cada nó da estrutura e nó do fluido pertencente à $\Gamma_{f s}$, definindo um vetor de coordenadas locais correspondente:

$$
\begin{gathered}
\boldsymbol{\xi}_{i}\left(P s_{i}\right)=\left(\xi_{1}, \xi_{2}, \xi_{3}\right), \\
\boldsymbol{\xi}_{k}\left(P f_{k}\right)=\left(\xi_{1}, \xi_{2}, \xi_{3}, \xi_{4}\right) .
\end{gathered}
$$

No caso de o escoamento ser viscoso e com condição de não-escorregamento em $\Gamma_{f s}$, a velocidade do nó $i(\mathbf{u}(i))$ é então prescrita por meio de condições de Dirichlet igual a $\dot{\mathbf{y}}\left(P s_{i}\right)$.

$$
\mathbf{u}\left(P s_{i}\right)=\sum_{j=1}^{10}\left(\mathbf{N}_{j} \dot{\mathbf{y}}_{j}\right),
$$

em que $\mathbf{N}$ é a função de forma associada aos elementos de casca e $\mathbf{\mathbf { y }}$ é o vetor de velocidades do sólido.

No caso de superfície lisa, apenas a componente normal é prescrita, conduzindo à seguinte relação:

$$
\mathbf{u}(i)=\mathbf{u}(i)+\left[\left(\dot{\mathbf{y}}\left(P s_{i}\right)-\mathbf{u}(i)\right) \cdot \mathbf{n}\right] \mathbf{n} .
$$

em que $\mathbf{n}$ é o vetor unitário normal à $\Gamma_{f s}$

Já para a condição de Neumann do nó $k$ da estrutura, as componentes de força no ponto $P f_{k}$ são calculadas e aplicadas à estrutura, de acordo com: 


$$
F_{j}(k)=\left[\tau_{j l} n_{l}-p n_{j}\right]_{P f_{k}}, \text { com } i \text { e } j=1,2 \text { e } 3 .
$$

\subsection{Movimentação dinâmica da malha do fluido}

Como mencionado anteriormente, a utilização da descrição ALE para o fluido adiciona uma etapa à resolução do problema de interação fluido-estrutura: o processo de movimentação dinâmica da malha do fluido.

$\mathrm{Na}$ simulação de problemas tridimensionais, esta tarefa acaba por demandar de um grande custo computacional. Diante disso, o que se propõe neste trabalho é o emprego de uma malha auxiliar composta por tetraedros de ordem cúbica com muito menos elementos do que a utilizada para a discretização do fluido, cujo domínio $\Omega_{a}$ é tal que $\Omega_{f} \subset \Omega_{a}$ e $\Gamma_{f}=\Gamma_{a}$, como ilustra a Fig. 6.3. Essa técnica justifica-se devido à movimentação da estrutura ser, em geral, muito mais suave do que o escoamento o que implica numa discretização menos refinada para captá-la.

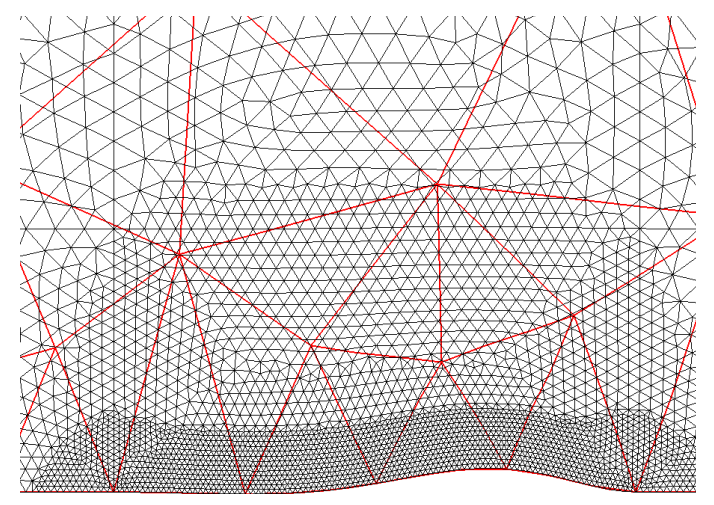

Figura 6.3: Representação da discretização do fluido imersa na malha auxiliar

Na fase de pré-processamento, para cada ponto $P$ pertencente à malha do fluido é realizada a busca do elemento $e l_{a}$ em que este encontra-se inserido, bem como suas coordenadas locais $\left(\xi_{1}^{a}(P), \xi_{2}^{a}(P), \xi_{3}^{a}(P)\right.$ e $\left.\xi_{4}^{a}(P)\right)$.

À medida que a malha auxiliar é atualizada, com base nos deslocamentos da estrutura, as posições $\left(x_{i}^{f}\right)$ e velocidades $\left(\omega_{i}\right)$ dos nós $P$ são então interpoladas de acordo com:

$$
\begin{aligned}
& x_{i}^{f}(P)=\sum_{j=1}^{20} N_{j}^{a}\left(\xi^{a}(P)\right) x_{i}^{a}(j), \\
& \omega_{i}(P)=\sum_{j=1}^{20} N_{j}^{a}\left(\xi^{a}(P)\right) \omega_{i}^{a}(j),
\end{aligned}
$$

em que $N_{j}^{a}$ representa a função de forma associada ao nó $j$ da malha auxiliar, $x_{i}^{a}$ representa as coordenadas dos nós que definem o elemento ao qual $x_{i}^{f}$ está contido e $\omega_{i}^{a}$ é a i-ésima componente de velocidade do nó $j$. 
Ainda é necessário deformar a malha auxiliar, de modo a acompanhar a movimentação da estrutura. Diante disso, diversas técnicas vem sendo desenvolvidas para realizar o procedimento de movimentação da malha de modo a garantir que a qualidade da discretização inicial seja preservada. Nesse sentido, optou-se pela metodologia proposta por Kanchi e Masud (2007) que realiza essa tarefa por meio de uma suavização Laplaciana (Laplacian smoothing).

Neste método procura-se determinar, para cada passo de tempo, o campo de deslocamentos da malha auxiliar (d) tal que:

$$
\begin{aligned}
& \nabla \cdot[(1+\eta) \nabla] \mathbf{d}=0, \text { em } \Omega_{a}, \\
& \mathbf{d}=\mathbf{y} \text { em } \Gamma_{f s} \mathrm{e} \\
& \mathbf{d}=\mathbf{0} \text { nos demais contornos de } \Omega_{a},
\end{aligned}
$$

em que $\mathbf{y}$ representa o deslocamento da estrutura.

Como pode-se perceber, a equação de Laplace (Eq. 6.10) é ponderada por um fator de restrição $\eta$. Para compreender sua função, considera-se uma malha qualquer que possua regiões mais refinadas do que as outras com o objetivo de capturar os efeitos em pequena escala tais como ondas de choque, desprendimento de vórtices, camada limite etc., normalmente próximas a $\Gamma^{f s}$. Nessas regiões, é necessário que a movimentação destes elementos seja feita com a menor distorção possível, mantendo o bom condicionamento da malha para os passos de tempo posteriores. Isso é alcançado com a utilização do fator $\eta$, definido pela equação 6.13 , que provê uma rigidez adicional aos elementos inversamente proporcional ao seu tamanho relativo na malha:

$$
\eta^{e}=\frac{1-V_{\min } / V_{\operatorname{máx}}}{V^{e} / V_{\operatorname{máx}}}
$$

em que $V_{\text {mín }}$ e $V_{\text {máx }}$ são, respectivamente, os volumes do menor e maior elementos da malha e $V^{e}$ é o volume do elemento em questão.

Para resolver o problema de valor de contorno definido pelas Eq. 6.10, 6.11 e 6.12, e tendo em vista que $\eta$ independe de $\mathbf{d}$, aplica-se o método dos resíduos ponderados à equação de Laplace:

$$
\int_{\Omega_{e}} \alpha\left(\nabla^{2} \mathbf{d}\right) \mathcal{F} d \Omega_{e}=0
$$

em que $\mathcal{F}$ é a função ponderadora e $\alpha=\left(1+\eta^{e}\right)$.

Integrando por partes a Eq. 6.14, tem-se:

$$
\int_{\Omega_{e}} \alpha \nabla \cdot(\mathcal{F} \nabla \mathbf{d}) d \Omega_{e}-\int_{\Omega_{e}} \alpha(\nabla \mathcal{F} \cdot \nabla \mathbf{d}) d \Omega_{e}=0
$$

Aplicando-se o teorema da divergência de Gauss à primeira parcela da Eq. 6.15, pode-se reescrevê-la como:

$$
\int_{\Gamma_{e}} \alpha(\mathcal{F} \nabla \mathbf{d}) \cdot \mathbf{n} d \Gamma_{e}-\int_{\Omega_{e}} \alpha(\nabla \mathcal{F} \cdot \nabla \mathbf{d}) d \Omega_{e}=0
$$


em que $\mathbf{n}$ representa o vetor normal a $\Gamma_{e}$.

Considerando-se a aproximação clássica de elementos finitos, tem-se que d é interpolado em $\Omega_{e}$ segundo:

$$
\mathbf{d}\left(\mathbf{x}^{a}\right)=\sum_{k=1}^{K}\left[\mathbf{N}_{k}^{a}\right]^{T}\left(\mathbf{x}^{a}\right) \overline{\mathbf{d}}^{k}
$$

em que $K$ é o número total de nós da malha auxiliar, $\overline{\mathbf{d}}$ é o deslocamento nodal e, como definido anteriormente, $\mathbf{N}^{a}$ são as funções interpoladoras, tais que:

$$
\left[\mathbf{N}^{a}\right]^{T}=\left[\begin{array}{llll}
N_{1}^{a} \mathbf{I} & N_{2}^{a} \mathbf{I} & \cdots & N_{K}^{a} \mathbf{I}
\end{array}\right],
$$

e I é o tensor identidade de dimensão três.

Aplicando-se a Eq. 6.17 e o método de $\operatorname{Galerkin}\left(\mathcal{F}=\mathbf{N}^{a}\right)$ à Eq. 6.16, tem-se o problema de valor de contorno (PVC) definido por:

$$
\left[\int_{\Gamma_{e}} \alpha\left(\mathbf{N}^{a} \nabla \mathbf{N}^{a}\right) \cdot \mathbf{n} d \Gamma_{e}\right] \overline{\mathbf{d}}-\left[\int_{\Omega_{e}} \alpha\left(\nabla \mathbf{N}^{a} \cdot \nabla \mathbf{N}^{a}\right) d \Omega_{e}\right] \overline{\mathbf{d}}=0 .
$$

Levando em consideração que o caso em questão trata-se de um problema de Dirichlet, com valores prescritos em todos os contornos (Eq. 6.11 e 6.12), a primeira parcela da Eq. 6.19 é nula, podendo-se reescrever:

$$
\left[\int_{\Omega_{e}} \alpha\left(\nabla \mathbf{N}^{a} \cdot \nabla \mathbf{N}^{a}\right) d \Omega_{e}\right] \overline{\mathbf{d}}=0 .
$$

Por fim, o problema de movimentação da malha pode ser reescrito de forma discreta com abordagem pelo MEF e em notação matricial como:

$$
\begin{gathered}
\mathbf{B} \overline{\mathbf{d}}=\mathbf{0}, \\
\overline{\mathbf{d}}=\mathbf{y} \text { em } \Gamma_{f s} \mathrm{e} \\
\overline{\mathbf{d}}=\mathbf{0} \text { nos demais contornos de } \Omega_{a},
\end{gathered}
$$

com as componentes de $\mathbf{B}$ dadas por:

$$
B_{i j}=\int_{\Omega_{e}} \alpha\left(\frac{\partial N_{i}^{a}}{\partial x} \frac{\partial N_{j}^{a}}{\partial x}+\frac{\partial N_{i}^{a}}{\partial y} \frac{\partial N_{j}^{a}}{\partial y}+\frac{\partial N_{i}^{a}}{\partial z} \frac{\partial N_{j}^{a}}{\partial z}\right) d \Omega_{e}
$$

\subsection{Processo de acoplamento particionado fraco}

A principal vantagem do método de acoplamento particionado consiste na possibilidade de resolução dos problemas físicos separadamente. Em alguns casos, é necessário que se utilizem passos de tempo diferentes para o avanço temporal em cada um dos meios. No entanto, os exemplos de interesse neste trabalho podem ser simulados com passos de tempo iguais para o fluido e a estrutura.

O avanço da solução com o tempo através do método de acoplamento particionado fraco 
do tipo Dirichlet-Neumann é realizado de acordo com o esquema apresentado na Fig. 6.4.

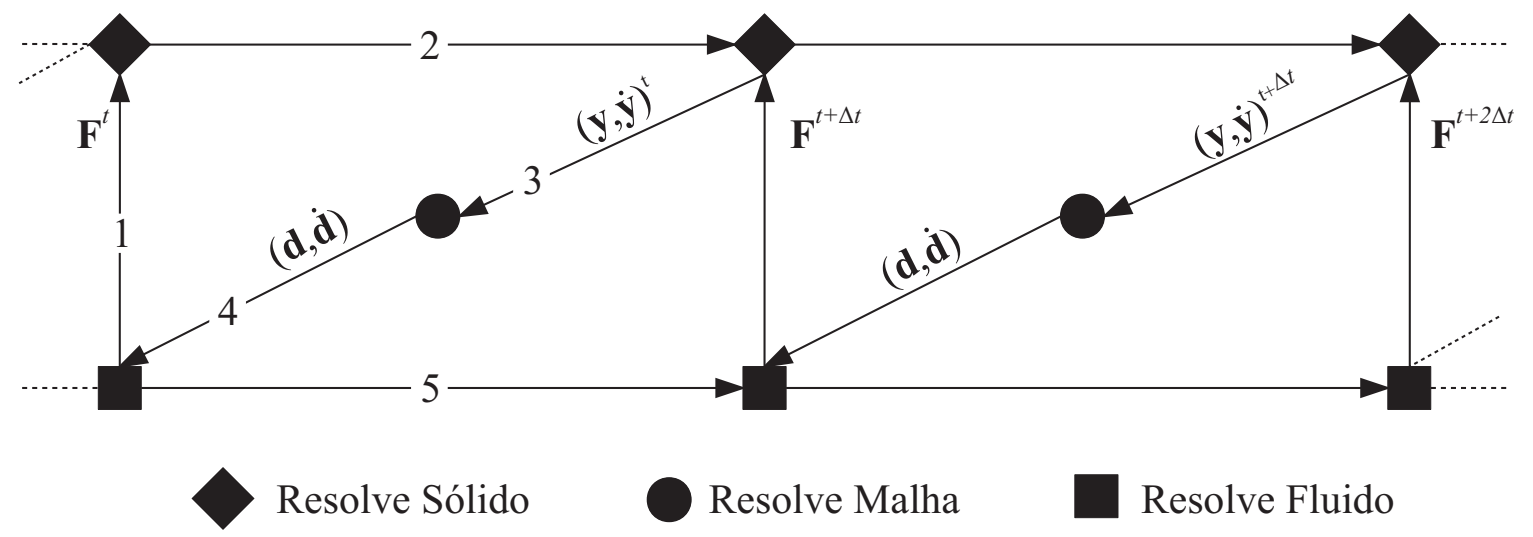

Figura 6.4: Esquema de acoplamento particionado fraco.

Desse modo, para um determinado passo de tempo $t$, a integração temporal ocorre de acordo com os seguintes passos:

1. A partir das tensões viscosas e pressões provenientes da análise prévia do fluido, são calculadas as componentes de força $\left(\mathbf{F}^{t}\right)$ e, em seguida aplicadas à estrutura;

2. A estrutura avança no tempo, determinando-se sua nova configuração deslocada $\left(\mathbf{y}^{t}\right)$ e a velocidade no movimento $\left(\dot{\mathbf{y}}^{t}\right)$;

3. Uma nova configuração para a malha do fluido é calculada com base nas novas posições $\left(\mathbf{y}^{t}\right)$ da estrutura;

4. Com a malha atualizada e com base na velocidade da estrutura $\left(\dot{\mathbf{y}}^{t}\right)$, calculam-se $\mathbf{u}^{t+\Delta t} \mathrm{e}$ $p^{t+\Delta t}$

5. O fluido avança no tempo e retorna ao passo 1 até que se atinja o tempo final da análise.

\subsection{Verificação do problema acoplado}

Nesta seção, três exemplos de aplicação são apresentados com o intuito de verificar o esquema de acoplamento implementado.

\subsubsection{Cavidade quadrada com fundo flexível}

Este exemplo trata-se da expansão do problema da cavidade quadrada, apresentado na seção 4.4.1, agora considerando-a com fundo flexível e velocidade tangencial oscilatória. As condições de contorno, bem como os parâmetros adimensionalizados são apresentados na Fig. 6.5. Observa-se que em $z=0$ e $z=e$ são aplicadas velocidade normal nula para o fluido e engastamento móvel para a estrutura.

O perfil de velocidades oscilatório faz com que o número de Reynolds do escoamento varie entre 0 a 200. De acordo com os resultados obtidos para a cavidade rígida, no item 4.4.1, 


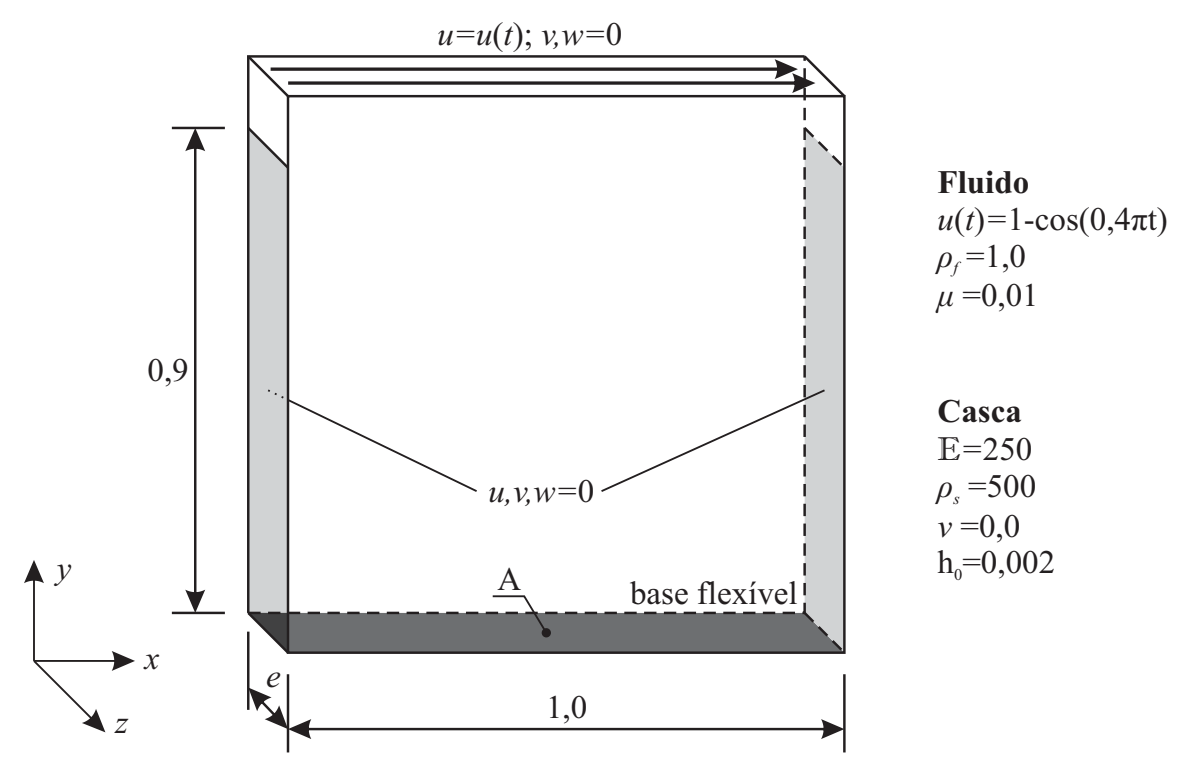

Figura 6.5: Geometria e condições de contorno do problema da cavidade com fundo flexível.

para esta ordem de grandeza, as malhas 1, 2 e 3 empregadas previamente apresentaram bons resultados e são novamente aplicadas a este exemplo para a discretização do fluido.

Para a casca, utiliza-se também a malha do exemplo 5.5.1, com 40 elementos e 244 nós, $l=1$ e $e=0,1$. Quanto à estrutura, encontra-se simplesmente apoiada nas extremidades.

No que concerne à movimentação da malha do fluido, utilizou-se a malha auxiliar apresentada na Fig. 6.6. Tal discretização conta com 1468 nós e 222 elementos, caracterizando o problema de movimentação da malha com um total de 4404 graus de liberdade. Em termos comparativos, o mesmo procedimento empregando-se diretamente as malhas 1, 2 e 3 produziria problemas de 11211, 31338 e 55311 graus de liberdade, respectivamente.

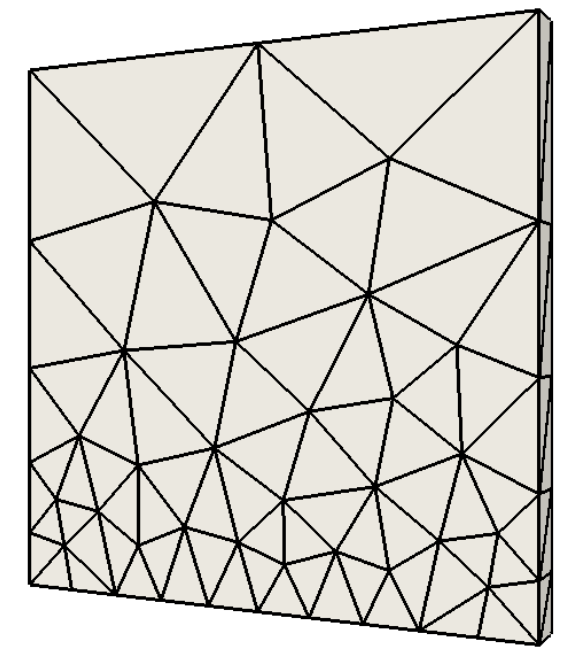

Figura 6.6: Malha auxiliar para movimentação do domínio do fluido.

Em relação ao avanço temporal, utilizou-se um passo de tempo de $\Delta t=0,1$. Um ponto relevante sobre esse aspecto é o fato de que assim como reportado por Förster, Wall e Ramm 
(2007), não obtiveram-se respostas estáveis ao utilizar o integrador de segunda ordem, mesmo para passos de tempo menores. Desse modo, apenas resultados para o integrador de primeira ordem são apresentados.

Os resultados obtidos estão de acordo com os de Mok (2001) e Gerbeau e Vidrascu (2003), de modo que a Fig. 6.7 apresentada as curvas de deslocamento vertical em função do tempo do ponto A, localizado no centro da casca, para as três malhas.

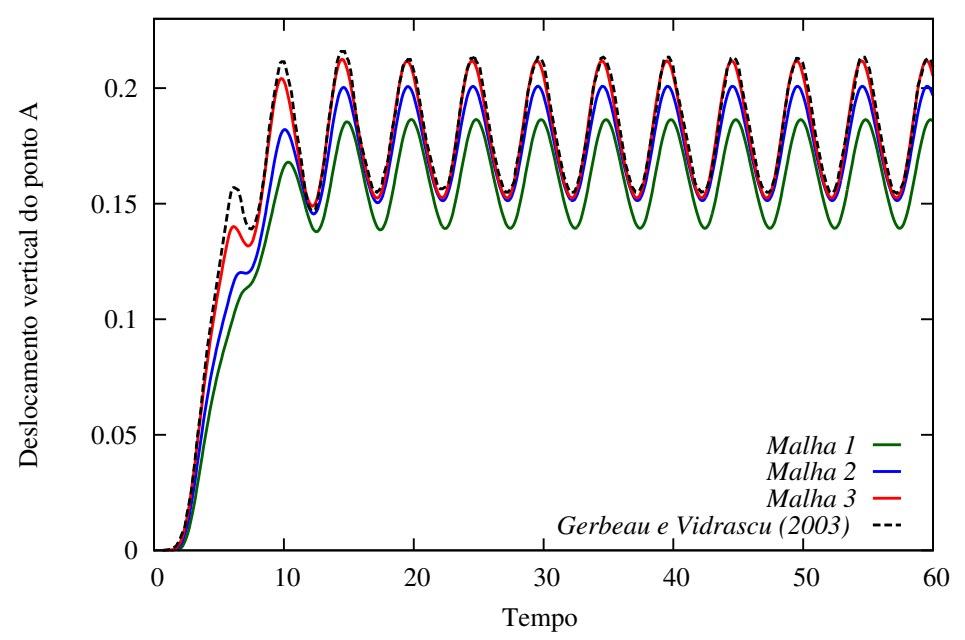

Figura 6.7: Deslocamento vertical do ponto A em função do tempo.

Como esperado, à medida que a qualidade da discretização aumenta, tem-se um resultado mais próximo da referência, que no caso de Mok (2001), por exemplo, empregou uma malha de $32 \times 32$ elementos quadrilaterais do tipo Q1-Q1.

Por fim, as Fig. 6.8 e 6.9 apresentam as configurações do campo de pressão e das linhas de corrente para diferentes instantes de tempo, bem como as configurações deformadas da malha 3 e da malha auxiliar. É possível observar também o regime de grandes deslocamentos ao qual a estrutura é submetida, além do complexo funcionamento do fenômeno acoplado.

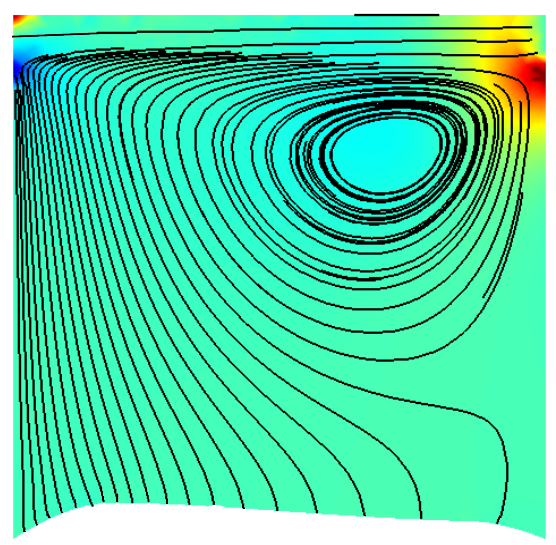

(a) $t=3,5$

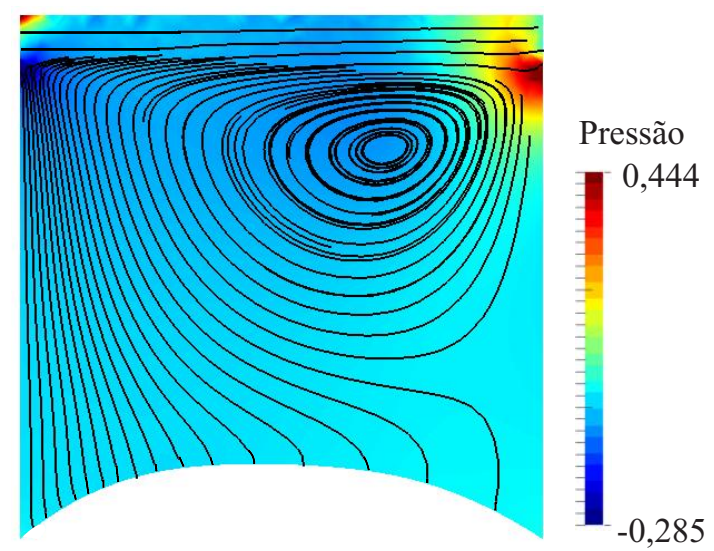

(b) $t=8,0$

Figura 6.8: Distribuição de pressões e linhas de corrente para diversos instantes de tempo. 


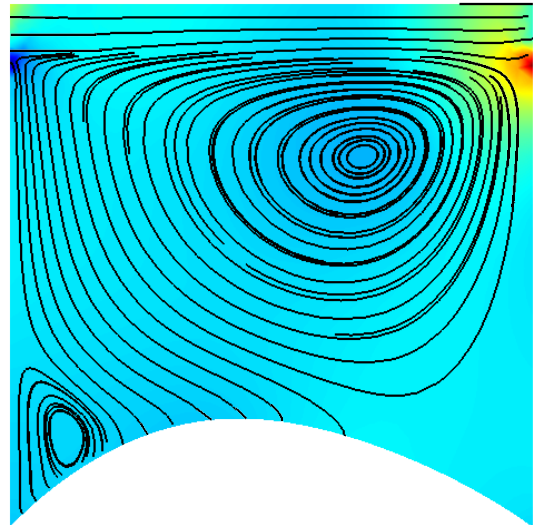

(c) $t=14,0$

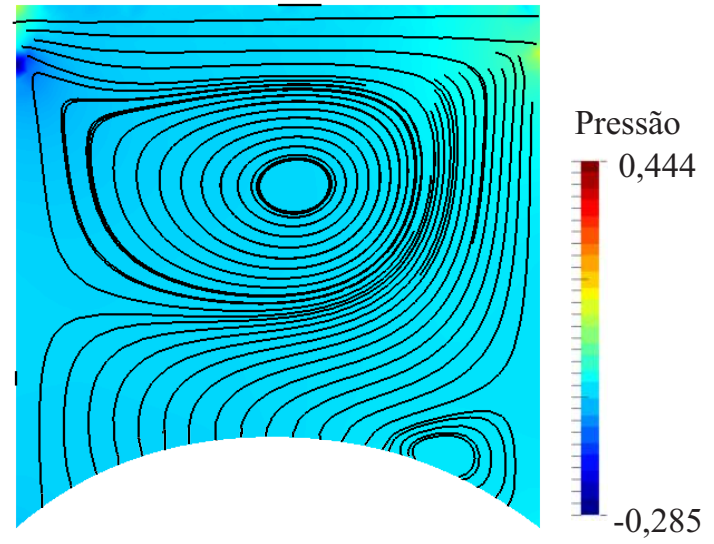

(d) $t=21,0$

Figura 6.8: Distribuição de pressões e linhas de corrente para diversos instantes de tempo continuação.

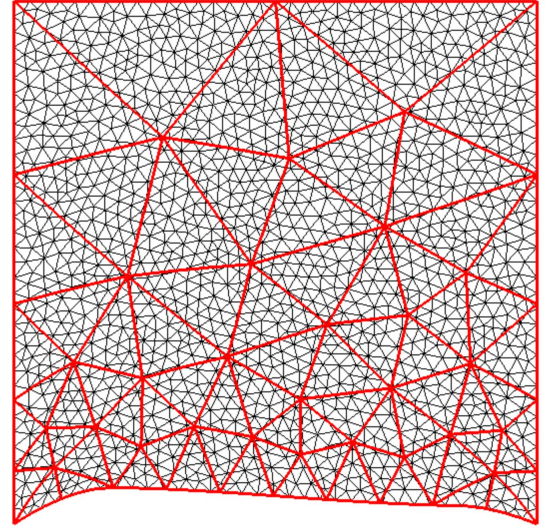

(a) $t=3,5$

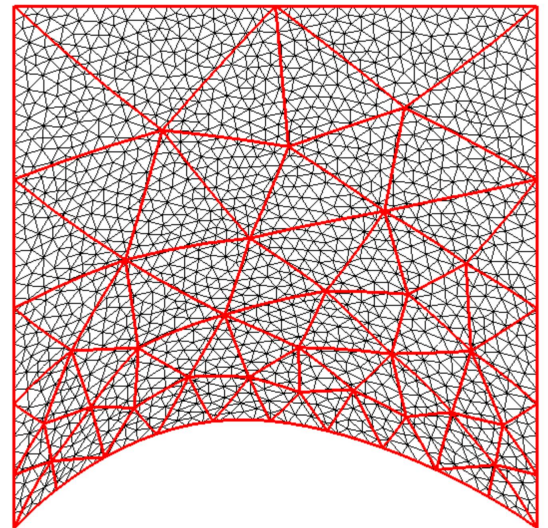

(c) $t=14,0$

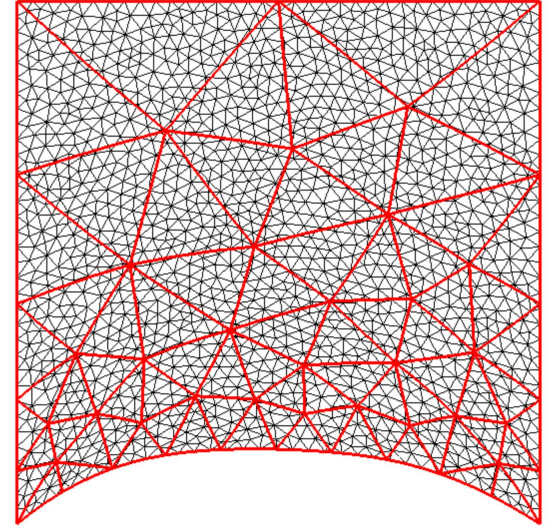

(b) $t=8,0$

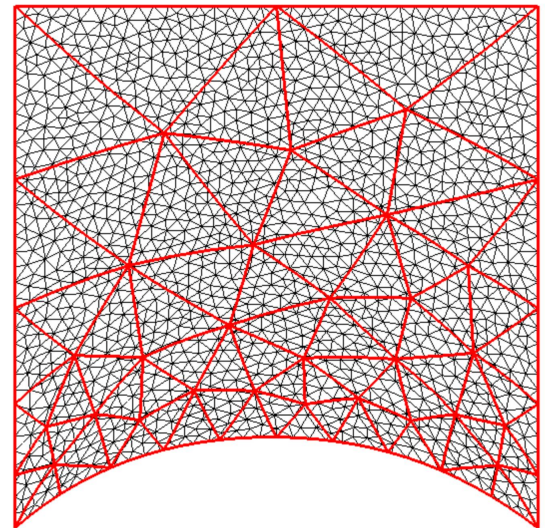

(d) $t=21,0$

Figura 6.9: Configurações deformadas da malha 3 e da malha auxiliar para diversos instantes de tempo. 


\subsubsection{Cavidade tridimensional com fundo flexível}

Neste exemplo, proposto por Mok (2001), a cavidade quadrada é expandida para o caso tridimensional. A estrutura considerada anteriormente é substituída por uma placa quadrada, apoiada nos quatro lados. Na face superior novamente uma velocidade oscilatória é aplicada e quanto às laterais, duas se tratam de paredes com condição de não-escorrecamento e as demais paredes sem atrito. Tanto a geometria quanto as condições de contorno e parâmetros adimensionais do problema são apresentados na Fig. 6.10.

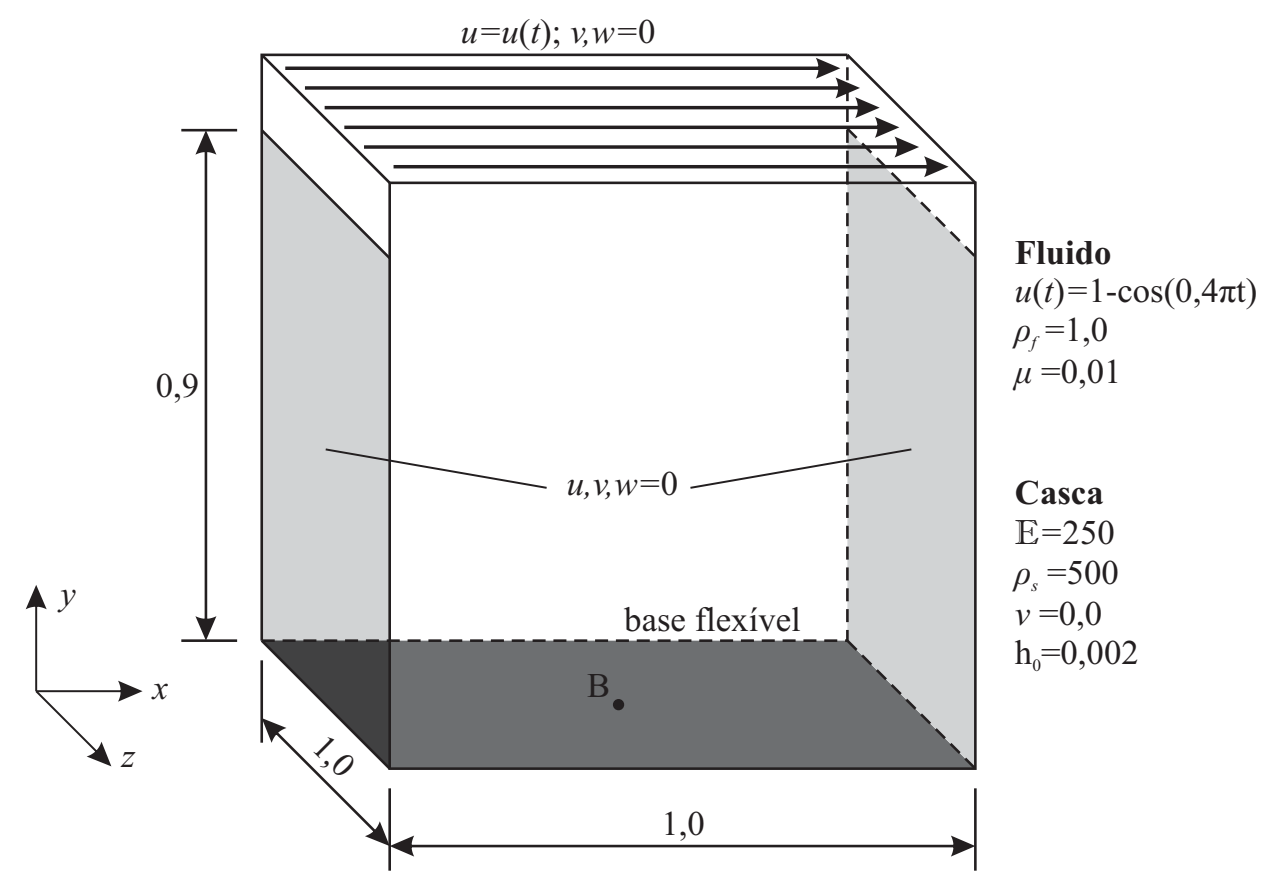

Figura 6.10: Geometria e condições de contorno do problema da cavidade 3D.

A casca que compõe a base da cavidade foi discretizada com uma malha contendo 242 elementos e 1150 nós, como ilustra a Fig. 6.11.

Novamente a velocidade tangencial aplicada à parte superior da cavidade varia entre 0 e 2, implicando num intervalo de 0 a 200 para o Número de Reynolds. Para a discretização do fluido, empregou-se uma malha contendo 10176 elementos com 2364 nós interpolando pressões e 16317 nós interpolando velocidades, ilustrada na Fig. 6.12a. A malha auxiliar, por sua vez conta com 234 elementos e 1459 nós, e é ilustrada na Fig. 6.12b.

Em relação ao fluido, novamente utilizou-se o integrador temporal de primeira ordem com passo de tempo $\Delta t=0,1$, de modo que a Fig. 6.13 apresenta a evolução do deslocamento vertical no ponto $\mathrm{C}$ (centro da casca) com o tempo em comparação com os resultados de Mok (2001) e Vázquez (2007).

Em relação ao deslocamento vertical é possível observar que em comparação ao exemplo anterior, onde tinha-se um escoamento bidimensional, apesar de o comportamento da casca ser muito semelhante, houve um aumento na amplitude de deslocamentos no trecho estacionário de aproximadamente 0,06 para 0,09 . Por outro lado, foram registrados valores menores para a 


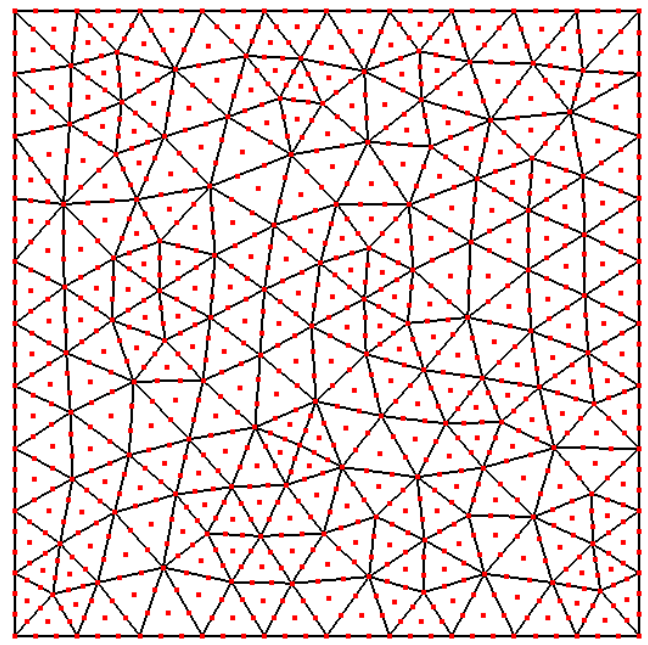

Figura 6.11: Discretização da estrutura.

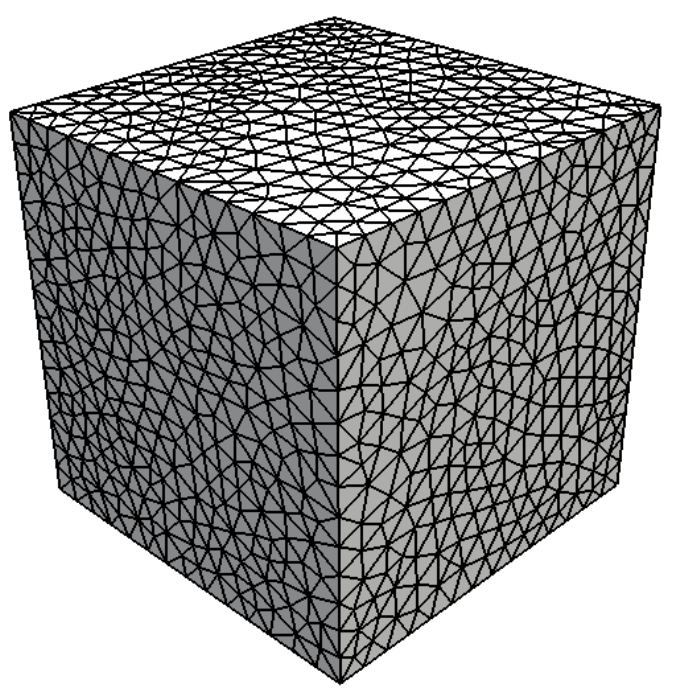

(a) Discretização do fluido.

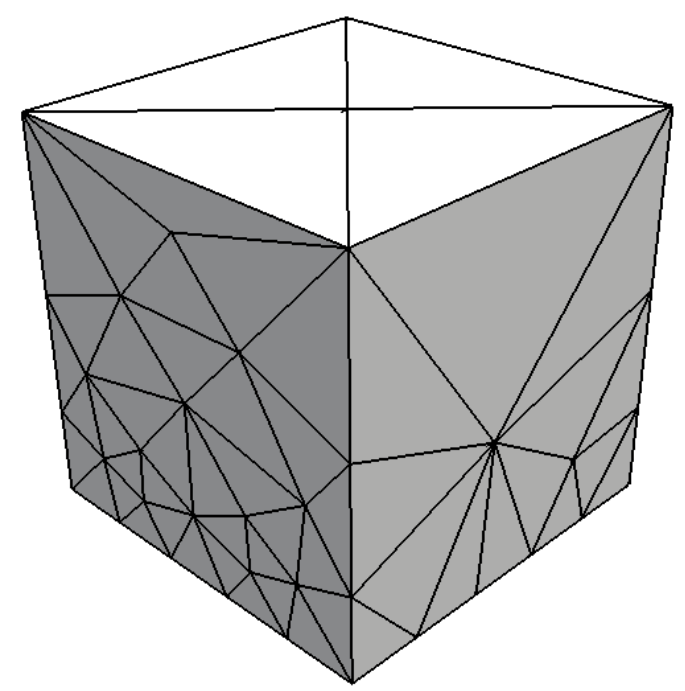

(b) Malha auxiliar.

Figura 6.12: Geometria e condições de contorno do problema da cavidade 3D.

amplitude máxima: 0,188 em relação a 0,212. Isso ocorre devido à restrição de deslocamentos nos quatro lados da casca, que implica em efeitos tridimensionais no escoamento. Contudo, à medida que se tenha uma estrutura mais retangular, maior será a proximidade da resposta com o caso bidimensional e vice-versa.

Quanto à discordância com os resultados das referências, se devem ao fato de ambas não especificarem com precisão todas as condições de contorno aplicadas ao fluido. Mesmo assim, um comportamento parecido foi registrado.

Por fim, na Fig. 6.14 apresentam-se as configurações deformadas da estrutura para diversos instantes de tempo e nas Fig. 6.15 e 6.16, as distribuições dos campos de pressão e velocidade na seção média da cavidade. 


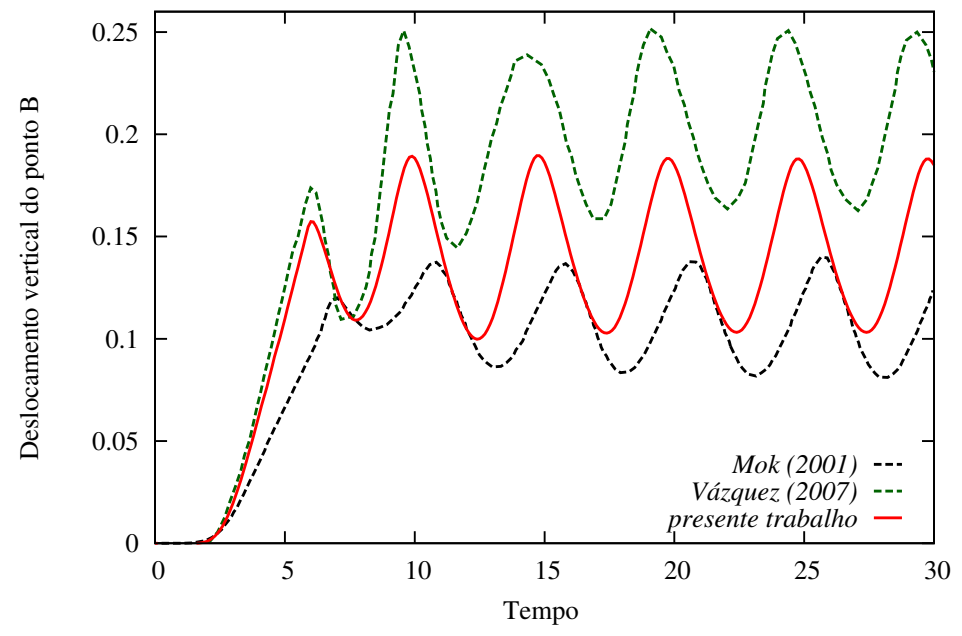

Figura 6.13: Deslocamento vertical do ponto B em função do tempo.

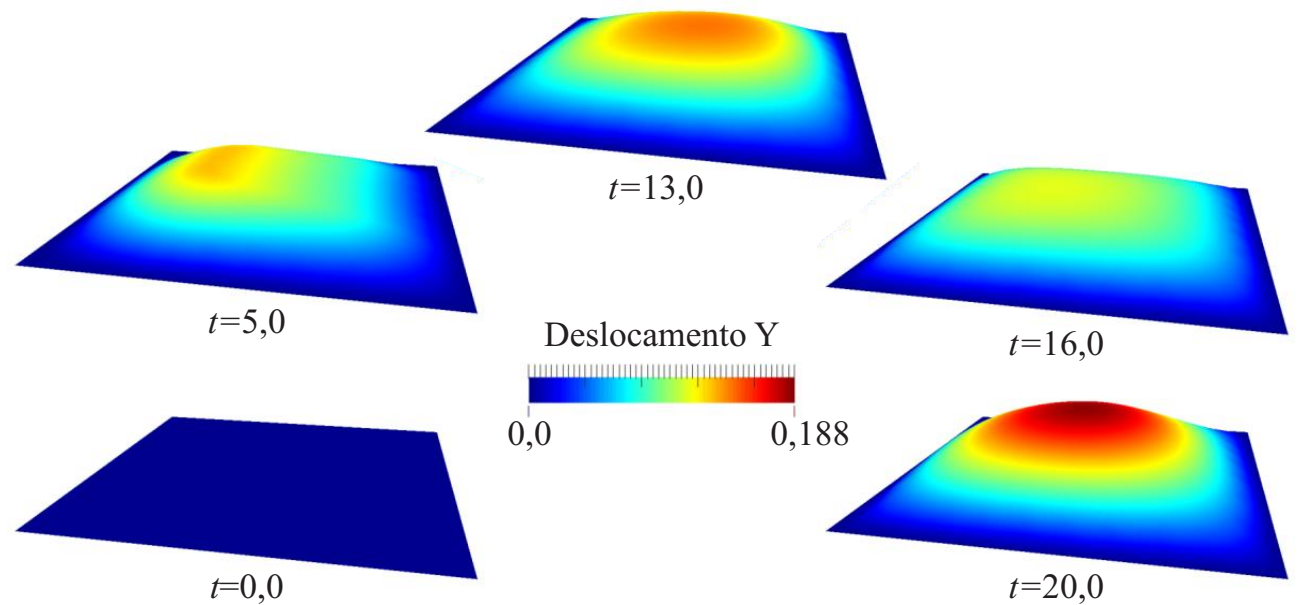

Figura 6.14: Configurações deformadas da base flexível em vários instantes de tempo.

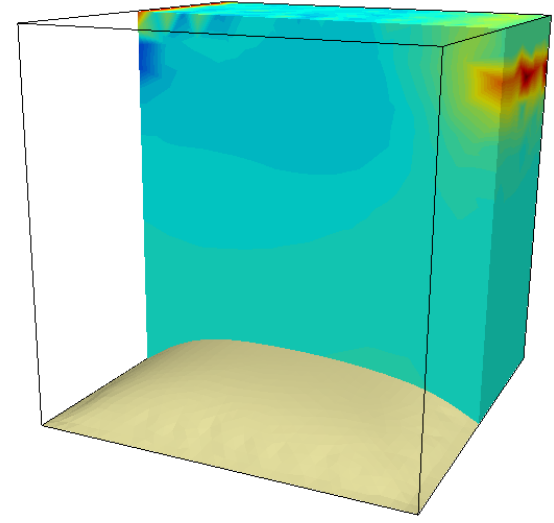

(a) $t=13,0$

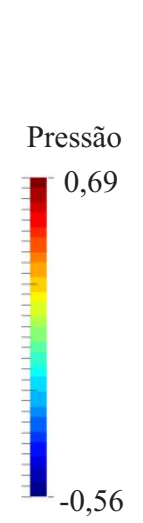

, 56

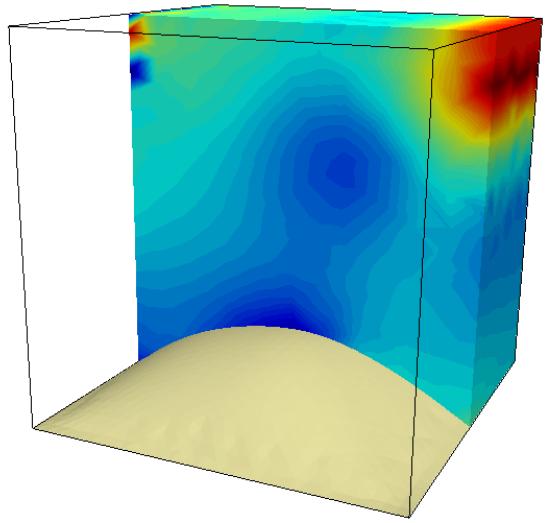

(b) $t=20,0$

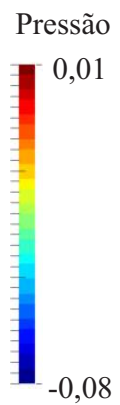

Figura 6.15: Vista da distribuição de pressões na seção central da cavidade. 


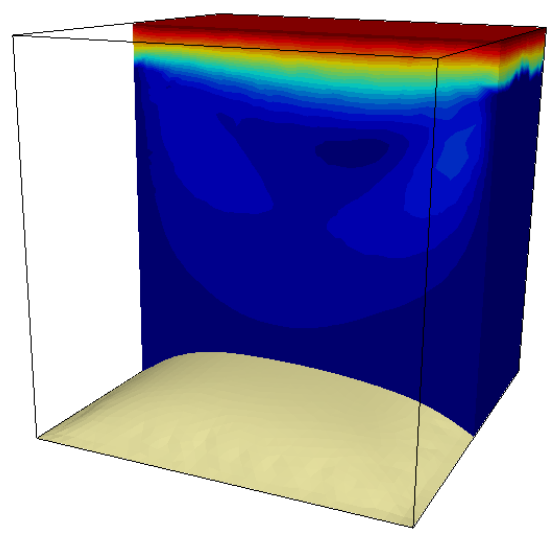

(a) $t=13,0$

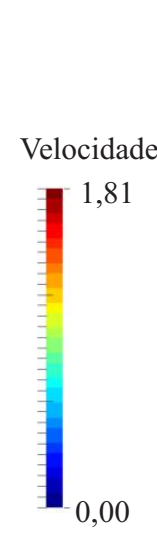

00

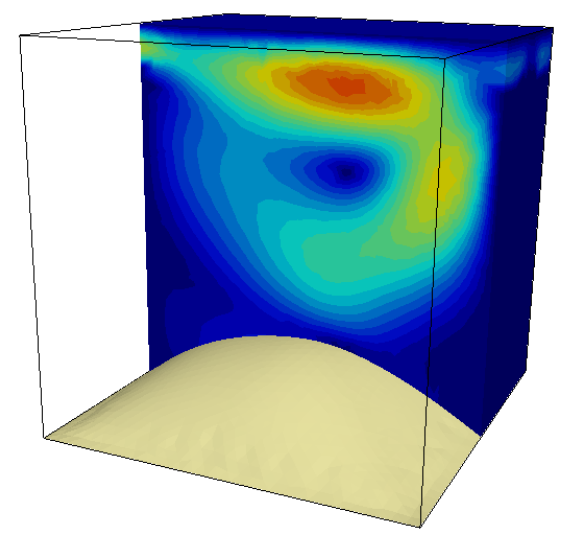

(b) $t=20,0$
Velocidade

T.205

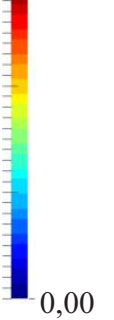

0,00

Figura 6.16: Vista da distribuição de velocidades na seção central da cavidade.

\subsubsection{Painel flexível sob a ação de vórtices gerados por um prisma de seção quadrada}

O problema investigado nesta seção trata-se de um painel engastado a um prisma de seção quadrada, ambos imersos em um meio fluido, como ilustra a Fig. 6.17. O escoamento do fluido sobre o prisma gera vórtices que induzem a estrutura ao desenvolvimento de grandes deslocamentos num fenômeno acoplado de grande complexidade.

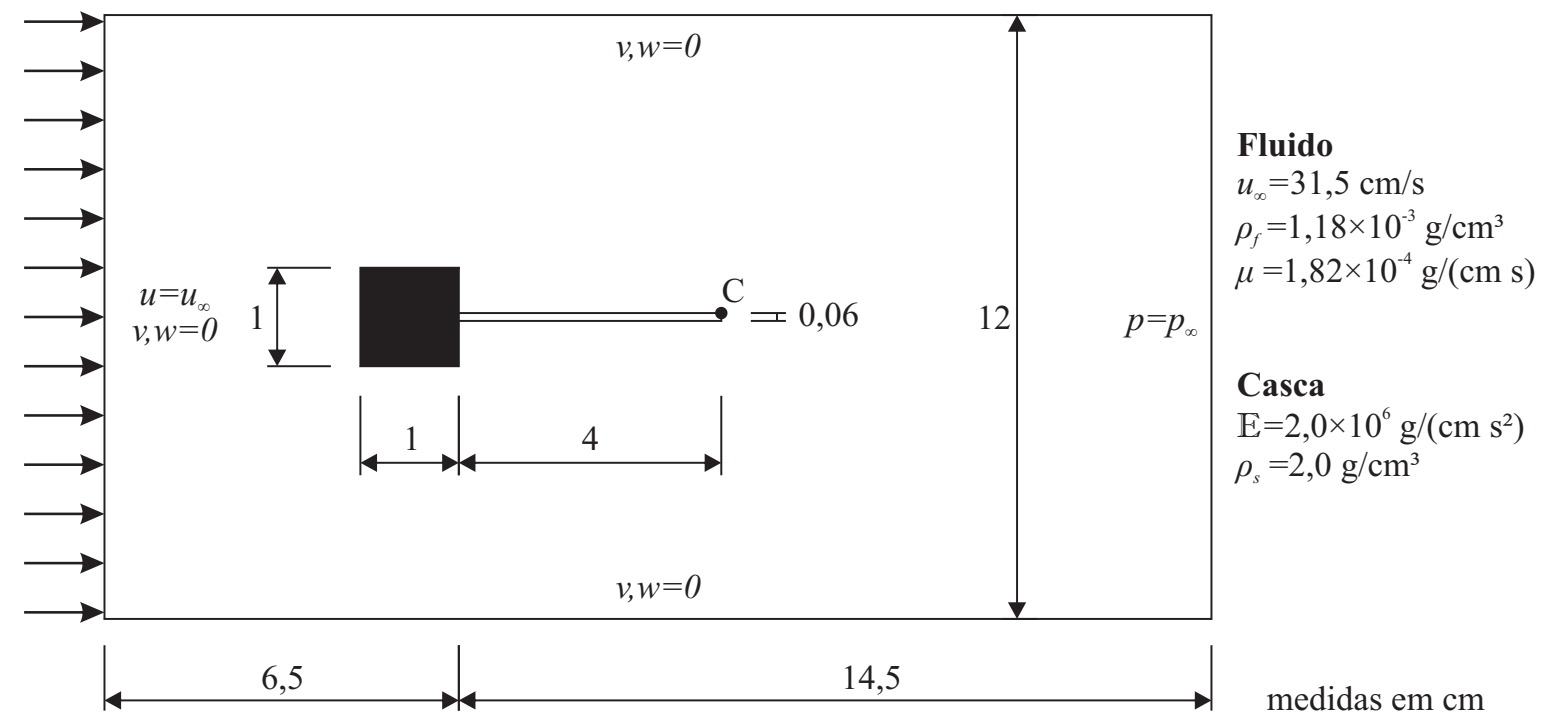

Figura 6.17: Geometria e condições de contorno do problema do painel flexível.

Este exemplo foi inicialmente introduzido por Wall e Ramm (1998) e desde então tem sido utilizado como benchmark para a verificação de códigos de interação fluido-estrutura incompressível em diversos trabalhos. O caso estudado neste trabalho trata-se de uma modificação do problema de Wall proposta por Hübner, Walhorn e Dinkler (2004), cuja velocidade de entrada 
do escoamento é de $31,5 \mathrm{~cm} / \mathrm{s}$, implicando num número de Reynolds de 204 (tomando o lado do prisma como comprimento de referência).

Para efetuar as análises, empregaram-se as malhas apresentadas na Fig. 6.18. A discretização do fluido foi realizada por 8435 elementos, com 2975 nós aproximando pressões e 17341 nós interpolando velocidades. Já a malha auxiliar possui 257 elementos e 1628 nós. Para a representação computacional do painel flexível, usou-se novamente a malha do exemplo 5.5.1, que conta com 40 elementos e 244 nós.

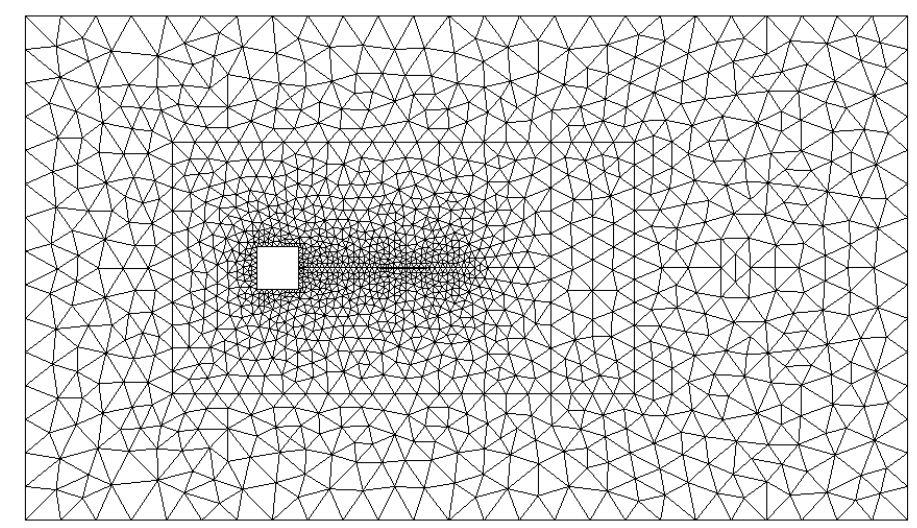

(a) Discretização do fluido.

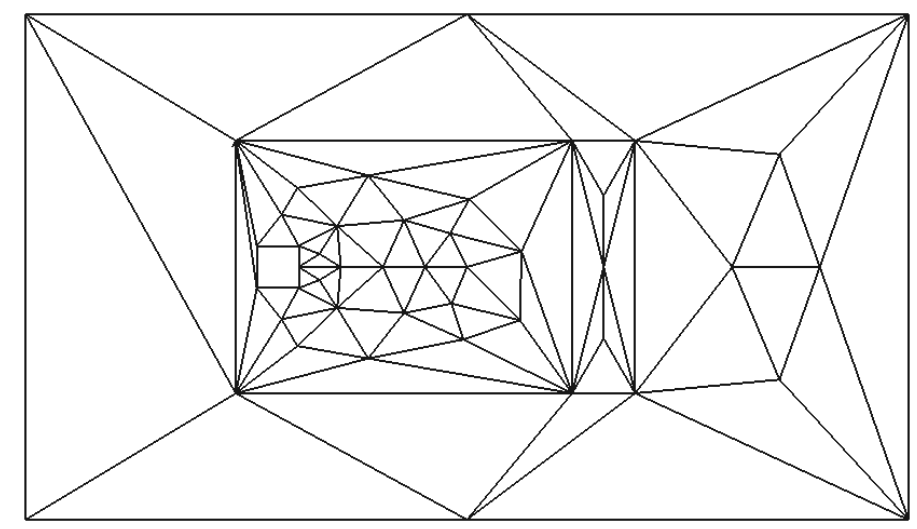

(b) Malha axiliar.

Figura 6.18: Vista frontal das malhas de elementos finitos empregadas na análise do problema do painel flexível.

Para a escolha do passo de tempo, realizou-se um teste preliminar considerando-se a estrutura rígida. Neste caso, verificou-se uma a frequência de desprendimento de vórtices do fluido igual a 3,7 Hz, igual à obtida por Hübner, Walhorn e Dinkler (2004).

Devido à melhor eficiência do integrador de segunda ordem comprovada no exemplo 4.4.3, este modelo de avanço temporal foi aplicado a este exemplo com passo de tempo $\Delta t=$ $5.10^{-4} s$.

Como pode-se observar, o esquema estrutural faz com que a casca neste exemplo tenha comportamento de uma viga engastada. Ao utilizar a teoria clássica de dinâmica das estruturas, como a apresentada por Warburton (1976), tem-se as três primeiras frequências naturais da estrutura iguais a $f^{1}=0,61 \mathrm{~Hz}, f^{2}=3,8 \mathrm{~Hz}$ e $f^{3}=10,6 \mathrm{~Hz}$. 
Desse modo, como a frequência de desprendimento de vórtices do fluido sobre o sistema rígido é muito próximo à segunda frequência natural da estrutura, é esperado que este modo de vibração seja o dominante.

Em relação à estrutura, os nós na região do engaste tiveram todos os seus graus de liberdade restritos e nas demais regiões, apenas as componentes $z$ de posição e vetor generalizado. Para este problema, realizaram-se ainda testes com dois diferentes valores para o coeficiente de Poisson: $v=0,0$ e $v=0,35$, cujo deslocamento vertical da extremidade da estrutura (ponto C) é apresentado na Fig. 6.19 acompanhado da envoltória do resultado obtido por Hübner, Walhorn e Dinkler (2004).

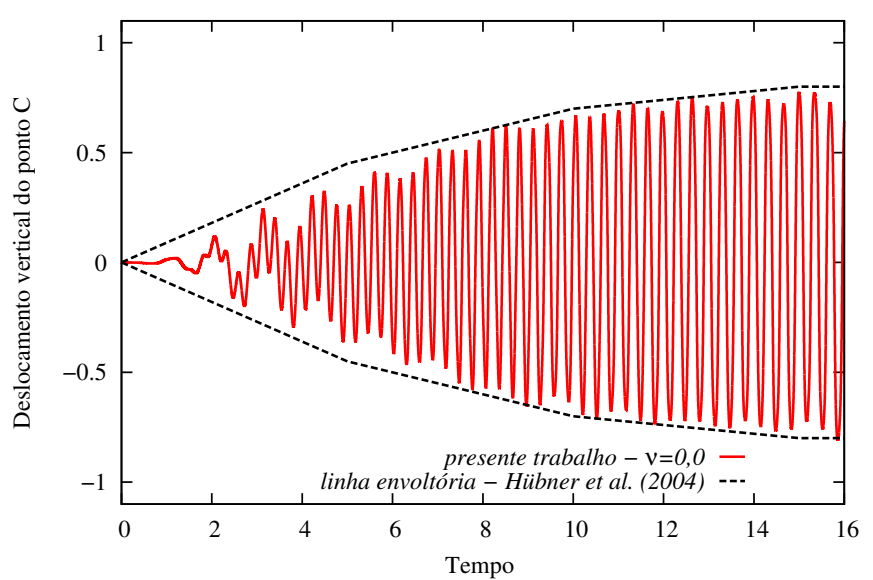

(a) $v=0,0$

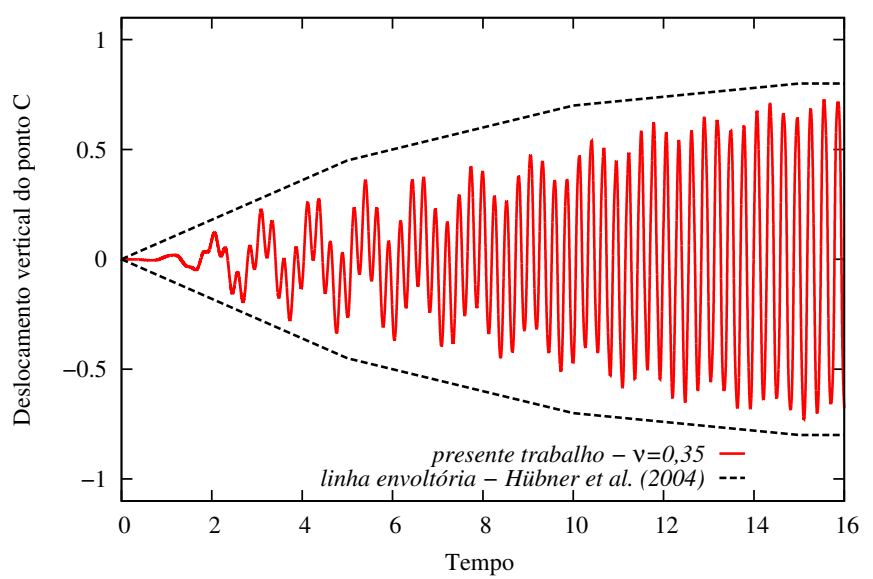

(b) $v=0,35$

Figura 6.19: Deslocamento vertical do ponto C em função do tempo.

Como pode-se perceber, o resultado que mais se aproxima da envoltória é o de $v=0,0$, o que de certo modo é esperado, uma vez que na referência foi utilizado um modelo de viga de Euler-Bernoulli que, diferentemente da cinemática de Reissner, não considera deformações por cisalhamento na estrutura.

Outra constatação é que a estrutura de fato teve o segundo modo de vibração como dominante, entretanto a frequência do problema acoplado foi de $3,05 \mathrm{~Hz}$ para $v=0,0$ e $3,47 \mathrm{~Hz}$ 
para $v=0,35$. Esse resultado encontra-se novamente em pleno acordo com a referência, que obteve uma frequência de 3,1 Hz para o fenômeno acoplado.

Quanto à amplitude de deslocamentos, os resultados também foram muito próximos à referência, variando entre o intervalo de $-0,8 \mathrm{~cm}$ e $0,8 \mathrm{~cm}$ para o caso de $v=0,0$. Já para $v=0,35$, o intervalo de deslocamentos obtido gira em torno de $-0,75 \mathrm{~cm}$ a $0,75 \mathrm{~cm}$.

Por fim, tomou-se um ciclo da resposta estacionária obtida para $v=0,0$, cujo período é igual a $T$. Sendo assim, as Fig. 6.20, 6.21 e 6.22 apresentam os perfis de velocidade e pressão, bem como a configuração deformada da malha do fluido para seis instantes de tempo pertencentes a este ciclo do movimento.

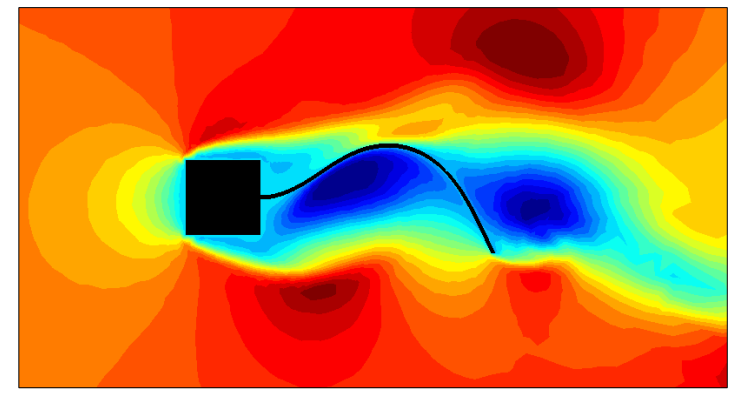

(a) $t=n T$

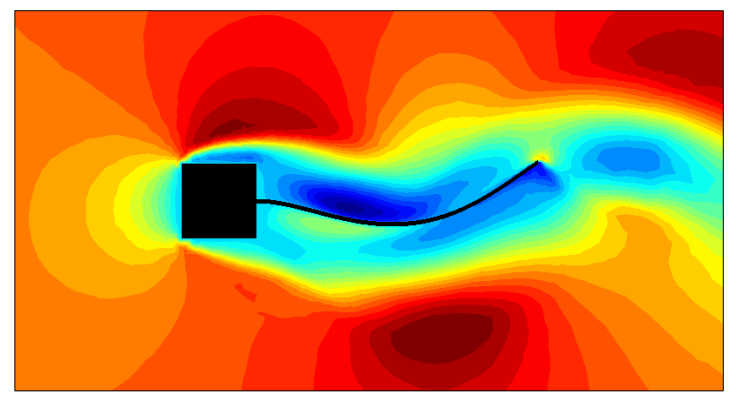

(c) $t=n T+\frac{2 T}{6}$

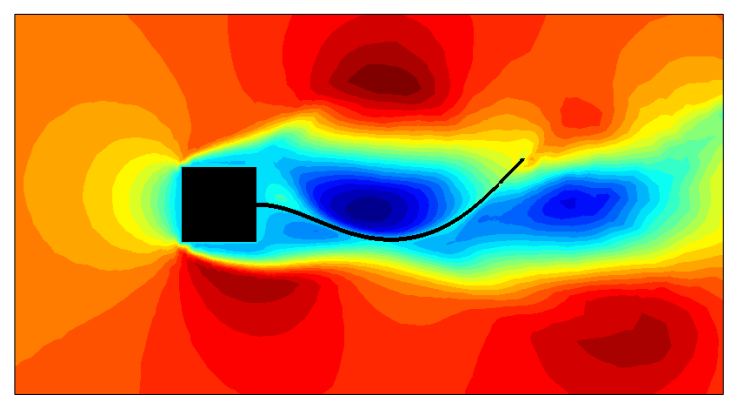

(e) $t=n T+\frac{4 T}{6}$

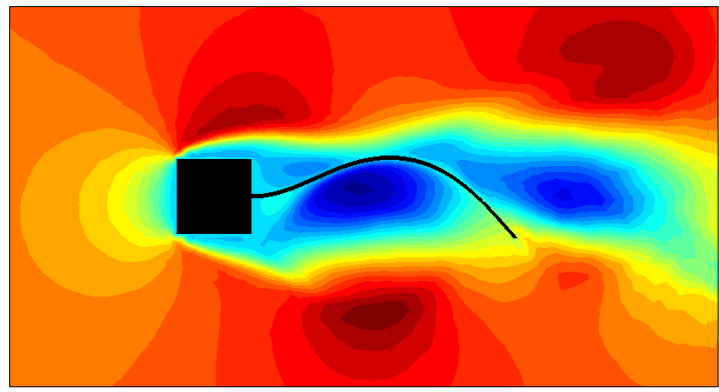

(b) $t=n T+\frac{T}{6}$

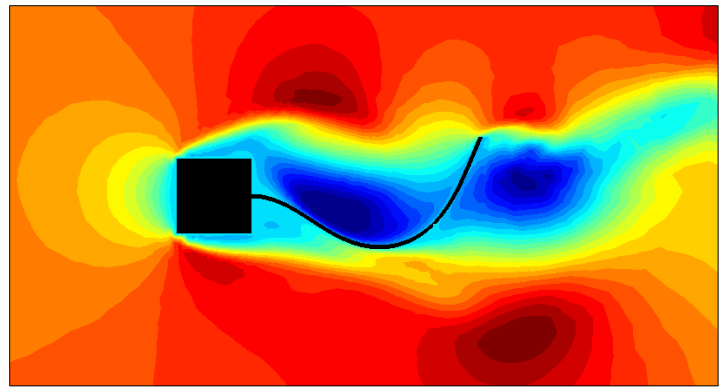

(d) $t=n T+\frac{3 T}{6}$

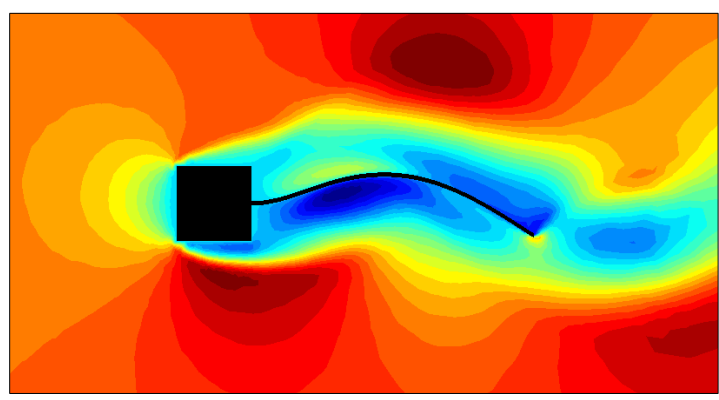

(f) $t=n T+\frac{5 T}{6}$

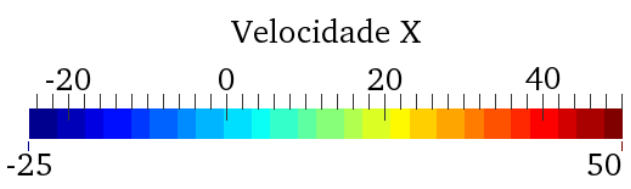

Figura 6.20: Distribuição da componente $X$ de velocidade em diferentes instantes de tempo. 


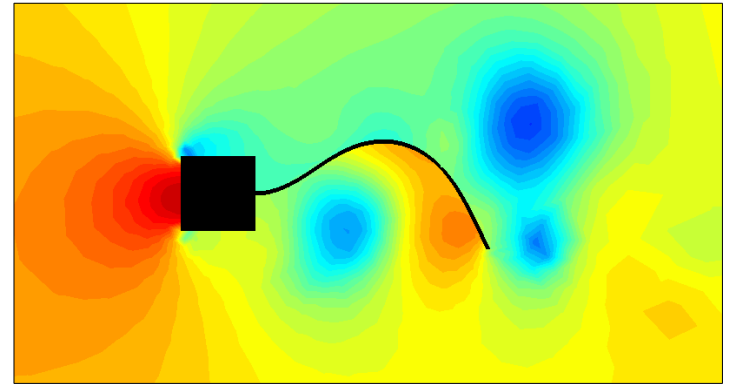

(a) $t=n T$

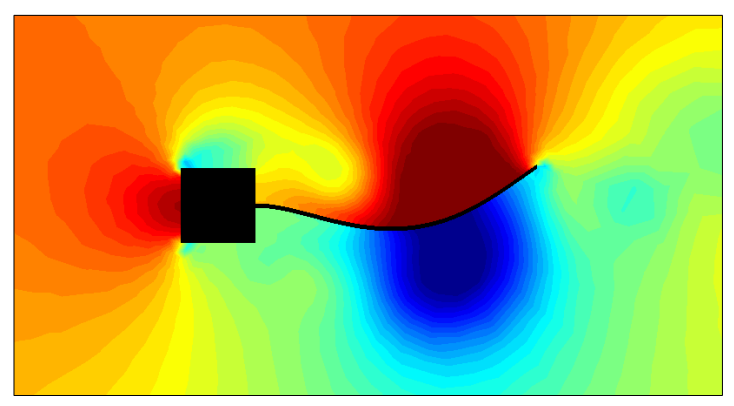

(c) $t=n T+\frac{2 T}{6}$

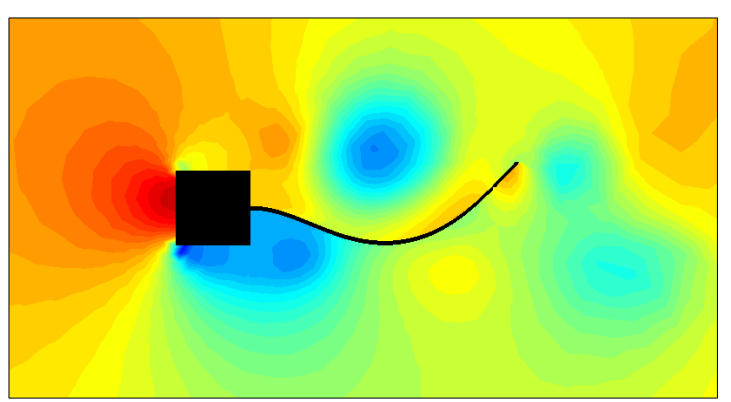

(e) $t=n T+\frac{4 T}{6}$

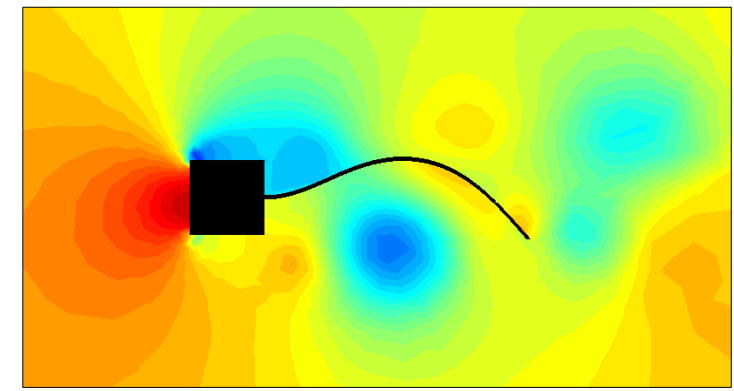

(b) $t=n T+\frac{T}{6}$

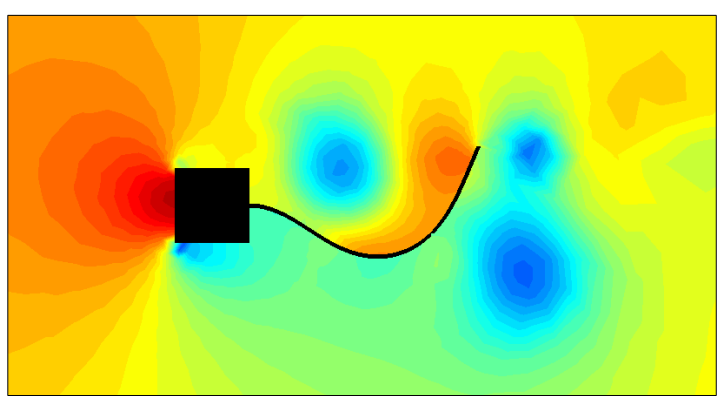

(d) $t=n T+\frac{3 T}{6}$

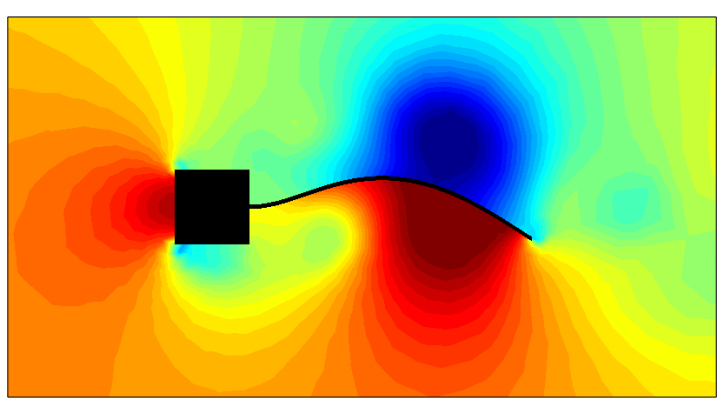

(f) $t=n T+\frac{5 T}{6}$

Pressão

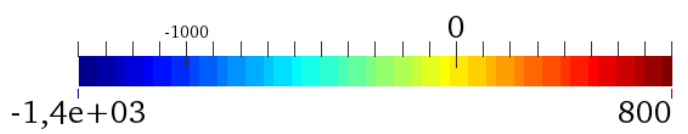

Figura 6.21: Distribuição de pressão em diferentes instantes de tempo. 


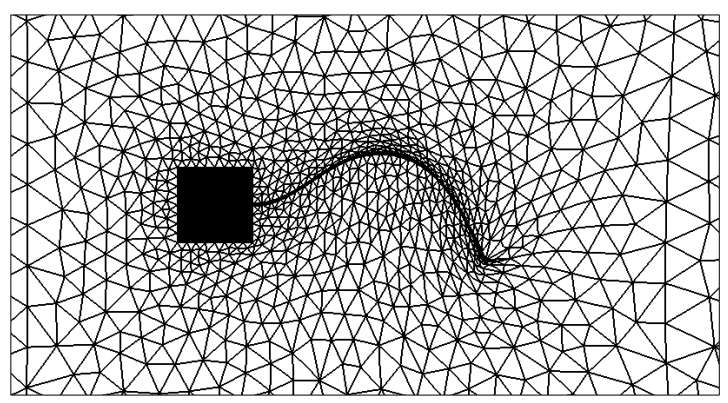

(a) $t=n T$

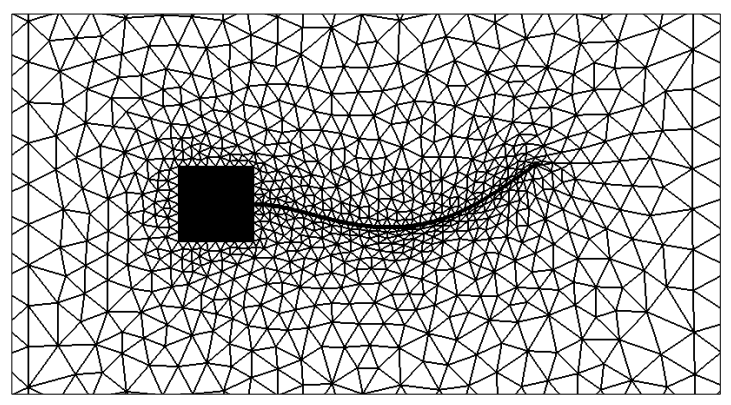

(c) $t=n T+\frac{2 T}{6}$

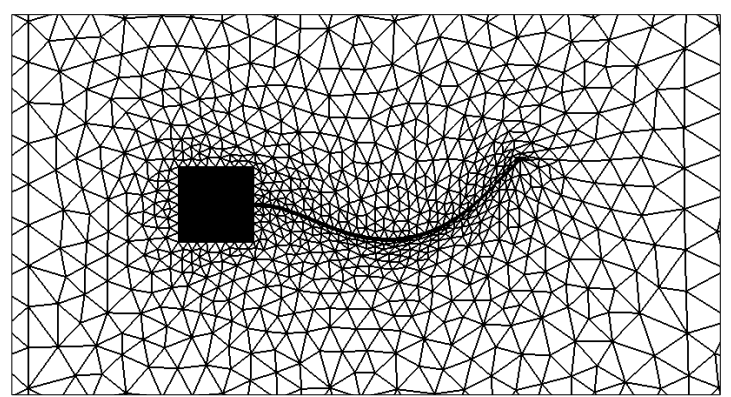

(e) $t=n T+\frac{4 T}{6}$

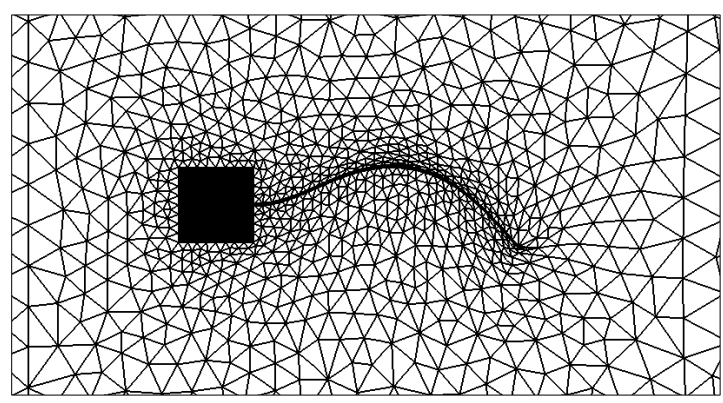

(b) $t=n T+\frac{T}{6}$

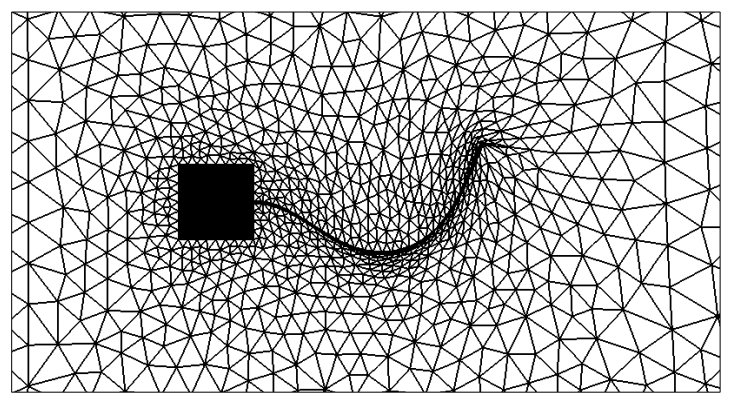

(d) $t=n T+\frac{3 T}{6}$

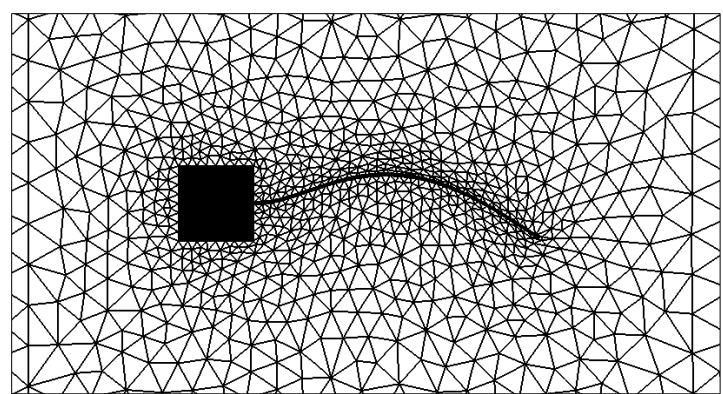

(f) $t=n T+\frac{5 T}{6}$

Figura 6.22: Configuração deformada da malha do fluido na região da interface com a estrutura em diferentes instantes de tempo. 


\section{CAPÍtULO}

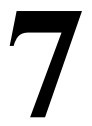

\section{CONCLUSÃO}

Neste trabalho uma abordagem pelo MEF posicional para a modelagem de cascas dinâmicas considerando a não linearidade geométrica foi acoplada a uma metodologia com base no MEF para a simulação de fluidos newtonianos em descrição ALE com o emprego de elementos finitos de ordem quadrática para velocidade e linear para pressão. Além disso, uma proposta de acoplamento sem a necessidade de coincidência dos nós dos domínios acompanhada de um esquema de movimentação dinâmica da malha do fluido baseada na utilização de uma malha auxiliar com elementos de ordem cúbica foi implementada com sucesso.

O tratamento do fluido por meio da formulação mista, ou velocidade-pressão, com estabilização por meio da técnica SUPG se mostrou bastante apropriada para a simulação de escoamentos laminares, produzindo resultados satisfatórios e em conformidade com a literatura.

Para a consideração da não linearidade geométrica das estruturas de casca, uma formulação descrita em posições e que não interpola giros como graus de liberdade foi empregada. Esta técnica se mostrou robusta e capaz de simular problemas de instabilidade dinâmica, tais como o exemplo 5.5.2..

As dificuldades em trabalhar com descrições matemáticas distintas para os problemas físicos (Lagrangeana para o sólido e Euleriana para o fluido) foram superadas pela adoção de uma descrição Lagrangeana-Euleriana Arbitrária para o fluido, permitindo que seu domínio computacional tenha movimento independente e arbitrário.

Quanto ao acoplamento fluido-estrutura, a compatibilização de deslocamentos entre os meios deve ser realizada. O método adotado (baseado na descrição ALE) exige a movimentação da malha do fluido, o que no caso tridimensional pode ser bastante custoso computacionalmente. Nesse sentido, um esquema de movimentação do domínio computacional a partir de uma malha auxiliar foi introduzido, mostrando-se efetivo tanto pela qualidade dos resultados obtidos quanto pela redução do número de graus de liberdade do problema a ser resolvido. Cabe destacar que o emprego da malha auxiliar torna o processo de movimentação da malha do fluido menos suscetível à instabilidades causadas pela utilização de um método de suavização Laplaciana, 
como problemas relacionados à distorções excessivas ou inversão de elementos próximos à interface fluido-estrutura.

O modelo de acoplamento particionado fraco em seu esquema clássico Dirichlet-Neumann foi implementado e submetido a testes envolvendo grandes deslocamentos da estrutura, apresentando resultados coerentes com as referências tomadas.

Cabe mencionar que ao buscar a simulação de problemas em que a densidade do fluido e da estrutura em muito próximas, não foram alcançadas respostas estáveis, o que é esperado em métodos particionados e atribuído ao efeito de massa adicionada. Para que estes casos possam ser estudados com a metodologia deste trabalho, devem ser implementados avanços no esquema de acoplamento, tais como o emprego de condições de Robin para a transferência de informações entre os meios, ou ainda técnicas de acoplamento particionado forte.

Entretanto, dada a complexidade dos casos simulados, principalmente do exemplo 6.4.3, comprova-se a eficiência e robustez da metodologia utilizada.

Para que se torne factível a simulação de problemas tridimensionais com maior precisão, o código computacional deve ser expandido aplicando-se técnicas mais elaboradas de programação paralela. Além disso, métodos mais eficientes para a resolução sistema não linear advindo da formulação mista aplicada ao fluido devem ser estudados. Nesse sentido melhorias significativas vêm sendo reportadas na literatura, tal como a utilização de métodos iterativos como o algoritmo de Uzawa e o GMRES (Generalized Minimal Residual Method) aliados à precondicionadores do sistema de equações.

Para o avanço da pesquisa, sugere-se o estudo de interação fluido-estrutura incompressível com técnicas sem movimentação da malha do fluido, como as de contorno imerso. Além disso, propõe-se o estudo de técnicas alternativas para o tratamento do problema de fluido, tais como os métodos multiescala (VMS), de partículas finitas (PFEM) e de Galerkin descontínuo, além de modelos de turbulência para a simulação de problemas com elevado número de Reynolds. 
AKIN, T. E. T. J. E. Calculation of the advective limit of the supg stabilization parameter for linear and higher-order elements. Computer Methods in Applied Mechanics and Engineering, v. 193, n. 21-22, p. 1909-1922, 2004.

ANDERSON, J. D. Computational fluid dynamic - the basics with applications. 1. ed. New York, USA: McGraw-Hill Book Company, 1995.

ARGYRIS, J. An excursion into large rotations. Computer Methods in Applied Mechanics and Engineering, v. 32, p. 85-155, 1982.

ARGYRIS, J.; BALMER, H.; DOLTSINIS, J. S.; DUNNE, P. C.; HAASE, M.; KLEIBER, M.; MALEJANNAKIS, G. A.; MLEJNEK, H. P.; MÜLLER, M.; SCHARPF, D. W. Finite element method - the natural approach. Computer Methods in Applied Mechanics and Engineering, v. 17-18, n. 1, p. 1-106, 1979.

ARGYRIS, J.; PAPADRAKAKIS, M.; MOUROUTIS, Z. S. Nonlinear dynamic analysis of shells with the triangular element tric. Comput. Methods Appl. Mech. Engrg., v. 192, p. 3005-3038, 2003.

ARGYRIS, J. H.; DUNNE, P. C.; MALEJANNAKIS, G.; SCHARPF, D. W. On large displacement-small strain analysis of structures with rotational degrees of freedom. Computer Methods in Applied Mechanics and Engineering, v. 14, n. 3, p. 401-451, 1978.

BADIA, S.; NOBILE, F.; VERGARA, C. Fluid-structure partitioned procedures based on robin transmission conditions. Journal of Computational Physics, v. 227, p. 7027-7051, 2008.

BATHE, K. J. Finite element procedures in engineering analysis. 2. ed. New Jersey: Prentice-Hall, 1996.

BATHE, K. J.; RAMM, E.; WILSON, E. L. Finite element formulations for large deformation dynamic analysis. International Journal for Numerical Methods in Engineering, v. 9, p. 353-386, 1975.

BATTINI, J. M.; PACOSTE, C. On the choice of the linear element for corotational triangular shells. Computer Methods in Applied Mechanicas and Engineering, v. 195, n. 44-47, p. 6362-6377, 2006. ISSN 0045-7825.

BAZILEVS, Y.; CALO, V. M.; COTTRELL, J. A.; HUGHES, T. J. R.; REALI, A.; SCOVAZZI, G. Variational multiscale residual-based turbulence modeling for large eddy simulation of incompressible flows. Comput. Methods Appl. Mech. Engrg., v. 197, p. 173-201, 2007.

BAZILEVS, Y.; KOROBENKO, A.; YAN, J. Ale-vms formulation for stratified turbulent incompressible flows with applications. Mathematical Models and Methods in Applied Sciences, v. 25, p. 2349-2375, 2015.

BAZILEVS, Y.; TAKIZAWA, K.; TEZDUYAR, T. E. Computational Fluid-Structure Interaction: Methods and Applications. Chichester, UK: John Wiley \& Sons, 2013. 
BELO, I. M. Desenvolvimento da formulação corrotacional em elementos finitos de casca para análise hiperelástica. Tese (Doutorado) — UFSC, Florianópolis, Santa Catarina, Brasil, 2009.

BELYTSCHKO, T.; SCHWER, L.; KLEIN, M. J. Large displacement, transient analysis of space frames. International Journal for Numerical Methods in Engineering, v. 11, n. 1, p. 65-84, 1977. ISSN 0029-5981.

BENZI, M.; GOLUB, G. H.; LIESEN, J. Numerical solution of saddle point problems. Acta Numerica, v. 14, p. 1-137, 2005.

BLOM, F. J. A monolithical fluid-structure interaction algorithm applied to the piston problem. Computer Methods in Applied Mechanics and Engineering, v. 167, n. 3-4, p. 369-391, 1998. ISSN 0045-7825.

BOFFI, D.; GASTALDI, L. Stability and geometric conservation laws for ale formulations. Comput. Methods Appl. Mech. Engrg., v. 193, p. 4717-4739, 2004.

BONET, J.; WOOD, R. D. Nonlinear continuum mechanics for finite element analysis. 2. ed. United Kingdom: Cambridge University Press, 2008.

BONET, J.; WOOD, R. D.; MAHANEY, J.; HEYWOOD, P. Finite element analysis of air supported membrane structures. Computer Methods in Applied Mechanics and Engineering, v. 190, n. 5-7, p. 579-595, 2000. ISSN 0045-7828.

BRENDEL, B.; RAMM, E. Linear and nonlinear stability analysis of cylindrical shells. Computers \& Structures, v. 12, n. 4, p. 549-558, October 1980.

BROOKS, A. N.; HUGHES, T. J. Streamline upwind/petrov-galerkin formulations for convection dominated flows with particular emphasis on the incompressible navier-stokes equations. Computer Methods in Applied Mechanics and Engineering, v. 32, n. 1-3, p. 199-259, 1982.

BRUMMELEN, E. H. V. Added mass effects of compressible and incompressible flows in fluid-structure interaction. Journal of Applied Mechanics, v. 79, n. 021206, 2009.

BURMAN, E.; FERNÁNDEZ, M. A. Explicit strategies for incompressible fluid-structure interaction problems: Nitsche type mortaring versus robin-robin coupling. Int. J. Numer. Meth. Engrg., v. 97, p. 739-758, 2014.

CATABRIGA, L.; COUTINHO, A. L. G. Implicit supg solution of euler equations using edge-based data structures. Computer Methods in Applied Mechanics and Engineering, v. 191, n. 32, p. 3477-3490, 2002. ISSN 0045-7825.

CAUSIN, P.; GERBEAU, J. F.; NOBILE, F. Added-mass effect in the design of partitioned algorithms for fluid-structure problems. Computer Methods in Applied Mechanics and Engineering, v. 194, p. 42-44, 2005. ISSN 4506-4527.

CHUNG, T. J. Computional fluid dynamics. Cambridge, UK: Cambridge University Press, 2002.

CODA, H. B. Análise não linear geométrica de sólidos e estruturas: Uma formulação posicional baseada no MEF. Tese (Tese para concurso de professor titular) — SET-EESC-USP, São Carlos, São Paulo, Brasil, 2003. 
CODA, H. B. A solid-like fem for geometrically non-linear 3d frames. Computer Methods in Applied Mechanics and Engineering, v. 198, n. 47-48, p. 3712-3722, 2009a.

CODA, H. B. Two dimensional analysis of inflatable structures by the positional fem. Latin American Journal of Solids and Structures, v. 6, n. 3, p. 187-212, Sep 2009b. ISSN 1679-7817.

CODA, H. B.; PACCOLA, R. R. An alternative positional fem formulation for geometrically non-linear analysis of shells: Curved triangular isoparametric elements. Computional Mechanics, v. 40, n. 1, p. 185-200, jun 2007.

CODA, H. B.; PACCOLA, R. R. A positional fem formulation for geometrical non-linear analysis of shells. Latin American Journal of Solids and Structures, v. 5, p. 205-223, 2008.

CODA, H. B.; PACCOLA, R. R. Unconstrained finite element for geometrical nonlinear dynamics of shells. Mathematical Problems in Engineering, 2009. ISSN 1024-123X.

CODA, H. B.; PACCOLA, R. R. Improved finite element for 3d laminate frame analysis including warping for any cross-section. Applied Mathematical Modelling, v. 34, n. 4, p. 1107-1137, 2010.

CODINA, R.; COPPOLA-OWEN, H.; NITHIARASU, P.; LIU, C. B. Numerical comparison of cbs and sgs as stabilization techniques for the incompressible navier-stokes equations. International Journal for Numerical Methods in Engineering, v. 66, p. 1672-1689, 2006.

COLLAR, A. R. The expanding domain of aeroelasticity. Journal of the Royal Aeronautical Society, 1946.

CRISFIELD, M. A. Non-linear finite element analysis of solid and structures. England: John Wiley \& Sons, 1991.

CUNHA, F. S. Apostila do curso Estruturas Aeroespaciais. Lisboa: Universidade Técnica de Lisboa, 2004.

DÁVALOS, C.; CANTE, J.; HERNÁNDEZ, J. A.; OLIVER, J. On the numerical modeling of granular material flows via the particle finite element method (pfem). International Journal of Solids and Structures, v. 71, p. 99-125, 2015.

DING, H.; SHU, C.; YEO, K. S.; XU, D. Simulation of incompressible viscous flows past a circular cylinder by hybrid fd scheme and meshless least square-based finite difference method. Comput. Methods Appl. Mech. Engrg., v. 193, p. 727-744, 2004.

DONEA, J.; GIULIANI, S.; HALLEUX, J. P. An arbitrary lagrangian-eulerian finite element method for transient dynamic fluid-structure interactions. Computer Methods in Applied Mechanics and Engineering, v. 33, n. 1-3, p. 689-723, 1982.

DONEA, J.; HUERTA, A. Finite Element Methods for flow problems. Chichester, UK: John Wiley \& Sons, 2003.

DOWELL, E. H. A Modern Course in Aeroelasticity. 5. ed. Heidelberg: Springer, 2015.

ELMAN, H. C.; SILVESTER, D. J.; WATHEN, A. J. Block preconditioners for the discrete incompressible navier-stokes equations. Int. J. Numer. Meth. Fluids, v. 40, p. 333-344, 2002.

ELMAN, H. C.; SILVESTER, D. J.; WATHEN, A. J. Finite Elements and Fast Iterative Solvers: with Applications in Incompressible Fluid Dynamics. New York: Oxford, 2005. 
FARHAT, C.; LESOINNE, M.; MAMAN, N. Mixed explicit/implicit time integration of coupled aeroelastic problems: three-field formulation, geometric conservation and distributed solution. Int. J. Numer. Meth. Fluids, v. 21, p. 807-835, 1995.

FELIPPA, C. A.; PARK, K. C.; FARHAT, C. Partitioned analysis of coupled mechanical systems. Computer Methods in Applied Mechanics and Engineering, v. 190, n. 24-25, p. 3247-3270, 2001.

FORMAGGIA, L.; NOBILE, F. Stability analysis of second-order time accurate schemes. Comput. Methods Appl. Mech. Engrg., v. 193, p. 4097-4116, 2004.

FÖRSTER, C.; WALL, W. A.; RAMM, E. Artificial added mass instabilities in sequential staggered coupling on nonlinear structures and incompressible viscous flows. Comput. Methods Appl. Mech. Engrg., v. 196, p. 1278-1293, 2007.

GERBEAU, J. F.; VIDRASCU, M. A quasi-newton algorithmm based on a reduced model for fluid-structure interaction problems in blood flows. ESAIM: Mathematical Modelling and Numerical Analysis, v. 37, p. 631-647, 2003.

GHIA, U.; GHIA, K. N.; SHIN, C. T. High-re solutions for incompressible flow using the navier-stokes equations and a multigrid method. Journal of Computational Physics, v. 48, p. 387-441, 1982.

GINGOLD, R. A.; MONAGHAN, J. J. Smoothed particle hydrodynamics, theory and application to non-spherical stars. Monthly Notices of the Royal Astronomical Society, v. 181, p. 375-389, 1997.

GLOWINSKI, R. Finite Element Methods for Incompressible Viscous Flow. Amsterdam, NL: Elsevier Science, B.V., 2003.

GRESHO, P. M.; CHAN, S. T.; LEE, R. L.; UPSON, C. D. A modified finite element method for solving the time-dependent, incompressible navier-stokes equations. part 2: Applications. International Journal for Numerical Methods in Fluids, v. 4, p. 619-640, 1984.

HEIL, M.; HAZEL, A. L.; BOYLE, J. Solvers for large-displacement fluid-structure interaction problems: segregated versus monolithic approaches. Comput. Mech., v. 43, p. 91-101, 2008.

HIRSCH, G. H. Damping measures to control wind-induced vibrations. In: SOCKEL, H. (Ed.). Wind-excited vibrations of structures, CISM Course. Wien-New York: Springer-Verlag, 1994. p. $1-51$.

HODGES, D. H.; PIERCE, G. A. Introduction to Structural Dynamics and Aeroelasticity. 2. ed. New York: Cambridge University Press, 2011.

HOU, G.; WANG, J.; LAYTON, A. Numerical methods for fluid-structure interaction - a review. Commun. Comput. Phys., v. 12, p. 337-377, 2012.

HRON, J.; MADLIK, M. Fluid-structure interaction with applications in biomechanics. Nonlinear Analysis: Real World Applications, v. 8, n. 5, p. 1431-1458, 2007.

HÜBNER, B.; WALHORN, E.; DINKLER, D. A monolithic approach to fluid-structure interaction using space-time finite elements. Comput. Methods Appl. Mech. Engrg., v. 193, p. 2087-2104, 2004. 
HUGHES, T. J.; CARNOY, E. Nonlinear finite element shell formulation accounting for large membrane strains. Computer Methods in Applied Mechanics and Engineering, v. 39, n. 1, p. 69-82, 1983.

HUGHES, T. J. R. Multiscale phenomena: Green's functions, the dirichlet-to-neumann formulation, subgrid scale methods, bubbles and the origins of stabilized methods. Comput. Methods Appl. Mech. Engrg, v. 127, p. 387-401, 1995.

HUGHES, T. J. R.; FEIJÓO, G. R.; MAZZEI, L.; QUINCY, J. B. The variational multiscale method - a paradimg for computational mechanics. Comput. Methods Appl. Mech. Engrg, v. 166, p. 3-24, 1998.

HUGHES, T. J. R.; LIU, W. K. Nonlinear finite element analysis of shells: Part i.

three-dimensional shells. Computer Methods in Applied Mechanics and Engineering, v. 26, n. 3, p. 331-362, 1981. A. ISSN 0045-7825.

HUGHES, T. J. R.; LIU, W. K. Nonlinear finite element analysis of shells: Part ii. two-dimensional shells. Computer Methods in Applied Mechanics and Engineering, v. 27, n. 2, p. 167-181, 1981. A. ISSN 0045-7825.

HUGHES, T. J. R.; MAZZEI, L.; JANSEN, K. E. Large eddy simulation and the variational multiscale method. Comput. Visual Sci, v. 3, p. 47-59, 2000.

HUGHES, T. J. R.; OBERAI, A. A.; MAZZEI, L. Large eddy simulation of turbulent channel flows by the variational multiscale method. Physics of Fluids, v. 13, p. 1874-1799, 2001.

IBRAHIMBEGOVIC, A.; TAYLOR, R. L. On the role of frame-invariance in structural mechanics models at finite rotations. Comput. Methods Appl. Mech. Engrg., v. 191, p. 5159-5176, 2002.

IDELSOHN, S. R.; MARTI, J.; LIMACHE, A.; OÑATE, E. Unified lagrangian formulation for elastic solids and incompressible fluids: Application to fluid-structure interaction problems via the pfem. Comput. Methods Appl. Mech. Engrg., v. 197, p. 1792-1776, 2008.

IDELSOHN, S. R.; OÑATE, E.; PIN, F. D. The particle finite element method: a powerful tool to solve incompressible flows with free-surfaces and breaking waves. Int. J. Numer. Meth. Engrg., v. 61, p. 964-989, 2004.

JOHN, V.; KAYA, S. A finite element variational multiscale method for the navier-stokes equations. SIAM J. Sci. Comput., v. 26, p. 1485-1503, 2005.

KANCHI, H.; MASUD, A. A 3d adaptative mesh moving scheme. International Journal for Numerical Methods in Fluids, v. 54, p. 923-944, 2007.

KAWABATA, C. L. O.; NETO, M. K.; CODA, H. B.; VENTURINI, W. S. Desenvolvimento e implementação de um método de elementos finitos paralelo para análise não linear de estruturas. In: XXX Congresso Íbero Latino Americano de Métodos Computacionais em Engenharia CILAMCE. Búzios, Brasil: [s.n.], 2009.

KJELLGREN, P. A semi-implicit fractional step finite element method for viscous incompressible flows. Computational Mechanics, v. 20, p. 541-550, 1997. 
KOOBUS, B.; FARHAT, C. Second-order time-accurate and geometrically conservative implicit schemes for flow computations on unstructured dynamic meshes. Comput. Methods Appl. Mech. Engrg., v. 170, p. 103-129, 1999.

KUHL, D.; RAMM, E. Generalized energy-momentum method for non-linear adaptative shell dynamics. Comput. Methods Appl. Mech. Engrg., v. 178, p. 343-366, 1999.

LANCZOS, C. The Variational Principles of Mechanics. 4. ed. New York: Dover, 1986.

LAUNDER, B. E.; SPALDING, D. B. Lectures in mathematical models of turbulence. New York: Academic Press, 1972.

LESOINNE, M.; FARHAT, C. Geometric conservation laws for flow problems with moving boundaries and deformable meshes, and their impact on aeroelastic computations. Comput. Methods Appl. Mech Engrg., v. 134, p. 71-90, 1996.

LI, B. Q. Discontinuous Finite Elements in Fluid Dynamics and Heat Transfer. London: Springer, 2006.

MALVERN, L. E. Introduction to the mechanics of a continuous medium. New Jersey: Prentice-Hall, 1969.

MARTIN, J. C.; MOYCE, W. J. An experimental study of the collapse of liquid columns on a rigid horizontal plane. Philosophical Transactions of the Royal Society of London. Series A, v. 244, p. 312-324, 1952.

MELCHIOR, S. A.; LEGAT, V.; DOOREN, P. V.; WATHEN, A. J. Analysis of preconditioned iterative solvers for incompressible flow problems. Int. J. Numer. Meth. Fluids, v. 68, p. 269-286, 2012.

MOK, D. P. Partitionierte Lösungsansätze in der Strukturdynamik und der Fluid-StrukturInteraktion. Tese (Doutorado) - Institut für Baustatik der Universität Stuttgart, Stuttgart, 2001.

MORTON, S. A.; MELVILLE, R. B.; VISBAL, M. R. Accuracy and coupling issues of aeroelastic navier-stokes solutions on deforming meshes. Journal of Aircraft, v. 35, p. 798-805, 1998.

NAJAFI, M.; AREFMANESH, A.; ENJILELA, V. Meshless local petrov-galerkin method-higher reynolds numbers fluid flow applications. Engineering Analysis with Boundary Elements, v. 36, p. $1671-1685,2012$.

NIGRO, N.; STORTI, M.; IDELSOHN, S.; TEZDUYAR, T. Physics based gmres preconditioner for compressible and incompressible navier-stokes equations. Comput. Methods Appl. Mech. Engrg., v. 154, p. 203-228, 1998.

NITHIARASU, P. An arbitrary lagrangian eulerian (ale) formulation for free surface flows using the characteristic-based split (cbs) scheme. Int. J. Numer. Meth. Fluids, v. 48, p. 1415-1428, 2005.

NITHIARASU, P.; LIU, C. B. An artificial compressibility based characteristic based split (cbs) scheme for steady and unsteady turbulent incompressible flows. Comput. Methods Appl. Mech. Engrg., v. 195, p. 2961-2982, 2006. 
NOBILE, F.; VERGARA, C. An effective fluid-structure interaction formulation for vascular dynamics by generalized robin conditions. SIAM Journal on Scientific Computing, v. 30, n. 2, p. 731-763, 2008.

OGDEN, R. W. Non-linear elastic deformations. Chichester, England.: Ellis Harwood, 1984.

PIPERNO, S. Explicit-implicit fluid-structure staggered procedures with a structural predictor and fluid subcycling for $2 \mathrm{~d}$ inviscid aeroelastic simulations. Int. J. Numer. Meth. Fluids, v. 25, p. 1207-1226, 1997.

POTTER, M. C.; WIGGERT, D. C. Mecânica dos Fluidos. 3. ed. São Paulo: Cengage Learning, 2009.

REDDY, J. N.; GARTLING, D. K. The Finite Element Method in Heat Transfer and Fluid Dynamics. 3. ed. Boca Raton, FL: CRC Press, 2010.

ROUX, F. X.; GARAUD, J. D. Domain decomposition methodology with robin interface matching conditions for solving strongly coupled fluid-structure problems. International Journal for Multiscale Computational Engineering, v. 7, p. 29-38, 2009.

SAMPAIO, P. A. B. de; LYRA, P. R. M.; MORGAN, K.; WEATHERILL, N. P. Petrov-galerkin solutions of the incompressible navier-stokes equations in primitive variables with adaptative remeshing. Comput. Methods Appl. Mech. Engrg., v. 106, p. 143-178, 1993.

SANCHES, R. A. K. Sobre o acoplamento fluido-casca utilizando o método dos elementos finitos. Tese (Doutorado) — SET-EESC-USP, São Carlos, São Paulo, Brasil, 2011.

SANCHES, R. A. K.; CODA, H. B. An embedded domain technique based on level-sets for finite element method (fem) fluid-shell coupling. Mecánica Computacional, XXIX, p. 4801-4818, 2010a.

SANCHES, R. A. K.; CODA, H. B. Fluid-structure interaction using an arbitrary lagrangianeulerian fluid solver coupled to a positional lagrangian shell solver. Mecánica Computacional, XXIX, p. 1627-1647, 2010b.

SANCHES, R. A. K.; CODA, H. B. Unconstrained vector nonlinear dynamic shell formulation applied to fluid-structure interaction. Computer Methods in Applied Mechanics and Engeneering, v. 259 , p. $177-196,2013$.

SANCHES, R. A. K.; CODA, H. B. On fluid-shell coupling using an arbitrary lagrangianeulerian fluid solver coupled to a positional lagrangian shell solver. Applied Mathematical Modelling, v. 38, p. 3401-3418, 2014.

SIMO, J. C.; FOX, D. D. On a stress resultant geometrically exact shell model. part i: formulation and optimal parametrization. Comput. Methods Appl. Mech. Engrg., v. 72, p. 267-304, 1989.

STRANG, G.; FIX, G. An analysis of the Finite Element Method. 2. ed. [S.1.]: Wesley-Cambridge Press, 2008.

TALLEC, P. L.; MOURO, J. Fluid structure interaction with large structural displacements. Comput. Methods Appl. Mech. Engrg., v. 190, p. 3039-3067, 2001. 
TEIXEIRA, P. R. F. E.; AWRUCH, A. M. Numerical simulation of fluid-structure interaction using the finite element method. Computers and Fluids, v. 34, p. 249-273, 2005.

TEZDUYAR, T. E.; MITTAL, S.; RAY, S. E.; SHIH, R. Incompressible flow computations with stabilized bilinear and linear equal-order-interpolation velocity-pressure elements. Comput. Methods Appl. Mech. Engrg., v. 95, p. 221-242, 1992.

TEZDUYAR, T. E.; SENGA, M. Stabilization and shock-capturing parameters in supg formulation of compressible flows. Computer Methods in Applied Mechanics and Engineering, v. 195, n. 13-16, p. 1621-1632, 2006.

THOMAS, C. G.; NITHIARASU, P.; BEVAN, R. L. T. The locally conservative galerkin (lcg) method for solving the incompressible navier-stokes equations. International Journal for Numerical Methods in Fluids, v. 57, p. 1771-1792, 2008.

THOMAS, P. D.; LOMBARD, C. H. Geometric conservation law and its applications to flow computations on moving grids. AIAA Journal, v. 17, p. 1030-1037, 1979.

TRUESDELL, C. A. Hypo-elasticity. J. Rational Mech. Anal., v. 4, p. 83-133, 1955.

VÁZQUEZ, J. G. V. Nonlinear analysis of orthotropic membrane and shell structures including fluid-structure interaction. Tese (Doutorado) - Universitat Politecnica de Catalunya, Barcelona, Espanha, 2007.

WALL, W. A.; RAMM, E. Fluid structure interaction based upon a stabilized (ale) finite element method. In: IDELSHON, S. et al. (Ed.). Computational Mechanics. Barcelona: CIMNE, 1998.

WARBURTON, G. B. The Dynamical Behaviour of Structures. 2. ed. Oxford: Pergamon Press, 1976.

WILCOX, D. C. Turbulence modeling for CFD. La Cañada, CA: DCW Industries Inc., 1993.

ZIENKIEWICZ, O. C.; TAYLOR, R. L. The Finite Element Method: The Basis. 6. ed. [S.1.]: Butterworth Heinemann Linacre house, 2000. v. 1. 689 p.

ZIENKIEWICZ, O. C.; TAYLOR, R. L.; NITHIARASU, P. The Finite Element Method: Fluid Dynamics. 6. ed. [S.1.]: Butterworth Heinemann Linacre house, 2005. v. 3.334 p.

ZIENKIEWICZ, O. C.; TAYLOR, R. L.; SHERWIN, S. J.; PEIRÓ, J. On discontinuous galerkin methods. Int. J. Numer. Engng., v. 58, p. 1119-1148, 2003. 